\title{
REDUCED GLUTATHIONE AND CYSTEINE HYDROCHLORIDE ON CROSSBRED BULL SEMEN
}

\section{PERUMAL P.}

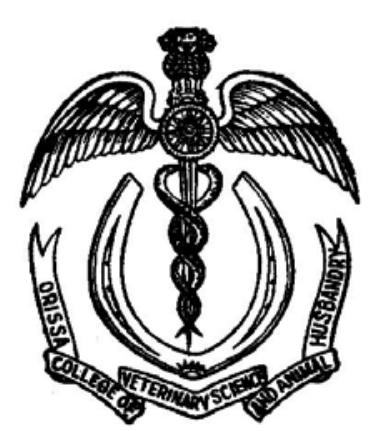

DEPARTMENT OF GYNAECOLOGY AND OBSTETRICS FACULTY OF VETERINARY SCIENCE AND ANIMAL HUSBANDRY ORISSA UNIVERSITY OF AGRICULTURE AND TECHNOLOGY BHUBANESWAR-751003, India 


$$
\begin{gathered}
\text { Dedicated } \\
\text { To } \\
\text { My beloved Parents } \\
\text { My beloved God Lord Sannasi Swamy } \\
\text { And } \\
\text { My beloved Teachers }
\end{gathered}
$$




\begin{tabular}{llc} 
I & INTRODUCTION & 1 \\
II & REVIEW OF LITERATURE & 4 \\
III & MATERIALS AND METHODS & 36 \\
IV & RESULTS & 48 \\
V & DISCUSSION & 84 \\
VI & SUMMARY & 101 \\
VII & CONCLUSION & 105 \\
& BIBLIOGRAPHY & 106 \\
\hline
\end{tabular}

\section{LIST OF TABLES}


1 Seminal attributes of Good and Poor freezable crossbred bulls

2 Post thaw Sperm Motility, Livability and Total abnormal sperm percent of Good and Poor freezable Crossbred bulls in different diluents

Post thaw Sperm Loss of Acrosomal Integrity, HypoOsmotic Swollen positive sperms and Cervical Mucus Penetration rate of Good and Poor freezable Crossbred bulls in different diluents

$4 \quad$ Post thaw Sperm DNA fragmentation (percent), Lipid Peroxidation (nmol/108 sperms) and percent of Mitochondrial Membrane Potential of Good and Poor freezable crossbred bulls in different diluents Assessment of Motility at different hours of incubation in Good and Poor freezable Crossbred bull semen (CASA)

6 Assessment of Motility At different Hours of incubation in Good And Poor freezable Crossbred bull Semen (CASA) in different experimental groups

7 Computer Assisted Semen Analysis (CASA) of some post-thaw seminal characteristics in control and treatment groups at different hours of incubation

8 Conception rate of crossbred Jersey bulls with different semen additives

9. and conception rate in crossbred jersey bulls 
1. Post thaw Sperm Motility of semen of good and poor freezable Crossbred Jersey bulls in different diluents

2. Post thaw Sperm livability of semen of good and poor freezable Crossbred Jersey bulls in different diluents

3. Post thaw Total Sperm Abnormality of semen of good and poor freezable Crossbred Jersey bulls

4. Post thaw loss of acrosomal integrity of semen of good and poor freezable Crossbred Jersey bulls

5. Post thaw HOST of semen of good and poor freezable Crossbred Jersey bulls

6. Post thaw CMPT of sperm of semen of good and poor freezable Crossbred Jersey bulls

7. Acrosomal Integrity (FITC-PSA)

8. Hypo osmotic swelling test

9. Post thaw DNA fragmentation of sperm of semen of good and poor freezable Crossbred Jersey bulls

10. Post thaw MMP of sperm of semen of good and poor freezable Crossbred Jersey bulls

11. Post thaw LPO of sperm of semen of good and poor freezable Crossbred Jersey bulls

12. DNA fragmentation (nuclear integrity) by Fuelgen's staining

13. Mitochondrial Membrane Potential ( JC- 1 Staining)

14. Post thaw seminal attributes of good and poor freezable Crossbred Jersey bulls

15. Mobility parameters of control, cysteine and glutathione added semen of good freezable Crossbred Jersey bulls

16. Mobility parameters of control, cysteine and glutathione added semen of poor freezable Crossbred Jersey bulls

17. Computer assisted sperm analysis of post thaw semen

18. Computer Assisted Semen Analysis of post-thaw seminal motility parameters

19. CASA Parameters of good and poor freezable crossbred Jersey bulls

20. CASA Parameters of control, cysteine and glutathione added semen of good crossbred Jersey bulls 
21. CASA Parameters of control, cysteine and glutathione added semen poor freezable Crossbred Jersey bulls

22. Post insemination conception rate of crossbred Jersey bulls with different semen additives 


\section{LIST OF ABBREVIATIONS}

$\Delta \psi \mathrm{m} \quad: \quad$ Mitochondrial Membrane Potential

$\mu \mathrm{M} \quad$ : Micro mole

AI $\quad$ : Artificial Insemination

AR : Analytical Grade

ATP : Adenosine Triphosphate

AV : Artificial Vagina

BCF : Beat/Cross Frequency

BCM : Bovine Cervical Mucus

BSA : Bovine Serum Albumin

CASA : Comport Assisted Sperm Analysis

CB : Crossbred

CFDA : 6-carboxyfluorescein diacetate

CMPT : Cervical Mucus Penetration Test

ConA : Concanavalia ensiformis

DPBS : Dulbecco's modified Phosphate Buffered Saline

EYCG : Egg Yolk Glycerol

EYTG : Egg Yolk Tris Glycerol

FITC-PSA : Fluorescein isothiocyanate-conjugated Pissum sativum agglutinin

FITC-RCA : Fluorescein isothiocyanate-conjugated Ricinus Communis Agglutinin

GPX : Glutathione Peroxidase

GSH : Reduced Glutathione

GSSG : Glutathione

HMMP $\quad$ : High Mitochondrial Membrane Potential

HOST : Hypo-Osmotic Swelling Test

IVF $\quad$ : In - vitro fertilization

$\mathrm{JC}-1$

: $\quad$ 5,5',6,6'-tetrachloro-1,1',3,3'-tetraethylbenzimidazolyl carbocyanine

LIN : Linearity

MDA : Malondialdehyde

$\mathrm{mM} \quad:$ millimole 


$\begin{array}{lll}\text { MMP } & : \text { Mitochondrial Membrane Potential } \\ \text { mOsm } & : \text { Milliosmole } \\ \text { NAC } & : \text { N-acetyl-1-cysteine } \\ \text { NRR } & : \text { Non-return rate } \\ \text { PAG } & : \text { Poly Acrolamide gel } \\ \text { PBS } & : \text { Phosphate buffered saline } \\ \text { PI } & : \text { Propidium iodide } \\ \text { PNA } & : \text { Arachis hypogea agglutinin } \\ \text { PUFA } & : \text { Poly Unsaturated Fatty Acid } \\ \text { RFU } & : \text { Relative fluorescence units } \\ \text { ROS } & : \text { Reactive Oxygen Species } \\ \text { SH } & : \text { Sulfa hydryl group } \\ \text { SMPT } & : \text { Sc } & \text { Sperm-mucus penetration test (sperm concentration) } \\ \text { SMPT } & : \text { sd } & \text { sperm-mucus penetration test (vanguard distance) } \\ \text { SOD } & : \text { Super Oxide Dismutase } \\ \text { SPD } & : \text { Sperm Penetration Distance } \\ \text { SPS } & : \text { Sperm penetration speed } \\ \text { STR } & : \text { Straightness } \\ \text { TBA } & : \text { Thiobarbituric acid } \\ \text { TCA } & : \text { Tri-chloral acetic acid } \\ \text { TYG } & : \text { Tris Yolk Glycerol } \\ \text { VAP } & : \text { Average path Velocity } \\ \text { VCL } & : \text { Curvilinear Velocity } \\ \text { VLH } & : \text { Amplitude of Lateral Head Displacement } \\ \text { VSL } & : \text { Straight line Velocity } \\ \text { WOB } & : \text { Wobble } \\ \text { ZP } & : \text { Zona pellucida } \\ & & \end{array}$




\section{ACKNOWLEDGEMENT}

Words fail to express my deepest sense of gratitude and indebtedness to my guide and esteemed Dr. A.K. Barik, Associate Professor, Department of Gynaecology and Obstetrics, Faculty of Veterinary Science and Animal Husbandry, Bhubaneswar for his able guidance, constant supervision, relentless efforts, constructive counsel and affectionate attitude during the entire course of my investigation and preparation of this manuscript.

I express my hearty regards, deep sense of gratitude to Dr. D. N. Mohanty, Ph.D., Associate Professor and Head, Department of Animal Reproduction, Gynaecology and Obstetrics, College of Veterinary Science and Animal Husbandry, Bhubaneswar for conceptualization of this experiment, scholastic guidance, constant inspiration and supervision, sustained interest, constructive criticism and good effort throughout my course of study.

My special obligations are due to Dr. R.K Das, Professor and Head Department of Animal anatomy and Histology for his moral support, prudent suggestions, timely encouragement and providing necessary facilities during the course of investigation.

I am extremely grateful to Dr. S.Selvaraju, Scientist (SS), Reproductive physiology division, National institute of animal nutrition and physiology, Bangalore for his inspiration, valuable suggestions and providing necessary facilities during the course of investigation.

Word fails to express my sincere sense of reverence to Dr. S.K.H. Ray, Ex-Head, Department of Gynaecology and Obstetrics, Orissa College of Veterinary Science and Animal Husbandry, Bhubaneswar for his sincere interest, good guidance throughout the course of my study.

I am proud of expressing my sincere gratitude to Dr. Srinibas Das, Director, Teaching Veterinary Clinical Complex for his sincere interest, sustained guidance and painstaking effort throughout the course of my study.

I acknowledge gladly the assistance rendered by Dr. P.C. Mishra, Assistant Professor, Department of Animal Reproduction, Gynaecology and Obstetrics, for his sincere and painstaking efforts and moral, financial and technical support and motivation throughout the course of my investigation.

I am obliged to the Director of Animal Husbandry \& Veterinary Services, Government of Orissa, Cuttack for giving me permission to conduct experiment in collaboration with the Frozen Semen Bank, Khapuria, Cuttack. 
I express my profound sense of gratitude to Dr. J.K.Pradhan, Deputy Director, Frozen Semen Bank, Cuttack for providing me necessary facilities during the course of investigation.

I am very grateful to Dr. H.B. Nayak, Assistant Director, Frozen Semen Bank (Laboratory), Cuttack for his able suggestions and providing the necessary facilities in course of semen evaluation in the semen laboratory.

I am extremely grateful to Dr. R.M. Moharatha, Assistant Director, Frozen Semen Bank, Cuttack for his constant inspiration, scholastic guidance and painstaking efforts throughout the period of experiments.

I am honestly grateful to Dr. Rashmi Ranjan Dash, Research Assistant, Frozen Semen Bank, Cuttack for his kind supervision and suggestions during the course of experiments.

I am grateful to Dr. B.N. Sahoo, Veterinary Assistant Surgeon, Frozen Semen Bank, Cuttack for his kind cooperation during course of semen collection.

I acknowledge gladly the assistance rendered by Dr. Prasanta Das, Quality Control Officer Dr. S.K. Pradhan, Microbiologist, Frozen Semen Bank, Cuttack for their sincere support during the course of semen processing.

I am grateful to Dr. Mahendra Ray, Veterinary Assistant Surgeon, Veterinary poly clinic, Sahid Nagar, Bhubaneswar for his kind cooperation in field fertility trials during the course of experimentation.

I am grateful to Dr. Sunil Das, Veterinary Assistant Surgeon, Veterinary Dispensary, Nayapalli, Bhubaneswar for his kind cooperation in field fertility trials during the course of experimentation.

I am grateful to Dr. K.C.Das, SRF, DBT RBC project, OUAT for his kind cooperation in field fertility trials during the course of experimentation.

I am very grateful to the Branch Manager, Canara bank, Viruveedu village, Dindigul Dist, Tamilnadu for providing the financial support in the form of education loan throughout course of study without any hesitation.

My special thanks to laboratory assistants and technicians of FSB, Cuttack for their assistance during the course of semen evaluation, processing, preservation and to Livestock Inspector and attendants for their cooperation during the course of semen collection, cleaning and sterilization. 
I am privileged to express my sincere thanks to my post graduate colleagues namely Dr. N.C.Pattanaik, Dr. J.K.Chand, Juniors Dr. Debaraj Nayak and Dr. P.K. Swain, MCA friends namely Satya Narayana Sahu, Susantha Kumar Behare, Biresh Kerkata, Babulu Behera, and Dr.Siva Subramanian, Research associate, Mr. Ragavendra, Senior Research fellow, Mr. Selvakumar, Technical assistant of Reproductive physiology division, National institute of animal nutrition and physiology, Bangalore and other post graduate colleagues like Dr. Manimaran, Dr. Mohan Das and Dr. Chandran for their good will and moral support during the course of the study.

I am thankful to "Raja Communication" D.T.P. Centre, Gopabandhu Square, Unit-8, Bhubaneswar for timely typing the manuscript.

I express my sincere thanks to Mr. Mulia Babu, Livestock Inspector, Veterinary poly clinic Sahid Nagar, Bhubaneswar, Mr. G.C.S. Achary, Technician and other Departmental sub-staff namely Sridhar, Jagadish, Nityananda, Sakuntala, Central clinic sub-staff Mr. Kelu Biswal and Mr. Sanjeeve Kumar Das laboratory attendant for their kind cooperation and timely help during the course of my investigation.

Words fail to express my intrinsic gratitude to my brothers Mr. P. Chandrasekaran, Mr. P. Sasikumar, Sister Mrs. S. Rajeshwari, Brother-in-law Mr.A.Saravanan, Sister-in-laws Mrs. C. Nandhini, Mrs. S.Kavitha, my dear Kutty Chellum RaviRajan and my beloved parents whose gracious sacrifice and encouragement despite silent suffering of immeasurable magnitude, helped me to complete this investigation.

(PERUMAL P.) 


\section{INTRODUCTION}

Artificial insemination is the first generation biotechnological advancement that has made a profound contribution to the genetic improvement, particularly in dairy bulls through which a single ejaculate from male is used to impregnate many females. This impact could not have been possible without long standing freezing of bull semen. The principle of cryopreservation is a state of suspended animation, which regains its capability of fertilization after processing that further, adds an advantageous role in adopting the technology. Long term storage facilitates semen transport over distances and enables extended use of superior germplasm, even after the sire's death. This has attained a status of industry especially for the dairy animals due to wide use of frozen semen, but as yet semen cryopreservation could not attain status of reality because of the fact that following freezing and thawing a large number of sperms are rendered incapable to fertilize the ovum. Various steps of cryopreservation such as processing, freezing and thawing exert physiological as well as chemical stress on the sperm membrane (Chatterjee et al., 2001), associated with an oxidative stress induced by free radical (Salvader et al.,2006). Sperm cells have a high content of unsaturated fatty acids in their membrane but lack in a significant cytoplasmic component containing antioxidants. Hence, they are highly susceptible to lipid peroxidation by oxygen free radicals and $\mathrm{H}_{2} \mathrm{O}_{2}$ (Sinha et al., 1996). So, a variety of additives like anti-oxidants, membrane stabilizers, motility enhancers and chelating agents have been used to protect spermatozoa from deleterious effects of cryopreservation and for improving freezability and fertility of bull semen.

Oxidative damage to sperm resulting from reactive oxygen species, generated by the cellular components of semen during liquid storage, is possibly one of the main causes for the decline in motility and fertility during storage, while the other detrimental cause is low temperature on the destabilization of sperm membrane structure (Mustafa and Necmettin, 2007). At present, it is generally accepted that the consequences of sperm cryo-injury caused by the cryopreservation procedure are impaired transport and poor survival in the female reproductive tract (Salamon and Maxwell, 1995).

The major disadvantage of cryopreservation is death of the half of the sperm population during freezing and thawing procedure even if most adoptive preservation technique is followed. In addition to it, changes in temperature impart changes on the composition and structure of sperm plasma membrane integrity by adversely modifying their function (Krogenaes et al., 1994).

Spermatozoa are the terminal cell following a complex developmental process. The semen evaluation is an ideal method to judge indirectly, the reproductive efficiency of a bull. 
Routine semen analysis is an important criterion to assess fertility of a bull. Various seminal attributes like volume, mass activity, individual motility, sperm concentration and sperm abnormalities can be assessed with minimum infrastructural facilities both in chilled and frozen semen. Each of these parameters has direct or indirect bearing potency of a breeding bull. A minimum standard for each aspect of semen evaluation has to be followed for successful breeding. So semen evaluation is the basic need for andrological investigation of breeding bulls. Normal sperm counts and other variables measured in routine semen analysis, do not comprehensively ensure fertility. Other factors, not measured in routine semen analysis, such as cytokines and reactive oxygen species have been related to infertility (Iwasaki and Gagnon, 1992; Huszar and Vigue, 1994 and Sharma and Agarwal, 1994).

The present study aims at improving the post thaw recovery of spermatozoa after freeze thaw cycle, consequent to addition of cysteine hydrochloride and reduced glutathione to the conventional extender (EYTG) to minimize the risk factors involved in ultra low freezing process. Hydrogen peroxide is the product of oxidative de-amination of aromatic amino acids. It accumulates in appreciable quantity in semen sample. This is detrimental to sperm survival and that can be neutralized by addition of anti-oxidants. Besides this, the implication of such application will be assessed on the basis of resultant conception rate from fertility trial. In the light of above facts, the experiment was designed with the objectives of to study the effect of Cysteine hydrochloride and reduced glutathione on seminal attributes during the process of freezing and thawing in good freezable and poor freezable bull semen, to evaluate sperm membrane integrity and viability of sperm before and after cryopreservation by computer assisted semen analysis, to undertake certain in vitro tests like HOST and CMPT of semen after addition of semen additives in cryopreserved semen and to analyze the effect of additives in good freezable and poor freezable bull semen on the basis of conception rate following field fertility trials. 
In the light of above facts, the experiment was designed with following objectives.

- To study the effect of Cysteine and Glutathione on seminal attributes during the process of freezing and thawing in good freezable and poor freezable bull semen.

- To evaluate sperm membrane integrity and viability of sperm before and after cryopreservation by computer assisted semen analysis.

- To undertake certain in vitro tests like HOST and CMPT of semen after addition of semen additives in cryopreserved semen.

- To analyze the effect of additives in good freezable and poor freezable bull semen on the basis of conception rate following field fertility trials. 


\section{REVIEW OF LITERATURE}

Improving the quality of frozen semen, minimizing the post thaw discard rate and increasing conception rate have been tried by several workers by using different additives like enzymes, proteins, amino acids, anti-oxidants, carbohydrates, hormones, vitamins etc. The present experiment dealt with addition of cysteine and glutathione to the normal EYTG semen extender. Relevant literature and corroborate reports from India and abroad have been reviewed.

\section{ROUTINE SEMEN EVALUATION}

\section{Volume}

It is inferred that an increase or decrease in volume of semen is not usually correlated with fertility or sterility in a male unless ejaculation fails to occur (Roberts, 1971).

The ejaculated volume significantly increased with age (Abdel-Raouf, 1965; Colchen-Bourlaoud, 1972 and Rao and Rao, 1979) and the same was increased linearly with age along with motile spermatozoa and sperm concentration, which was correlated with total sperm count (Almquist and Cunningham, 1967; Colchen-Bourlaoud and Thibier, 1973). A correlation between total no. of sperm and semen volume was also reported by Nishiyama et al. (1968a).

The over all mean seminal volume(ml), mass activity (0-4), individual motility (\%), live sperm (\%), sperm concentration (million/ml), normal sperm (\%) and conception rate (\%) are $2.42 \pm$ $0.22,2.77 \pm 0.09,57.48 \pm 1.83,86.51 \pm 0.81,1316 \pm 44,87.30 \pm 1.64$ and $35.53 \pm 2.16$ respectively in Crossbred Jersey bulls (Bedi et al.,1984).

The average volume for $62.5 \% \mathrm{JY}$ bulls was significantly higher than other genetic groups except 75\% HF bulls (Sagdeo et al., 1990).

The mean ejaculated volume (double thrust) recorded in Gir bulls $(7.03 \pm 0.44 \mathrm{ml})$ did not differ significantly from that in Jaffarabady bulls $(6.36 \pm 0.33 \mathrm{ml})$ as reported by Raina and Dhami (2004).

Volume of Friesian Gir cross bred (5.278 \pm 0.056$)$ was significantly higher than JY Friesian cross bred (4.473 \pm 0.083$)$ as reported by Bhoite et al., (2008).

Volume $(\mathrm{ml})$, colour, concentration $\left(\times 10^{6} / \mathrm{ml}\right)$, mass activity, motility $(\%)$, normal acrosome, mid-piece, tail (\%) and normal head (\%) were $4.5 \pm 0.4,3.5 \pm 0.3,1410.0 \pm 70.0,3.8 \pm 0.3,77.5 \pm$ 1.5, $93.5 \pm 0.5$ and $93.5 \pm 1.0$ respectively in fresh semen of HF Crossbred bull (Munsi et al.,2007) 
Ejaculate volume $(\mathrm{ml})$, sperm motility $(\%)$, concentration $\left(\mathrm{X} 10^{6} / \mathrm{ml}\right)$, total abnormality $(\%)$, total viability, HOST and $\mathrm{pH}$ were $5.03 \pm 0.2,83.5 \pm 2.4,1480.0 \pm 70.0,5.40 \pm 0.6,82.30 \pm 2.8$, $83.80 \pm 3.3$ and $6.59 \pm 0.0$ respectively in fresh semen of bull (Uysal et al.,2007).

\section{Colour}

The colour of semen was reported to be dependent upon the sperm concentration of the ejaculate (Tripathy and Prabhu, 1968 and ZemJanis, 1970).

The normal colour of bull semen is milky white or light yellow (Roberts, 1971). However, ZemJanis (1970) mentioned the colour of semen and consistency as creamy, milky-opaque, opalescent and watery with densities 3,2,1 and 0, containing more than 10,00,000; more than $5,00,000-10,00,000 ; 2,00,000-5,00,000$ and less than 2,00,000 spermatozoa per $\mathrm{mm}^{3}$ of semen respectively.

It has been suggested that semen samples with abnormal colour and consistency should be examined for determining actual concentration of spermatozoa, as reduced concentration leads to low fertility (Roberts, 1971).

Ejaculates of reddish, brownish and blackish colour could be due to bleeding of capillaries on the surface of the glans, bleeding into seminal vesicle and tumour respectively (Bhosrekar, 1990).

The over all mean colour pattern of semen in two and three based crosses were 85.55 percent (creamy), 2.88 percent (yellow) and 11.57 percent (white).These results inferred that in all cross bred bulls the creamy colour of semen was dominant followed by white and yellow (Bhoite et al., 2008).

\section{Mass Activity}

Mass activity is evaluated as a routine test carried out to assess semen quality done immediately after collection. Wave motion is the out come of oriented sperm cell movement, which come into phases and it can be observed only in high density specimens such as ram or bull semen, but hardly in man, rabbit or other animals, whose semen is less dense (Mann and Lutwak-Mann, 1981).

Motility below 50\% was often associated with low conception rates and poor fertility (Blom, 1950). The initial motility was more closely related with semen characteristics and fertility (Bishop et al., 1954 and Erb et al., 1956). 
Gross motility of spermatozoa reflects the combined effect of sperm cell concentration and the viability of sperm cells (ZemJanis, 1970). Mass activity is most subjective test and can vary from individual to individual (Bhosrekar, 1990).

Average mass activity of Pure JY, 75\% JY, 62.5\% JY,50\% JY, 75\%HF and 62.5\% HF was $3.04 \pm 0.03,2.07 \pm 0.12,2.30 \pm 0.15,1.93 \pm 0.06,1.77 \pm 0.19$ and $2.27 \pm 0.08$ respectively and $50 \%$ and $75 \%$ JY bulls have showed similar values. Jersey and HF bulls of $62.5 \%$ blood are also similar in this respect but were slightly superior to $75 \%$ HF bulls (Sagdeo et al., 1990).

The mean mass activity score and percent individual sperm motility were significantly higher $(\mathrm{p}<0.05)$ in Gir bulls $(3.33 \pm 0.11$ and $7.50 \pm 0.89)$ than Jaffarabady buffalo semen $(2.80$ \pm 0.06 and $66.75 \pm 1.04)$ as reported by Raina and Dhami (2004).

\section{Individual Motility}

Bovine semen should contain a minimum of 40 to $50 \%$ motile sperm, as low motility was associated with infertility (Blom, 1950). While, Branton et al., (1951) opined a minimum initial sperm motility of $70 \%$ required for efficient breeding policy. Although the motility of fertile bulls was higher than infertile bulls, the decrease in motility did not parallel to the decrease in fertility (Rollinson, 1951).

Blom (1950) and Bishop et al. (1954) reported that the initial motility has a positive corelation with sperm concentration and fertility, where as Lasley (1951) found a highly significant co-relation among percentage of live motile and progressively motile spermatozoa in fresh semen.

Abdel-Raouf (1965) and Almquist and Cunnigham (1967) found an increase in percentage of motile spermatozoa with advancement of age. However, the effect of season on initial motility was demonstrated by Tripathy and Prabhu (1968), who could not found significant effect of season. But Dojcseva et al. (1979) reported significant decrease in motile sperm.

The average motility of pure bred and cross bred bull was $82.84 \pm 0.77$ and $75.26 \pm 2.57 \%$ respectively. Live sperm and sperm concentration (millions $/ \mathrm{ml}$ ) were $84.37 \pm 0.81,73.63 \pm 2.5 \%$, $1003.92 \pm 50.79$ and $1176.31 \pm 16.13$ respectively (Barik et al., 1987).

The initial motility of Pure JY, 75\% JY, 62.5\% JY, 62.5\% HF and 75\% HF were $72.14 \pm$ $0.65 \%, 47.50 \pm 4.62 \%, 52.07 \pm 1.77 \%, 46.69 \pm 2.23 \%$ and $30.24 \pm 2.24 \%$ respectively (Sagdeo et al., 1990). 
Satisfactory bulls have the scrotal circumference, motility (\%), concentration of sperm $\left(\mathrm{X} 10^{6} / \mathrm{ml}\right)$ and live sperm \% were $34.56 \pm 2.85,82.09 \pm 2.15,1220.83 \pm 99.26,87.27 \pm 2.42$ and corresponding value for unsatisfactory bulls were $27.75 \pm 1.85,58.83 \pm 20.18,831.48 \pm 1.85$ and $65.76 \pm 21.80$ respectively (Rao and Rao,1979).

Mean value for initial and pre-freeze motility were significantly higher $(\mathrm{p}<0.05)$ in cattle $(81.67 \pm 1.28$ and $72.87 \pm 1.47)$ than in buffalo $(76.94 \pm 1.81 \%$ and $67.76 \pm 2.03 \%)$. Semen post thaw motility also did not vary between them $(45.23 \pm 1.71$ Vs $44.49 \pm 1.99 \%)$ as reported by Dhami et al. (1991).

The initial motility in 75\% cross bred bull was $67.77 \%$ (Chauhan et al., 1993). Good initial motility alone is not an accurate indicator of fertility in bulls (Swanson and Herman, 1994)

The velocity of sperm is an indirect measure of the force of movement. Penetration of cumulous oophorus does not depend upon the degree of progressiveness of movement but it does require reasonable velocity. A small reduction in velocity may affect sperm penetration of zona pellucida (Olds - Clarke, 1996).

Baumber et al. (2000) had reported that sperm motility may be a more sensitive indicator of oxidative stress and is therefore inhibited before any measurable increase in lipid peroxidation and the sperm motility may be affected by ROS through a mechanism of action separate to that of lipid peroxidation. In somatic cell systems, it has been proposed that $\mathrm{H}_{2} \mathrm{O}_{2}$ causes perturbations in important biochemical functions, including increased formation of oxidized intracellular sulfahydryls, rapid decrease in ATP levels, and a consequent depression of glycolytic flux.

\section{Sperm Concentration}

Lagerlof (1934) reported that the sperm concentration in fertile bulls varied from $30 \times 10^{6}$ to $200 \times 10^{6}$ per $\mathrm{mm}^{3}$.

Haq (1949) found that spermatozoan density remained within normal limits in many bulls with testicular degeneration. However, in infertile or sterile bulls, there was a rapid decline on sperm concentration among first, second and third ejaculates indicting a poor reserve with reduced sperm production (Rollinson, 1951).

Branton et al. (1951) reported that sperm concentration of at least 900 million per $\mathrm{mm}^{3}$ is required for efficient breeding. 
A proportionate increase in sperm concentration with advancement of age was reported by Colchen - Bourlaud and Thibier (1972) and Rao and Rao (1979). On contrary, Schluter (1975) observed no significant effect of age on sperm concentration.

Nishiyama et al. (1968 a) found a co-relation between sperm concentration and environmental temperature. Similarly Porwel et al. (1977) reported highly significant effect of season, while Dojcseva et al. (1979) described a decrease in sperm concentration during winter season.

Sperm concentration per $\mathrm{ml}$ is one of the most important traits of semen quality for acceptance in frozen semen production. The average sperm concentration in Gir and Jaffarabady bull semen was $2608 \pm 53$ and $1384 \pm 40$ millions/ml, respectively (Raina and Dhami, 2004).

\section{Live Spermatozoa Count}

A different staining method for differentiating live and dead bull spermatozoa was proposed by Blom (1950) using eosin as the sperm staining substance and nigrosin as the background stain. This mixture gave a very uniform preparation with stained (dead) cells clearly distinguishable from the live or unstained cells. The nigrosin provides a smoother background stain than fast green, opal blue, or aniline blue. It seems to obscure the sperm less and mixes with seminal plasma or diluter more uniformly. For this reason it can be used as easily with egg-yolk diluted -semen as with semen not mixed with egg yolk.

The proper staining of dead sperm was found by Mayer et al. (1951) to be dependant upon careful control of $\mathrm{pH}$, osmotic pressure, stain concentration and ionizable salts.

Bishop et al. (1954) described that variation in the metabolic activity of the semen cause variation in concentration of live spermatozoa and incidence of dead spermatozoa.

The proportions of live and dead spermatozoa are varying between ejaculates and bulls. Madden et al. (1947) observed that the fertility decline when the live sperm count was below 50\%. Moreover, semen with $30 \%$ of dead spermatozoa reduced fertility in cattle (Bonnadonna and Kann, 1955).

Colchen-Bourlaoud and Thibier (1973) observed that percentage of dead spermatozoa decreased with increase in age. Mohanty (1981) observed that increase in dead spermatozoa with advancement of age was attributed to impairment of epididymal function. 
The proportion of dead cells was $18 \%$ in the fresh semen and increased to $46 \%$ after freezing-thawing (Chen et al., 1989).

The mean percentages of live and dead sperms found in fresh semen of Gir bulls were 71.85 \pm 1.49 and $22.50 \pm 1.40$, respectively and the corresponding values in Jaffarabady buffalo bulls were $77.90 \pm 2.08$ and $22.30 \pm 1.57$. The mean live sperm percentage differed significantly between them (Raina and Dhami, 2004).

Overall mean value of semen volume $(\mathrm{ml})$, concentration $\left(\mathrm{X} 10^{6} / \mathrm{ml}\right)$, mass activity (0-5), initial motility (\%), live spermatozoa (\%), tail abnormality(\%), mid piece abnormality (\%), abnormal tail(\%) and total abnormal sperm (\%) were $3.15 \pm 0.13,1335.25 \pm 51.00,2.66 \pm 0.007$, $52.7 \pm 1.31,70.40 \pm 3.91,4.50 \pm 1.37,6.80 \pm 1.82,7.10 \pm 1.26$ and $18.40 \pm 3.03$ respectively in Sahiwal bulls (Mandal et al.,2005).

Mathur et al. (1991) who recorded the abnormality in semen of crossbred bull was $12.16 \%$. Singh et al. (1997) recorded overall sperm abnormality was $2.03 \%$ to $3.90 \%$ in crossbred semen.

Live spermatozoa $(\%)$ observed in IBFG $(78.58 \pm 0.288)$ was significantly higher than IFG $(76.59 \pm 0.14)$ and IJFG $(73.84 \pm 0.29)$ genetic groups. The live spermatozoa was highest in CB bulls having $50 \%$ Brown Swiss inheritance following Friesian and Jersey crosses (Bhoite et al., 2008)

\section{Diluent with Cysteine Hydrochloride}

Jonson et al. (1954) did not found any difference in livability of spermatozoa between samples treated with cysteine at the rate of $1 \mathrm{mg}$ per $\mathrm{ml}$ and controlled samples. Again, a similar result was obtained by addition of Glutathione. In another study they reported an increase in motility of spermatozoa in a heated skim milk containing cysteine hydrochloride.

Thiol compounds such as cysteine are precursors of intracellular Glutathione biosynthesis. Cysteine protects the sperm cells from toxic oxygen metabolites causing lipid peroxidation of the sperm plasma membrane under in-vitro condition (Meister and Tate, 1976).

Low molecular weight thiol compounds, such as cysteamine and p-mercaptoethanol reduce cysteine to cystine, promoting cysteine uptake in the cells and hence enhancing GSH synthesis (Bannai, 1984).

It is well known that GSH plays a critical role in protecting mammalian cells from oxidative damages. GSH synthesis is highly dependent on the availability of cysteine in the medium. This 
amino acid is very unstable in the extra cellular environment because it is rapidly oxidized to cystine. It is likely that the lack of cysteine in the medium, due to autoxidation to cystine, impairs GSH synthesis in vitro (Sagara et al., 1993).

Bilodeau et al. (2001) reported that the thiols such as glutathione and cysteine prevented the loss of sperm motility in frozen-thawed bull semen. Cysteine is a low molecular weight amino acid containing thiol and it is a precursor of intracellular glutathione biosynthesis, and increases the GSH level.

Post thaw sperm motility(\%), dead sperm (\%), abnormal acrosome (\%) and HOS test revealed that Cysteine $(5 \mathrm{mM})$ had more protective effect than taurine $(25 \mathrm{mM})$ and Trehalose $(50$ $\mathrm{mM}$ ) as additives in freezing of Sannen goat semen (Bucak and Uysal,2006).

Szcesniak-Fabianczk et al. (2006) reported that a semen extender with cysteine improved the viability, chromatin structure and membrane integrity of boar semen cells during liquid preservation.

It has been reported that the leakage of both AST and ALT enzymes was reduced by inclusion of EDTA and cysteine hydrochloride in the both the diluents as compared to control (EYC and TEYFC) extenders. It can be inferred that inclusion of cysteine hydrochloride and EDTA improves the keeping quality of buck semen (Singh et al., 2007).

Uysal et al. (2007) reported that many aspects of sperm protection like sperm motility, viability and membrane stabilization of sperm cells during relative cryopreservation are of prime importance. The antioxidants GSH $(5 \mathrm{mM})$ and Cysteine $(5 \mathrm{mM})$ provide a near optimal concentration for improved bovine sperm survival during the freezing -thawing process.

Post thaw sperm motility (\%), post thaw total abnormality (\%), post thaw acrosomal damage (\%), HOST (\%) and total viability (fluorescent staining) were 71.03 $\pm 3.1,10.8 \pm 1.0,8.9 \pm$ $0.8,79.3 \pm 3.7$ and $42.3 \pm 2.5 ; 44.0 \pm 3.1,11.0 \pm 1.5,9.1 \pm 1.1,51.3 \pm 3.3$ and $66.5 \pm 2.5 ; 53.0 \pm 3.2$ $, 17.8 \pm 0.9,10.0 \pm 1.3,59.2 \pm 3.4$ and $56.0 . \pm 3.0$ respectively for glutathione $(5 \mathrm{mM})$, cysteine $(5$ $\mathrm{mM}$ ) and control in frozen semen of bull (Uysal et al., 2007).

Uysal and Bucak, (2007) reported that many aspects of sperm protection like sperm motility, viability and membrane stabilization of sperm cells during relative cryopreservation are of prime importance. The antioxidants GSSG $(5 \mathrm{mM})$, BSA $(20 \mathrm{mg} / \mathrm{ml})$, Cysteine $(10 \mathrm{~mm})$ and Lycopene $(800 \mathrm{mcg} / \mathrm{ml})$ provide a near optimal concentration for improved bovine sperm survival during the freezing -thawing process. 
The sperm motility, viability and intact acrosome percent were significantly higher at all stages of refrigeration and preservation of triple cross breed bulls semen in the presence of EDTA $(0.1 \%)$ and Cysteine HCL $(0.1 \%)$ suggesting that any one of the two additives can be routinely added in the semen extenders with the beneficial results to sustain acceptable sperm quality for 3-4 days at $5^{\circ} \mathrm{C}$ temperature (Raval et al., 2007).

\section{Diluents with reduced Glutathione}

The excessive generation of reactive oxygen species (ROS) by abnormal spermatozoa and by contaminating leukocytes has been identified as one of the few defined etiologies for male infertility. Glutathione plays a significant role in the antioxidant defences of the spermatogenic epithelium, the epididymis, and perhaps in ejaculated spermatozoa. The use of antioxidants in-vitro appears to be of value in preserving fertilizing capacity. Glutathione administered in- vivo to patients who may have infertility, secondary to excessive oxidative stress, appears to act at the epididymis and during spermatogenesis; to improve the function of ejaculated spermatozoa (Irvine, 1996).

Levels of reduced glutathione were also significantly decreased in oligospermic and azoospermic group and the reduction in azoospermic group (76.73\%) was more pronounced than oligozoospermic group (62.07\%). The decrease in reduced glutathione, an endogenous antioxidant, in azoospermic and oligozoospermic conditions may cause disruption in the membrane integrity of spermatozoa as a consequence of increased oxidative stress (Bhardwaj et al., 2000).

The addition of glutathione (GSH, $1.5 \mathrm{mM}$ ) had an intermediate effect in preserving sperm motility at the end of the 30 -minute incubation $(53 \% \pm 3 \%)$. The decrease in sperm motility associated with ROS occurs in the absence of any detectable decrease in viability, acrosomal integrity and mitochondrial membrane potential or of any detectable increase in lipid peroxidation (Baumber et al., 2000).

Chatterjee et al. (2001) reported that Cryopreservation induces extensive biophysical and biochemical changes in the membrane of spermatozoa that ultimately decrease the fertility potential of the cells. Sulfhydryl groups of sperm proteins regulate a number of activities of the cells. Addition of glutathione reduced (GSH) or oxidized (GSSG) to the cryoprotectant partially prevented the effects of freezing/thawing, such as higher exposure of sulfhydryl groups, changes in the cellular distribution and enhanced rotational diffusion of sulfhydryl containing proteins of sperm membrane. Addition of GSSG to the cryoprotectant reduced by $35 \%$ the loss of motility of spermatozoa undergoing a freezing/thawing cycle. They concluded that cryopreservation perturbs 
sperm membrane sulfhydryl containing proteins and the modifications could partially be prevented by the addition of GSSG to the cryopreservation medium.

Bull sperm are exposed to aerobic conditions during processing before freezing and they have little endogenous antioxidant to protect them against reactive oxygen species that may be present. Antioxidants generally were not beneficial, except the percentage of motile sperm was improved by $6-11 \%$ units, when sperm were stored unfrozen or after freezing when $0.5 \mathrm{mM}$ of GSH with or without SOD was added. In field fertility trials, non-return rates were 71.9, 69.5 and 70.9\% with WM (whole milk) containing 0.0, 0.5 and 1.0mM of GSH, respectively, and 74.0 and $73.9 \%$ with WM and WM plus $0.5 \mathrm{mM}$ of GSH and $100 \mathrm{IU} / \mathrm{ml}$ of SOD (Foote et al., 2002).

Glutathione is a tripeptide and is widely spread among living organisms. It is present mainly in reduced form (GSH) and only a small amount is in oxidized form (CSSG). The role played by glutathione and its metabolism in bovine and bubaline semen is not yet well established. Glutathione (GSH) stimulates the aerobic glycolysis and respiration of epididymal sperm in semen. The metabolic regulation is due to $\mathrm{SH}$ groups of GSH. Addition of extraneous glutathione enhances the fertility rate of liquid and fresh semen (Jain and Arora, 2003).

In sperm pack, GSH concentration is higher in Red Dane bull as compared to indigenous and CB Bulls. Glutathione concentration in head and tail was comparatively higher in CB bull than other indigenous and exotic bulls. The GSH concentration was found to be highest in tail fraction following head and mid-piece in all the breeds. The comparative study between cow bull and buffalo bulls revealed that GSH values were higher in buffalo bulls as compared to cow bull sperm pack and various sperm fraction (Jain and Arora, 2003).

It has been reported that there was a loss in GSH content after cryopreservation of boar semen, addition of $5 \mathrm{mM}$ GSH to the freezing extender did not result in any improvement in either standard semen parameters or sperm fertilizing ability and addition of GSH to the thawing extender resulted in a significant increase in sperm fertilizing ability. GSH prevents damage of a sperm property that is critical in the fertilization process but that is not measured in the routine semen analysis (Gadea et al., 2004).

Glutathione is the most abundant antioxidant found in the body. It plays an important role in protecting lipids, proteins, and nucleic acids against oxidative damage. It combines with vitamin $\mathrm{E}$ and selenium to form glutathione peroxidase (Agarwal et al., 2005).

Chromatin condensation was equally affected by the addition of GSH. When GSH was added, a lower chromatin condensation was observed, as reflected by higher red fluorescence 
intensity and higher PI uptake (control: $78.11 \pm 2.32$ vs. $1 \mathrm{mM} \mathrm{GSH}: 89.24 \pm 2.28$ and $5 \mathrm{mM} \mathrm{GSH}$ : $92.85 \pm 3.42$ fluorescence units) The generation of ROS increased as a function of incubation time (30, 60, and 90 minutes), and ROS levels were significantly reduced following addition of GSH to the thawing media. Mean values of ROS generation for the GSH groups were close to $50 \%$ of the values of the control group (control: 140.45 vs. $1 \mathrm{mM} \mathrm{GSH:} 74.64$ and $5 \mathrm{mM}$ GSH: 66.32 fluorescence units). The addition of GSH to the thawing media reduced the percentage of capacitated viable sperm in a dose-dependent manner and reduced the percentage of viable spermatozoa with changes in the sulfhydryl groups in membrane proteins (Gadea et al., 2005b).

An important endogenous antioxidant in humans is the tripeptide glutathione (L-g-glutamylL-cysteinylglycine; GSH), which plays a central role in the defense against oxidative damage and toxins, where it serves as co-factor for glutathione peroxidase and glutathione S-transferases. Furthermore, GSH plays an important role in the protection against damage produced by oxidants, electrophiles and free radicals owing to its ability to react directly with hydrogen peroxide and superoxide anion, hydroxyl and alkoxyl radicals by its free sulphydryl groups (Eskiocak et al., 2005).

The main findings emerging from the addition of GSH (L-g-glutamyl-L-cysteinylglycine; GSH) to the thawing media were the lower number of capacitated viable spermatozoa, a decrease in the number of spermatozoa with changes in the sulfhydryl groups in membrane proteins, a reduction of the reactive oxygen species generation, a lower chromatin condensation, and a higher penetration ability of oocytes in vitro and a higher proportion of decondensated sperm heads. GSH appears to play an important role in sperm antioxidant defense strategy. Addition of GSH to the thawing extender could be of significant benefit in improving the function and fertilizing capacity of frozen boar spermatozoa (Gadea et al., 2005a).

Gadea et al. (2005a) reported that the effects of GSH supplementation of the freezing extender on sperm function to compensate for the reported decrease in GSH content during the sperm freezing. They have also reported that the addition of GSH to the freezing media resulted in an improvement in motility percent, motion parameters of thawed spermatozoa, higher number of total viable spermatozoa, higher number of non capacitated viable spermatozoa and the decrease in the number of spermatozoa with changes in the sulfhydryl groups in membrane proteins. This protective effect on sperm function was dose dependent, and it was more pronounced with $1 \mathrm{mM}$ of GSH than with $5 \mathrm{mM}$ of GSH. The addition of GSH to the media affects the percentage of sperm with low plasma membrane lipid packing disorder and the percentage of viable spermatozoa presenting a higher number of non capacitated sperm. 
The anti-oxidants treatments with GSH-10mM and Taurine $50 \mathrm{mM}$ provided a significant improvement in sperm motility, viability and membrane stabilization of the sperm cells during the 6 hours of liquid storage at $5^{\circ} \mathrm{C}$ (Bucak and Tekin,2006).

Cryopreservation reduces the spermatozoal GSH content. The level of total GSH of bovine spermatozoa detected in the raw semen ranged from 246.50 to 776.20 pmoles/mg of protein. The Bioxcell extender was superior in preserving GSH content $(647.20 \pm 300.1$ versus $223.60 \pm 154.0$ pmoles/mL; $\mathrm{P}<0.05)$, motility levels $(44.3 \pm 4.8$ versus $41.8 \pm 4.0 \% ; \mathrm{P}<0.05)$, non-capacitated state $(68.3 \pm 13.6$ versus $61.3 \pm 17 \% ; \mathrm{P}<0.05)$ and a lower proportion of acrosome reacted spermatozoa post thaw $(3.8 \pm 2.1$ versus $6.3 \pm 3.1 \% ; \mathrm{P}<0.001)$ compared to egg yolk tris-citrate extender. These findings could be partially ascribed to the high GSH level contained in the commercial extender which seems capable of alleviating oxidative damages to spermatozoa surviving freezing thawing procedures (Stradaioli et al., 2007).

Post thaw motility percent, total abnormality percent, acrosome damage percent, viability percent and HOST $(\%)$ values recorded are $60.6 \pm 1.63,10.9 \pm 2.45,3.0 \pm 0.73,78.6 \pm 4.48 \&$ $65.2 \pm 3.81(\mathrm{GSSG} 5 \mathrm{mM}), 45.00 \pm 3.42,19.20 \pm 4.76,11.70 \pm 3.17,70.50 \pm 5.29 \& 42.70 \pm 5.74$ (Cysteine $5 \mathrm{mM}$ )and:39.5 \pm 2.73, $30.10 \pm 5.30,13.40 \pm 2.32,50.70 \pm 6.05$ and $36.90 \pm 4.40($ Control) respectively in ram semen (Uysal and Bucak,2007).

The anti-oxidant treatments GSH@5-10 mM and taurine@50 mM provided a significant improvement in sperm survival, sperm motility, viability and membrane stabilization of the sperm cells during the 6 hours of liquid storage at $5{ }^{\circ} \mathrm{C}(\mathrm{P}<0.05)$ and they are the key factors determining the preservation of sperm function (Mustafa and Necmettin, 2007).

Uysal et al. (2007) reported that the antioxidants GSH $(5 \mathrm{mM})$ had a significant effect in maintaining sperm motility and membrane integrity, when compared to the control and other groups.

Bull semen diluents with Glutathione have been reported to improve post thaw motility and normal acrosome. It has also been reported to reduce enzyme leakage and consistently improved fertility. The mode of action as suggested might be to limit the production of lipid peroxides (Satish Kumar, 2007).

The mean progressive motility of spermatozoa in control and GSH-treated semen on day 2, $0.5 \mathrm{mM}$ GSH treated semen had significantly better sperm motility $(56.3 \%)$ than $0.0(55.4 \%)$ or 1.0 (52.9\%) mM GSH-treated samples. On Day 3 (54.0\%), 4 (46.4\%) and 5 (40.3\%), $0.5 \mathrm{mM} \mathrm{GSH}$ treatment showed significantly better sperm motility, but the concentrations of $0.0,2.0$ and $3.0 \mathrm{mM}$ 
GSH had 51.5\%, 49.6\% and 46.8\% sperm motility, respectively. Sperm motility in semen treated with $0.5,1.0,2.0$ and $3.0 \mathrm{mM} \mathrm{GSH}$, decreased by about $4 \%, 6 \%, 8 \%$ and $9 \%$, respectively, each day, but by about 6\% each day in the control group (Munsi et al., 2007).

It has been reported that the concentration of $5 \mathrm{mM} \mathrm{GSSG}$ was determined to exert the best protective effect on post-thaw sperm motility, sperm morphology, acrosome integrity, viability and membrane integrity in ram semen. Higher concentrations of GSSG (10 or $20 \mathrm{mM})$ may have exhibited negative or toxic effect on post-thawing spermatological indicators when compared to the $5 \mathrm{mM}$ concentration of GSSG (Uysal and Bucak (2007).

\section{Post Thaw Semen Evaluation}

Cryopreserved bovine spermatozoa, when used for artificial insemination, generally produced lower conception rates than fresh spermatozoa for which post thaw evaluation is one of the important factors in semen processing laboratories as motility, velocity, acrosome integrity and membrane stability all of which contribute to fertility.

Moustafa and Meszaros (1980) observed that semen collected from 69 AI bulls having good semen quality with good freezing capacity and good semen quality with poor freezing capacity had 50.0 and $21.5 \%$ post thaw motility respectively.

Wood et al. (1986) demonstrated through multiple regression analysis that a combination of sperm motility after dilution in saline, motility after thawing and the proportion of coiled tails and proximal protoplasmic droplets provide the best prediction of fertility with a significant correlation.

Belorkar et al. (1990) found that semen samples with 50\% post thaw motility or more were mostly from bulls with larger ejaculates, higher sperm concentration and lower percentage of abnormal and dead spermatozoa.

The acrosome integrity during semen storage have been studied by various workers and was found to vary from 74.05 to $83.70 \%$ prior to freezing to 69.40 to $76.10 \%$ after freezing (Nehring, 1988; Sharma et al., 2005).

Kjoestad et al. (1993) observed that the fertility of frozen bull semen to be more closely related to acrosome integrity than to motility with a low correlation between semen motility and acrosome integrity. The combined assessment of both these parameters had a significant effect on fertility, which would further emphasize the advantage of assessing both parameters in order to establish a reliable basis for post thaw estimation of semen quality. 
The ability to predict post thaw sperm quality and fertility from a routine sperm function assay would be beneficial, considering the extended period of progeny testing (Garner et al., 1994).

Nair, (1997) observed the mean post thaw motility and post thaw livability in half bred and three bred cross bulls to be $23.65 \pm 2.28,22.54 \pm 2.84,39.75 \pm 3.0$ and $40.42 \pm 3.38 \%$ respectively.

Brahmakshtri et al. (1999) established highly significant correlation $(\mathrm{P}<0.01)$ between post thaw motility and viability.

The reduced fertility of frozen thawed spermatozoa is thought to be not only due to a low sperm survival post thaw rate but also due to an impaired function of the surviving spermatozoa (Watson, 2000).

\section{Motility}

The mean post thaw motility was $47.14 \pm 0.92 \%, 28.33 \pm 5.26 \%, 44.16 \pm 2.99 \%, 42.65$ $\pm 1.54 \%, 23.64 \pm 5.325 \%$ and $28.33 \pm 2.35 \%$ for Pure JY, $75 \% \mathrm{JY}, 62.5 \% \mathrm{JY}, 50 \% \mathrm{JY}, 75 \% \mathrm{HF}$ and $62.5 \%$ HF bulls respectively (Sagdeo et al., 1980).

Mathew (1988) reported post thaw motility of 44.79\%. in 62.5\% Brown Swiss: and $44.79 \%$ in $62.5 \%$ JYCB. Similarly $50 \%$ JY CB bulls have recorded $43.33+1.86 \%$ post thaw motility (Tuli et al., 1988).

The pre-freeze and Post-thaw Motility (\%) in NLOP (Non-Luteal oviductal proteins, LOP (Luteal oviductal proteins) and control were 67.50 \pm 2.89 and 62.50 $\pm 2.89 ; 61.25 \pm 7.50$ and 52.50 $\pm 5.00 ; 52.50 \pm 2.89$ and $40.00 \pm 4.08$ respectively in the buffalo semen (Kumaresan et al., 2005).

The post thaw forward motility was significantly higher in diluents containing detergent mixture (4-6\%) as compared to caffeine puris control. This increased post thaw motility in frozen semen chelated in diluent containing detergent mixture could have happened because of the action of surfactants on the sperm cell membrane, making them more permeable and less sensitive to osmotic and glycerol shock during freezing and thawing. The over all post thaw motility and total abnormality in the thawed semen was $42.56 \pm 1.29$ and $14.26 \pm 0.04 \%$ respectively.

\section{Acrosomal Integrity (Giemsa Staining)}

Capacitated spermatozoa with 'intact' acrosomes may pass through the cumulus, but are unable to pass through the $\mathrm{ZP}$. The acrosome reaction, a modified form of exocytosis, is an absolute requirement for successful sperm passage through the ZP (Austin and Bishop, 1958). 
Tris dilutor readily penetrates the sperm cells and acts as an intracellular buffer, which reduces the damages caused to spermatozoa due to cold shock at ultra low temperature (Edwin et al., 1975).

The acrosome sustains damage during processing of semen and freezing or penetration of spermatozoa. The damage to the tune of 50\% may occur during dilution and cooling (Tasseron et al., 1977).

The mean value of (\%) intact acrosome 24 hours post thaw were higher in HF bulls $(86.00 \pm$ 1.13) than the CB bulls $(69.90 \pm 1.29)$. The extent of acrosomal damage was not significant between fresh and post thaw semen (Sharma et al., 1980).

There are a large number of plant lectins currently available that can be used to detect reacted acrosomes by attaching to exposed sugar moieties in the reacted acrosome compartment. A strong correlation between fertility and the percentage of bovine sperm exhibiting intact acrosomes has been previously reported (Berndtson et al., 1981).

The acrosomal content, containing mainly hydrolytic enzymes, starts to disperse and digest the ZP. The integrity of the acrosome can also be determined morphologically, usually at the light microscopy level, in unstained samples or with different empirical stains such as Giemsa. However, fluorescent probes are generally superior to colored dyes because, fluorescence provides greater intensity and greater contrast between acrosomal and non acrosomal material (Cross and Meizel, 1989).

The percentage of spermatozoa having an intact acrosome and being able to perform the acrosome reaction upon triggering is regarded as an important semen characteristic (De Leeuw et al. 1991).

Loss of acrosome structure was observed less in TEYFCAG (tris egg yolk fructose citrate glycerol) diluent and it was more pronounced in EYCFG (egg yolk fructose citrate glycerol). The intact acrosomes are 94.16 percent in fresh semen (pooled sample) which declined to 88.76 percent and 68.90 percent in pre- freeze semen dilutor in tris based diluents, where as it declined drastically to 85.98 percent and 56.70 percent in pre- freeze and post thaw semen respectively in citrate based dilutor (Singh and Purbey, 1996).

Fluorescent assessment of cellular integrity and mitochondrial function by flow cytometry can provide a rapid and precise means of determining the functional status of large numbers of 
spermatozoa. The rat sperm viability was assessed with SYBR-14 and PI and sperm mitochondria were differentially labeled with JC-1 (Curtis et al., 2000).

At 30 minutes time, there were no significant treatment effects on either viability or acrosomal integrity. The generation of ROS by the xanthine (X) - xanthine oxidase (XO) system had no effect on the percentage of live intact sperm $(90.2 \% \pm 1.7 \%)$ compared with that of control $(89.0 \% \pm 2.6 \%)$ (Baumber et al., 2000).

The acrosome reaction test is a valuable alternative method of determining the fertilizing ability of frozen thawed semen and in relation with motile and live spermatozoa (Pandey et al., 2004).

The over all mean \% of intact acrosome obtained in the semen of Gir and Jaffarabady bulls( $84.80 \pm 0.89$ and $83.50 \pm 1.24)$ did not vary significantly (Raina and Dhami, 2004).

\section{Acrosomal Integrity (FITC-PSA)}

The significant correlations between the percentage of spermatozoa with normal and intact acrosomes and the percentage of progressively motile spermatozoa were not unexpected since loss of the acrosomal contents under the incubation conditions used that would not likely be indicative of cell death (Garner et al., 1986).

It was found that FITC-RCA-Lectin binding can be an useful method for assessing the acrosomal status of fresh ram spermatozoa in suspension. This technique is the only described method by which the acrosomal status of unfixed ram spermatozoa can be assessed. Because it is simple and reliable, this acrosomal labeling technique should have broad applications in practical use such as the evaluation of sperm quality for cryopreservation or artificial insemination (Jose et al., 2000).

Specific labeling techniques have been used to enhance visualization of the acrosome with fluorescence microscopy. Lectins that bind to glycoconjugate of the acrosomal matrix or outer acrosomal membrane have been used to visualize the acrosome of human spermatozoa (Cross and Meizel, 1989) and that of bulls (Graham et al., 1990) and rams (Magargee et al., 1988).

Fluoresein-conjugated plant lectins such as fluorescein isothiocyanate-conjugated Pissum sativum agglutinin (FITC-PSA) have been used to selectively stain the acrosome of human spermatozoa (Tesarik et al., 1993) and that of stallions (Farlin et al., 1992), monkeys (Cross et al., 1988), bulls (Cross and Watson, 1994), and rams (Sukardi et al., 1997). 
Sperm plasma membrane is responsible for establishing a barrier between intracellular and extra cellular environments, which is important to maintain osmotic equilibrium and cellular homeostasis. Damages in this structure lead to cellular instability caused by homeostasis loss, resulting in cellular death. Therefore, plasma membrane integrity exerts a crucial role on sperm survival in the female reproductive tract and its fertilizing ability (Parks and Graham, 1992).

Acrosome integrity can be checked by different fluorescent techniques. Among them, the use of marked lectins is emphasized (Graham et al., 1990), which include Pisum sativum agglutinin (PSA), Ricinus Communis Agglutinin (RCA), Arachis hypogea agglutinin (PNA) (Cross and Meizel, 1989), and Concanavalia ensiformis (ConA), which are fluorescein isothiocyanateconjugates (FITC). PSA is an agglutinin from edible peas, and binds to the glycoconjugate of the acrosomal matrix (Cross and Meizel, 1989). It has affinity for terminal a-D-glucosyl and a-Dmannosyl residues of glycoproteins, and binds specifically to the sugar a-mannoside found in acrosomal contents (Sukardi et al., 1997).

This is an efficient and easy technique to simultaneously evaluate plasmatic, acrosomal, and mitochondrial membranes of fowl sperm. It is possible to suggest that the application of this methodology in large scale can be maximized by the use of flow cytometry systems, providing higher accuracy and swiftness, as it is to read approximately 10,000 cells in a few seconds (Celeghini et al., 2007).

The mean percentages of abnormal acrosome in treated and control groups of semen on $1 \mathrm{st}$ day, 2nd day, 3rd day, 4th day and 5th day were (@3.0 mM) 4.8 $\pm 0.3,8.3 \pm 0.6,11.7 \pm 0.7,15.4 \pm$ 0.9 and $20.5 \pm 1.0$ respectively. The proportion of acrosomal abnormality was significantly lower $(12 \%)$ in $0.5 \mathrm{mM} \mathrm{GSH}$ treated semen on Day 5 than in other groups (Munsi et al.,2007).

\section{Hypo Osmotic Swelling Test (HOST)}

Drevius and Eriksson (1966) demonstrated the swelling phenomena of sperm tail of bovine spermatozoa when exposed to a hypo osmotic solution $(0.024 \mathrm{M}$ to $0.043 \mathrm{M}$ sodium chloride solution).

Bredderman and Foote (1969) evaluated the proportion of swollen bovine sperm in hypotonic saline and found it to correlate almost significantly with 60 to 90 days non return rate.

In vitro fertilization assessment of hypo osmotic test using $150 \mathrm{~m} \mathrm{Osm} \mathrm{kg}^{-1}$ fructose / Trisodium citrate solution gave a very high correlation $(r=0.94)$ with the denuded hamster oocyte penetration test in human spermatozoa (Jeyendran et al., 1984). 
Hypo-osmotic swelling test was developed by Jayendran et al. (1984) to evaluate sperm membrane functional integrity and this test is based on the semi permeability of the intact cell membrane, which allows the sperm to swell under hypo-osmotic condition.

It was suggested that the ability of spermatozoa to swell in the presence of hypo osmotic medium reflects normal water transport across the sperm membrane, which is a sign of normal membrane integrity and functional activity (Jayendran et al., 1984).

Coetzee et al. (1989) observed that the correlation between hypo osmotic swelling and sperm viability was strong $(\mathrm{r}=0.76)$. A moderate correlation exists between hypo osmotic swelling and sperm morphology $(\mathrm{r}=0.50)$ and weaker correlation $(\mathrm{r}=0.42)$ with sperm penetration assay in human in vitro fertilization.

The functionally active spermatozoa exposed to a hypo osmotic stress swell due to influx of water and subsequently increase in volume to establish the equilibrium between the cytosol and the extra cellular milieu, spermatozoa with compromised or inactive membranes are unable to regulate water influx and remain not swollen. Thus, hypo osmotic swelling test may be useful in assessing changes in the sperm membrane functional integrity during freezing and thawing procedures (Revell and Mrode, 1994).

The spermatozoa, whose plasma membrane is intact, swell in response to hypo osmotic treatment $\left(150 \mathrm{~m} \mathrm{Osm} \mathrm{kg}^{-1}\right)$, a process seen as binding, coiling or shortening of the tail. Some of these swollen bovine spermatozoan cells continue to show activity. A good correlation existed between ability of spermatozoa to undergo capacitation, penetration of oocyte and hypo osmotic swollen positive spermatozoa percentage $(r=0.90, p<0.001)$. There exists a very little relationship between percentages of hypo osmotic swollen positive spermatozoa and percentage of normal sperm present in the ejaculates $(\mathrm{r}=0.61, \mathrm{P}<0.01)$. Relation between the percentage motile spermatozoa and swollen spermatozoa significantly correlated $(\mathrm{P}<0.01)$ with those that did not stain with Eosin - Y (Revell and Mrode, 1994).

Das (1994) observed that, total hypo osmotic swollen sperm in crossbred bulls varied from 49 to $72 \%$ with the average values being $62.13 \pm 2.76$ in a FBH, $64.75 \pm 0.99$ in FJH bulls. No significant difference was observed among the bulls and between the breeds by HOST.

The hypo-osmotic swelling test (HOST) was used as a complementary test to the viability assessment protocol to evaluate the functional integrity of the sperm plasma membrane. HOST relies on the resistance of the membrane to the loss of permeability barriers under stress conditions of stretching in a hypo-osmotic medium (Buckett et al., 1997). Sperm cells with resistant 
membranes exhibited a swelling around the tail, such that the flagella became curled and the membrane maintained a swollen 'bubble' around the curled flagellum.

Nair (1997) found the average post thaw hypo osmotic swollen positive sperm to be $23.54 \pm$ 1.41 and $23.83 \pm 1.2 \%$ in half bred and three bred cross bulls with a significant difference $(\mathrm{P}<0.05)$.

Post thaw motility $(37.4 \pm 4.32 \%)$ and HOST response $(50.69 \pm 2.07 \%)$ were significantly higher $(\mathrm{p}<0.01)$ for extender having 9\% glycerol compared to $6 \%$ glycerol. Longer equilibration period of 4 hours showed better post thaw motility $(32.4 \pm 4.42 \%)$ and HOST response $(48.67 \pm$ $2.07 \%$ ), when compared to 2 hours equilibration (Nair et al.,1999).

It is possible to evaluate using Eosin exclusion test, HOST, water test and fluorescent staining test with Propidium Iodide the structural and functional membrane integrity and viability correlated with the in-vitro fertilizing ability of sperms in frozen semen of boar and bull (Pintado et al., 2000).

The mean percentage of swollen buffalo spermatozoa following HOST post thaw were 45.0 $\pm 0.44,57.5 \pm 0.48$ and $45.5 \pm 0.41$ following conventional freezing, moderate rate and slow rate of programmable freezing respectively (Pant et al.,2002).

Pant et al., (2002) found that the lowest mean value for HOST positive spermatozoa also had the lowest fertility in buffaloes.

A negative correlation between HOS test results and ejaculate volume (bull: -0.278 , boar: 0.27 ) and a positive correlation between sperm cell concentration (bull: 0.216, boar: 0.43). This test is used to supplement commercial method of sperm evaluation (Madeja et al., 2003).

A significant positive correlation was observed between motility and swollen tail spermatozoa rates $(\mathrm{p}<0.01)$ and there was a correlation between tail defects and swollen tail spermatozoa rates $(\mathrm{p}<0.05)$ but acrosome, head, mid piece defects were not correlated with swollen tail spermatozoa $(\mathrm{p}<0.05)$ (Zekariya Nur et al., 2003).

A high significantly high and positive correlation was observed between sperm motility and sperm curling in fresh and frozen semen $(\mathrm{p}<0.01)$.the higher correlation between curling and motility than between curling and morphologic changes $(p<0.01)$ in frozen semen is not surprising. Since sperm motility depends partly on membrane transport (membrane integrity) and partially on other biochemical activities such as sperm metabolism (Uysal and Korkmaz, 2004). 
Hypo osmotic swelling - Eosin test is an easy assay practicable, useful and more accurate semen analysis method for predicting the fertilizing capacity of semen sample (Uysal and Korkmaz, 2004).

The HOST is not only an indicator of the chemical integrity of the plasma membrane, but also of its physical integrity (a broken membrane will not allow swelling to occur) therefore it is not necessary to perform the viability stain if the HOST is performed. This test is useful for detection of damage during cryopreservation, toxic effect of drugs, chemical and to assess quality of sperm (Sharma et al., 2005).

The mean HOST, water test, dead spermatozoa, motility \%, defective acrosome and total morphological defects were $53.7 \pm 11.3 \%, 49.8 \pm 9.7 \%, 12.7 \pm 5.7 \%, 62.5 \pm 8.65 \%, 7.2 \pm 2.9 \%$ and $9.7 \pm 3.4 \%$ respectively and mean concentration of sperm and $\mathrm{pH}$ were $2.2 \times 10^{9} / \mathrm{ml}$ and 6.8 respectively (Zekariya Nur et al.,2005).

There was a good correlation between motility and percentage of swollen tail spermatozoa obtained by both water and HOS tests, but no correlation was observed between swollen tail spermatozoa and dead sperms, acrosome defects, and total morphological defects (Zekariya Nur et al., 2005).

The overall response of spermatozoa in HOST solution was $33.67 \pm 1.25$ with a range of $30.50 \pm 1.00$ to $35.88 \pm 3.14 \%$. HOS positive sperm showed a positive correlation(r=0.98) with fertility rate and along with this test assessment of sperm morphological abnormalities could be useful in evaluating fertility of Frieswal bulls under field conditions (Mondal et al.,2007).

\section{Cervical Mucus Penetration Test (CMPT)}

Cervical mucus plays an important role in selecting motile, mostly morphologically normal sperm for fertilization, so that CMPT has always held as an important in-vitro sperm functional test. Bovine cervical mucus is particularly common because it is easier to obtain in large quantities and has similar rheological and biochemical properties to human cervical mucus and can be stored in the frozen storage with only minimal changes in rheological properties (Lee et al., 1981).

Amit et al. (1982) used average swim up sperm velocity, which is directly related to sperm count per high power field at 10,20,30 and $40 \mathrm{~mm}$ after 60 minutes. Cervical mucus is a physiological barrier controlling sperm access to the upper female reproductive tract. Spermatozoa with anomalies of the flagellum and particularly of the middle piece are not able to migrate through the cervical mucus (Jeulin et al., 1985). Furthermore, the pattern of sperm movement and amplitude 
of the lateral head displacement are also very important for cervical mucus penetration (Aitken et al., 1985).

Spermatozoa are selected both quantitatively and qualitatively during their migration through the cervical mucus and during their transit in the female genital tract (Jouannet and Feneux, 1987).

During natural mating, spermatozoa must migrate through the cervical mucus to enter the uterus. Subsequently, spermatozoa are transported mainly by muscular movements of the uterus to the functional sperm reservoir in the oviduct (Suarez et al., 1990).

The ideal male fertility should provide some assessment of sperm function as well as measurement of concentration and morphology. The in-vitro sperm mucus penetration test is a sperm function test which measures the ability of sperm in the semen to swim up into a column of cervical mucus or its substitute. If it can be proven to be as good as semen analysis in assisting progressive sperm motility, then arguably, its additional benefit as a test of functional competence may make it a suitable and cheaper alternative to the present combination of semen analysis and sperm separation procedure. This attraction has led to the re emergence of research into CMPT for the assessment of semen. Sperm migration into cervical mucus is based on the same principle as the Kremer test (Kremer et al., 1995).

The sperm penetration speed (SPS) for the different bulls varied between $62.64 \pm 2.65 \mu / \mathrm{Sec}$ and $48.61 \pm 1.97 \mu /$ Sec. The SPS for different cows varied between $69.72 \pm 1.84 \mu / \operatorname{Sec}$ and 43.75 $\pm 0.79 \mu / \mathrm{Sec}$. The sperm penetration speed was significantly different between the bulls $(\mathrm{p}<0.01)$ and between the different mucus samples $(\mathrm{p}<0.01)$ as reported by Anil Kumar and Devanathan (1995).

Some of the parameters used to reflect the degree of penetration include, the migration or vanguard distance (distance between the foremost sperm in the capillary tube to the end of the tube in the semen reservoir), swim up sperm count/sperm velocity at 10, 20, 30 or $40 \mathrm{~mm}$ at high power field and migration reduction. Of these, most commonly used parameter is the vanguard distance of $30 \mathrm{~mm}$, measured after incubation at $37^{\circ} \mathrm{C}$ for 90 minutes. In addition, there might be technical difficulties with filling capillary tubes with cervical mucus (Tang et al., 1999). Although sperm migration has been extensively refined and validated in human infertility (Clarke et al., 1998). Studies on its application to predict bovine fertility has been very limited and a clear relation between the sperm migration distance and in vivo bull fertility has never been demonstrated. 
It has been found that $\mathrm{SMPT}_{\mathrm{vd}}$ has a low accuracy in the evaluation of semen, but $\mathrm{SMPT}_{\mathrm{sc}}$ was found to be moderately accurate in assessing sperm motility in semen. This method of using sperm concentration, instead of vanguard distance, as diagnostic criteria of in-vitro SMPT has got potentiality as an useful laboratory based sperm function test (Bolarndeola et al., 2003).

The highest repeatability of the sperm migration test $(\mathrm{CV}=10.7 \%)$ was obtained when the sperm migration distance of the five vanguard motile spermatozoa was determined at $30 \mathrm{~min}$ incubation at $37^{\circ} \mathrm{C}$ in a migration medium with $1.35 \% \mathrm{MC}$. No significant difference in migration distance was demonstrated when sperm concentrations of $100 \times 10^{6}$ and $150 \times 10^{6}$ spermatozoa $/ \mathrm{ml}$ were used. Despite the relatively high repeatability of the migration test, no relation was found between the sperm migration distance and the 56day-NRRs of five sire bulls. Therefore, the sperm migration test in $1.35 \%$ MC cannot be used to predict in vivo bull fertility accurately (Steven, 2004).

The pregnancy outcome from the bulls having maximum SPD(sperm penetration distance) in bovine cervical mucus (fresh semen $-38.63 \pm 0.26$, extended semen-37.39 \pm 0.19 , frozen semen$36.85 \pm 0.21$ ), $2 \%$ PAG fresh-37.44 \pm 0.54 , extended-36.60 \pm 0.34 , frozen-35.89 \pm 0.44 were found $58.83 \%$ where as bulls showing lower values of SPD in BCM and 2\% PAG had lower pregnancy outcomes (Bhatia et al.,2007).

The mean distance traveled by fresh and frozen thawed spermatozoa in bovine cervical mucus was $26.80 \pm 0.65$ and $29.40 \pm 1.00 \mathrm{~mm} / 60 \mathrm{~min}$ respectively, with an increase in the mean value by $16.8 \%$ from fresh to frozen thawed stage. The frozen thawed spermatozoa covered significantly high distance in the cervical mucus than that of fresh spermatozoa. This indicates that the cervical mucus penetration test is a good indicator to predict the functional status of frozen thawed spermatozoa (Satish Kumar and Kadirvel, 2007).

\section{Mitochondrial Membrane Potential (MMP)}

A combination of 6-carboxyfluorescein diacetate (CFDA) and Propidium iodide (P1) could provide quantifiable information on functional aspects of spermatozoa due to their molecular characteristics. The CFDA fluorophore, which readily permeates membranes, is non-fluorescent. But, hydrolysis of the ester bonds results in formation of a highly fluorescent, membraneimpermeant green fluorophore. Intracellular esterases readily hydrolyze CFDA and the resultant product, 6-carboxyfluorescein, is trapped within cellular structures possessing intact membranes. The counter stain, P1, is a bright red, nucleic acid-specific fluorophore. This fluorophore is relatively impermeable to membranes and thus does not readily stain nuclei of viable spermatozoa. 
Thus, a combination of dual fluorescent staining and flow cytometric analysis may make it possible to quantitatively assess spermatozoan viability and potential fertility (Garner et al., 1986).

The greater variability seen in the evaluations of motility are reflective of the limitations inherent in this visual method of quantifying the percentage of motile spermatozoa as used routinely by most semen-processing organizations. Mitochondrial membrane potential is widely used for characterization of cellular metabolism, viability and apoptosis in various cellular models. In human, a correlation exists among poor sperm mitochondrial function, diminished motility and reduced fertility (Marchetti et al., 2002 and Wang et al., 2003).

For determination of mitochondrial transmembrane potential, the dye -5, 5', 6, 6'-tetrachloro1, 1', 3, 3'-tetraethylbenzimidazolyl carbocyanine iodide, JC-1 can be used. JC-1 is a dye, which fluoresces green in monomeric form upon initial entry into the cytoplasm. Oxidation within the mitochondria causes the formation of J-aggregates; these fluoresce red-orange and are concentrated within the mitochondria. The most widely used mitochondrial-specific probe (JC-1), is capable of entering selectively into mitochondria, since it changes reversibly its color from green to orange as membrane potentials increase (over values of about $80-100 \mathrm{mV}$ ). This property is due to the reversible formation of JC-1 aggregates upon membrane polarization that causes shifts in emitted light from $530 \mathrm{~nm}$ (i.e. emission of JC-1 monomeric form) to $590 \mathrm{~nm}$ (i.e. emission of J-aggregate). When excited at $490 \mathrm{~nm}$; the color of the dye changes reversibly from green to greenish orange as the mitochondrial membrane becomes more polarized. The phenomenon producing the red-orange fluorescence has been referred to as J-aggregate formation. The proportion of red-orange and green fluorescence (aggregates: monomers) depends on the mitochondrial membrane potential. Mitochondria with high membrane potential fluoresce red-orange, while those with low to medium membrane potential fluoresce green. While JC-1 indicates the presence of membrane potentials, JC1 has the unique property of further differentiating between high and low membrane potentials.

The formation of aggregates depends on the chemical environment of dye concentration, $\mathrm{pH}$, ionic strength, and temperature. Fluorescent mitochondria may not have been the sole indicator of sperm motility. It is possible that mitochondrial fluorescence quenched or failed to fluoresce at very low levels while the spermatozoa continued to exhibit relatively slower progressive motility. The biological significance of the proportions of aggregates and monomers in spermatozoa remains to be resolved.

In human, a correlation exists between poor sperm mitochondrial function detected by reduction of $\Delta \Psi_{\mathrm{m}}$ and diminished motility and reduced fertility (Marchetti et al., 2002 and Wang et 
al., 2003). Therefore a greater understanding of the molecular basis of male fertility is essential for sperm egg interaction and embryo development.

Mitochondria are the major organelles for the production of adenosine triphosphate (ATP) required for sperm motility and ATP production is very important for maintaining flagellar movement for a long period of time. This is required for sperm to reach the site of fertilization (Bartoov et al., 1980).

The functional status of sperm organelles or compartments can be assessed by organelle specific staining. The mitochondrial function of spermatozoa can be assessed using JC-1, a dye that accumulates in mitochondria as J-aggregates| and fluoresces red-orange when the membrane potential $(\Delta \psi)$ is relatively high, or as the monomeric form that fluoresces green when the $(\Delta \psi)$ is relatively low (Reers et al., 1991).

The mitochondrial probe 5,5',6,6'-tetrachloro-1,1',3,3'-tetraethylbenzimidazolyl-carbocyanine iodide (JC-1) not only identifies mitochondria exhibiting low membrane potentials by the emission of green fluorescence (range, 510-520 nm) but also differentiates these from mitochondria exhibiting relatively high membrane potentials. This discrimination occurs because JC-1 forms aggregates at high membrane potentials. These J-aggregates emit a bright red-orange fluorescence at $590 \mathrm{~nm}$. Thus, JC-1 was combined with the classical dead cell stain, Propidium iodide (PI), to identify a spectrum of functional sperm along with degenerate sperm (Garner et al., 1999).

The effects of stain equilibration time, sperm concentration, and live and dead ratio were examined. The addition of SYBR-14 to the JC-1 and PI combination enhanced the distinction between the red PI-stained and red-orange JC-1-stained populations. This discrimination between Jaggregates and the PI-stained sperm was affected by sperm concentration. These indicate that JC-1 can be useful in monitoring mitochondrial function in bovine sperm (Garner et al., 1999).

Motile sperm stained with JC-1 appeared orange in the mid-piece indicating a high mitochondrial membrane potential, whereas immotile sperm with a low membrane potential stained green. The percentage of spermatozoa staining orange was highly correlated with expected sperm viability (Curtis et al., 2000).

There were no significant treatment effects on mitochondrial membrane potential after incubation with the xanthine $(\mathrm{X})$ - xanthine oxidase $(\mathrm{XO})$ system. The generation of ROS by 0.6 $\mathrm{mM} X-0.05 \mathrm{U} / \mathrm{mL} \mathrm{XO}$ had no effect on orange (9133 relative fluorescence units RFU) or green (15 798 RFU) fluorescence measured on the multi well plate reader compared with the case of the control (8631 and $14222 \mathrm{RFU}$, respectively). The generation of ROS by $0.6 \mathrm{mM} \mathrm{X}-0.05 \mathrm{U} / \mathrm{mL}$ 
$\mathrm{XO}$ had no effect on the percentages orange $(88 \%)$ or green $(11 \%)$ populations determined by flow cytometry, compared with the case of control ( $88 \%$ and $11 \%$, respectively) (Baumber et al., 2000).

The high mitochondrial membrane potential was correlated with fertility as compared to reactive oxygen species production, DNA fragmentation and viability (Marchetti et al., 2002).

The sperm mitochondrial membrane potential increases leads to sperm motility parameters and fertility potential will also increase. The JC-1 dye method is useful to predict sperm fertility potential (Kasai et al., 2002). Changes in the mitochondrial membrane potential have been proposed to be a good indicator of sperm functional impairment (Pena et al., 2003).

Guillaume et al. (2004) has reported that cryopreservation had a dramatic effect on spermatozoon $\Delta \psi \mathrm{m}$. The synthesis of ATP is under mitochondrial control and dependent on a high $\Delta \psi \mathrm{m}$. A $\Delta \psi \mathrm{m}$ decrease can result in mitochondrial dysfunction, leading to non-renewal of ATP. Then a lack of energy and depletion in ATP can be responsible for the decreased spermatozoon cell motility, usually observed after cryopreservation and thawing.

Motility study is currently the most commonly used tool to evaluate sperm quality in commercial bull stations. At best it is an indicator or an indirect measure of metabolic activity and cell viability (Hallap et al., 2005).

The mean \pm SE and range of Progressive forward motility (\%), Acrosomal integrity (\%), Sperm nuclear morphology (\%) and Mitochondrial membrane potential (\%) were $51.67 \pm 2.69 \&$ $41.67-60.00,77.51 \pm 7.50 \& 40.50-90.74,84.64 \pm 0.86 \& 83.22-88.56$ and $19.65 \pm 3.18 \& 5.90$ 27.81 respectively in buffaloes (Selvaraju et al.,2007).

Functional membrane integrity, subpopulation of spermatozoa positive for functional membrane integrity, acrosomal integrity and plasmalemma integrity measured by fluorogenic staining and mitochondrial membrane potential had significant contribution to the sperm zona binding and penetration (Selvaraju et al., 2007).

\section{Lipid Peroxidation Assay (LPO)}

The mechanism by which lipid peroxidation produces inert spermatozoa to cause damage to the sperm membrane, which results in leakage in intracellular constituents, including adenine and pyridine nucleotides and enzymes. Without intracellular ATP, there can be no motility. It would therefore be expected that the percent inert sperm produced by this mechanism would correlate with the extent of lipid peroxidation and not with the time of exposure to aerobic incubation. It is important to note the distinction between the effect of the ionic composition of the medium on lipid 
peroxidation, which irreversibly converts motile sperm to inert sperm, and on patterns of motility, including reversible loss of motility (Alvarez and Storey, 1982).

Spermatozoa from several mammalian species, including mice and humans, are highly susceptible to oxygen induced damage mediated by lipid peroxidation because of their high content in polyunsaturated fatty acid and relatively low levels of antioxidants (Aitken et al., 1989). Exposures to fatty acid peroxides or to high concentrations of the combination of ferrous iron and ascorbic acid (Fe2+/Asc) to induce excessive lipid peroxidation in sperm membranes result in a rapid loss of motility and viability (Jones et al., 1979). Similarly, incubations under conditions that favor oxygen contact with spermatozoa result in high levels of lipid peroxidation and cell death (Alvarez et al., 1987).

Sperm cells have a high content of unsaturated fatty acids in their membranes but lack in significant cytoplasmic component containing antioxidants. Therefore, sperm cells are highly susceptible to lipid peroxidation (LPO) by free radicals such as hydrogen peroxide, superoxide anion, and hydroxyl radical, which lead to the structural damage of sperm membranes during the freezing-thawing process (Sinha et al., 1996).

The thiobarbituric acid (TBA) assay has been widely used to provide a convenient index of lipid peroxidation, the outcome of which correlates well with alternative techniques for assessing peroxidation, including chemiluminescence, pentane or ethane formation and colorimetric reactions based on the reduction of phosphoilpid hydroperoxides with potassium iodide (Jones et al., 1979).

Despite a certain lack of selectivity (Bird and Draper, 1984) have arguments in favor of using the TBA-assay to give a general indication of the extent of lipid peroxidation in mammalian spermatozoa (Alvarez and Storey, 1982 and Alvarez et al., 1987).

As a consequence, spontaneous Malondialdehyde production by human spermatozoa is slow and long incubation periods, have to be used to monitor the peroxidation process (Alvarez et al., 1987).

The hydrogen peroxide might be more likely to affect intracellular enzyme systems because of its higher membrane permeability and alternatively, enzyme inhibition could be induced indirectly by products of lipid peroxidation, especially Malondialdehyde and 4-hydroxynonenol. Low concentrations of these substances like Malondialdehyde and 4-hydroxynonenol have been shown to inhibit a large number of cellular enzymes and functions like anaerobic glycolysis, DNA, RNA, and protein synthesis (Comporti, 1989). 
Lipid peroxidation occurring in both the seminal plasma and spermatozoa was estimated by Malondialdehyde (MDA) concentration. Spermatozoal MDA concentration was higher in men with decreased sperm motility. The MDA concentration in the seminal plasma exhibited no relationship with sperm concentration, sperm motility, the number of immotile spermatozoa, or even the absence of spermatozoa. The MDA concentration in sperm pellet suspensions of asthenospermic and oligoasthenospermic patients was almost twice that of the normospermic males. The MDA concentration in the sperm pellet suspension from normospermic or oligospermic patients was about $10 \%$ of the seminal plasma content. However, the MDA concentration in the sperm pellet suspension of asthenospermic or oligoasthenospermic patients was about $15 \%$ that in the seminal plasma. Treatment of asthenospermic patients with oral Vitamin E significantly decreased the MDA concentration in spermatozoa and improved sperm motility (Suleiman et al., 1996).

Malondialdehyde was increased $(0.82 \pm 0.51 \mu \mathrm{M})$ significantly above the control $(0.43 \pm$ $0.19 \mu \mathrm{M})$ with the addition of xanthine $(\mathrm{X})$ - xanthine oxidase (XO) system at the highest concentration (Baumber et al., 2000).

Hydrogen peroxide has a primary role in mediating equine spermatozoa damage due to ROS generation by the xanthine $(\mathrm{X})$ - xanthine oxidase (XO) system. Equine sperm motility is a more sensitive indicator of oxidative stress than are viability, acrosome integrity, lipid peroxidation, and mitochondrial membrane potential. It has also been suggested that equine sperm motility may be affected by a pathway of ROS action independent of lipid peroxidation and mitochondrial membrane dependent functions, both previously reported to be responsible for ROS damage to motility in human spermatozoa (Baumber et al., 2000).

It is also possible that the assay for lipid peroxidation was not sensitive enough to detect small changes in lipid peroxidation that may have affected sperm cell function, such as in the mitochondrial membrane or mitochondrial enzymes (Baumber et al., 2000).

The MDA production in one group increased from $1.17 \pm 0.29$ at $0 \mathrm{~h}$ to $7.50 \pm 1350.52$ $\mathrm{nmol} / 10^{8}$ spermatozoa after $72 \mathrm{~h}$ while in other group it increased from $1.99 \pm 0.26$ to $8.70 \pm 0.10$ $\mathrm{nmol} / 10^{8}$ spermatozoa during the same period. Spermatozoa of buffalo bull showed a significant higher $(\mathrm{p}<0.05)$ LPO at $0 \mathrm{~h}$ as well as at 12,24 and $48 \mathrm{~h}(\mathrm{p}<0.01)$ of storage at refrigeration temperatures than spermatozoa of cattle bull (Sreejith et al., 2005).

\section{DNA Fragmentation}

Sperm with non-compensable defects such as abnormal DNA distribution has been associated with improper zygotic, embryonic and or fetal development (Saacke, 1998). Since sperm 
DNA is uniquely condensed and organized, any abnormality associated with DNA, chromatin packing, or the sperm nuclear matrix should be reflected by a change in sperm nuclear shape. The evaluation of sperm DNA distribution should be useful for assessing male fertility.

The DNA-damaged sperm (regardless of degree of damage) have the ability to fertilize the oocyte, but that embryonic development is very much related to the degree of DNA damage. However, the oocyte has the capacity to repair DNA damage of sperm when it is damaged by less than $8 \%$. Damage beyond this level will result in low rate of embryonic development and high early pregnancy loss (Ahmadi, 1999).

Many of the reproductive toxic agents have been studied to exhibit cell-type specificity resulting in increased DNA fragmentation in epididymal or ejaculated sperm. Thus, it appears that there are various mechanisms that result in DNA strand breaking in mature sperm. One of the primary DNA damaging agents receiving a lot of research attention is reactive oxygen species (ROS) (Henkel et al., 2003).

Henkel et al. (2004) showed no correlation with fertilization rates, pregnancy, DNA integrity or DNA fragmentation using early markers of apoptosis (Annexin V binding and Fas expression). As there is clear relationship between high DNA fragmentation and lower pregnancy rates, it seems possible that DNA damage may be due to ROS.

Harmful metabolites from polycyclic aromatic hydrocarbons in polluted air are detoxified by glutathione s-transferase and the men who lacked the gene for the enzyme glutathione stransferase would be more susceptible to the effects of air pollution resulting in sperm DNA damage (Evenson and Wixon, 2006).

The lower DNA damage observed following supplementation of the thawing medium with GSH, suggests that ROS generation and redox balance are the most important factors responsible for the disruption of the condensation and stability of sperm chromatin after cryopreservation (Joaqui et al., 2007). DNA fragmentation index in Control , $1 \mathrm{mM} \mathrm{GSH}$ and $5 \mathrm{mM}$ GSH were $1.2 \pm$ $0.1,0.8 \pm 0.1$ and $0.8 \pm 0.2$ respectively (Joaqu et al., 2007)

Bollwein et al. (2008) analyzed DNA fragmentation by the standard deviation of the DNA fragmentation index (SD-DFI) and the percentage of sperm with a high degree of DNA fragmentation $(\% \mathrm{DFI})$ by using $\mathrm{SCSA}^{(\mathrm{TM})}$.

The mean non-return rate on day 56 (NRR 56) ranged from $63.7 \%$ to $78.0 \%$ (mean +/- SD: $71.8 \%+/-3.7 \%$ ). Mean values for PMI (plasma membrane intact sperm) and HMMP (high 
mitochondrial membrane potential) decreased from $37.4 \%+/-6.8 \%$ to $31.2 \%+/-6.1 \%$ and from $38.8 \%+/-7.1 \%$ to $23.8 \%+/-7.7 \%$ respectively. SD-DFI (standard deviation of the DNA fragmentation index) increased from $56.9 \%+/-8.0 \%$ to $69.0 \%+/-12.9 \%$ and $\%$ DFI from $6.4 \%+/$ $2.5 \%$ to $12.4 \%+/-5.8 \%$ (Bollwein et al., 2008).

The lower DNA damage observed following supplementation of the thawing medium with GSH suggests that ROS generation and redox balance are the most important factors responsible for the disruption of the condensation and stability of sperm chromatin after cryopreservation. The DNA damage induced by ROS generation could negatively affect the fertilization rate and early embryonic development. The DNA damage induced by ROS generation could negatively affect the fertilization rate and early embryonic development (Joaqu et al., 2007).

Free radicals are known to be involved in lipid peroxidation as well as DNA and sperm membrane damages that may lead to decreased sperm motility or cell death (Uysal et al., 2007).

Cryopreservation caused a significant but low (3.8\%) decrease in the percentage of DNA in the comet head and an increase (5.3\%) in the tail length. This indicates that in addition to motility and viability, low levels of DNA fragmentation after cryopreservation is a characteristic of bull spermatozoa and can be a part of remarkable cryoresistance of bull spermatozoa (Słowińska et al., 2008).

\section{Computer Assisted Sperm Analysis (CASA)}

Attempts have been made to develop other techniques to measure the motility of spermatozoa to avoid inevitable human bias. The methods used to estimate sperm velocities have ranged from very simple techniques, such as time-exposure or multiple exposure photomicrography, to the very sophisticated computer-assisted sperm analysis (CASA) techniques (Mortimer, 1997). Recent findings suggest that it is not just the proportion of motile spermatozoa that is of great importance. The objective and quantitative measurement of other sperm motion characteristics derived from observations of individual cells, assessed by CASA, have been found to be more predictive of a sample's potential fertility (Mortimer, 1994). In the case of the bovine, specific motion parameters have been reported to be related to fertility (Budworth et al., 1988; Farrell et al., 1998). In addition to the use of computerized techniques to predict semen fertility, CASA provides an useful tool to study the effects of various in vitro procedures on sperm motility, as well as the means to study the phenomenon of sperm hyper-activation.

Computer assisted semen analysis (CASA), a tool used for providing quantitative data on sperm motility, gives more objective and detailed spermatozoa kinematics information than 
traditional methods do (ESHRE Andrology Special Interest Group, 1998). There is ample evidence to indicate that CASA parameters can be used to predict fertility (Macleod and Irvine, 1995). Sperm velocity and motility are among the most important parameters of semen quality because they are correlated with fertility (Aitkin, 1990).

CASA can be used as a predictive tool for fertility in human post thaw spermatozoa. The main variables included for semen fertility in CASA parameters are ALH and VAP (Macleod and Irvin 1995). ALH remains a very useful parameter because it reflects the amplitude of the proximal flagellar wave (ESHRE Andrology Special Interest Group, 1996).

Fertilization rates are related to ALH, VCL, VSL, and rapid sperm movement, with motility (rapid) and VCL being the 2 most important parameters (Hirano et al, 2001). Motility, LIN, curvilinear velocity, and average path velocity — but not VSL-may serve as prognostic indicators for the fertilization potential of sperm (Joshi et al, 1996). A strong correlation exists between morphology, VCL, VSL, ALH, and motility but not LIN (Stachecki et al., 1993). In human, a VCL greater than $25 \mathrm{~mm} / \mathrm{s}$ was reported to be the most significant and independent CASA parameter for the prediction of male fertility potential (Larsen et al., 2000).

Various kinds of motion characteristics have been reported in many species including bovine (Anzar et al., 1991; Budworth et al., 1987), equine (Jasco et al., 1988) and canine (Ellington et al., 1993) using the CASA system. A high correlation coefficient between motility or velocity of bull spermatozoa and competitive fertility index was reported by Budworth et al. (1987). Significant positive correlations among HOST, MOT, VSL, VCL, ALH and VAP indicated the dependency of sperm kinematics on plasma membrane integrity (Mandal et al., 2003). The VSL of spermatozoa has been correlated with fertilization rates, and differences between the VSL of sperm from fertile and sub fertile males has been reported (Leidel et al., 1993; Liu et al., 1991; Wainer et al., 1996). The VSL of sperm is an indication of spermatozoa flagellar motion and thrust. These sperm-motion characteristics have been shown to be important in penetration of the zona pellucida (Katz et al., 1989) and in transport through oviductal mucus (Suarez et al., 1992).

Hyperactive motility of mammalian spermatozoa is regarded as important for penetrating the zona pellucida of oocytes (Yanagimachi, 1994) and has therefore been proposed for assessment of semen quality, particularly in men (Burkman, 1990; Mortimer, 1997 and Kay and Robertson, 1998). CASA requires threshold values to differentiate between non-hyperactive and hyperactive spermatozoa. The values reported for human (Mortimer and Swan, 1995 and Mortimer, 1997), ram (Mortimer and Maxwell, 1999) and rat (Cancel et al., 2000) spermatozoa are different and reflect the species-specific patterns of sperm movements. Nevertheless, the commonly used parameters to 
identify hyperactive spermatozoa are a decrease in curvilinear velocity (VCL) and linearity (LIN) as well as an increase in lateral head displacement (LHD) (Mortimer 1997). For boar spermatozoa a decrease in VSL and an increase in VCL, resulting in a reduced linearity, were reported upon hyperactivation (Suarez et al., 1992). Although there are some differences in absolute values, especially in VCL after hyperactivation, the tendency of the changes upon hyperactivation has been confirmed.

When measuring sperm velocity, CASA evaluates 3 motility characteristics; velocity average path (VAP), velocity straight line (VSL) and curvilinear velocity (VCL). Curvilinear velocity is the total distance in an observation period that the sperm head can traverse, and it has the largest numerical value of the three velocities. From one point to another, VSL measures the straight-line distance in an observation period and has the smallest numerical value. Average path velocity measures the distance the spermatozoa has traveled in a general direction in a given observation period. Another useful measurement is the beat cross frequency (BCF), which counts the number of times that the sperm head crosses the direction of movement. Beat cross frequency is a valuable measurement because it assesses the number of times the flagellar beat changes its pattern. The average amplitude of the lateral head displacement (ALH) measures the width of movement made by the lateral head of the spermatozoa. Linearity (LIN) is evaluated by looking at both VSL and VCL and then dividing VSL by VCL. The technology of CASA has been used in studies exploring sperm motility measurements of many species such as rats (Moore and Akhondi, 1996), bulls (O’Conner et al., 1981), boars (Holt et al., 1997), rabbits (Farrell et al., 1993), turkeys (Bakst and Cecil, 1992), and humans (MacLeod and Irvine, 1995).

Sperm motility in toms measured by CASA showed a higher VSL and VAP in high-mobility spermatozoa than in average- or low-mobility spermatozoa (Donoghue et al., 1998).

The fresh semen of boars (Vizcarra and Ford, 2006) and turkeys (King et. al., 2000) showed a significant correlation between sperm mobility and CASA measurements (PGM, BCF, VAP, VCL, and VSL), where as with stored semen, there was no correlation. This finding indicates that higher-mobility sperm swam faster than the low-mobility sperm as indicated by BCF, VSL and LIN being velocity measurements (King et al., 2000).

Significant differences were found between the high and the low groups in \%MOT (sperm motility) $(91.1 \pm 8.5$ vs $63.0 \pm 32.7$, mean \pm SD), VAP $(73.0 \pm 14.2$ vs $52.1 \pm 12.5)$, VCL $(127.0 \pm$ 28.1 vs $87.0 \pm 22.6), \%$ HA (hyper activated sperm) $(27.3 \pm 23.6$ vs $7.2 \pm 9.0)$ and \%FR [73.2 $(48 / 56)$ vs $59.0(69 / 117)]$. No significant differences were found in other CASA parameters (Kasai et al., 2002). 
Progressive motility (\%), Total motility (\%), VCL, VSL, VAP, LIN, STR, WOB, ALH and $\mathrm{BCF}$ at Control 40.2 $\pm 1.2,59.0 \pm 1.4,115.8 \pm 2.0,87.1 \pm 2.2,98.3 \pm 2.1,74.0 \pm 0.787 .1 \pm 0.4$, $83.9 \pm 0.5,2.7 \pm 0.16 \& 6.1 \pm 0.1$ and at $1 \mathrm{mM} \mathrm{GSH}$ were $41.00 \pm 1.4,61.8 \pm 1.6,114.0 \pm 2.1,84.2$ $\pm 2.0,96.9 \pm 2.1,72.3 \pm 0.7,85.4 \pm 0.5,83.7 \pm 0.4,2.87 \pm 0.0 \& 5.8 \pm 0.1$ and at $5 \mathrm{mM} \mathrm{GSH} 43.6$ $\pm 2.0,59.3 \pm 1.3,118.5 \pm 2.00,88.7 \pm 2.1, \quad 100.3 \pm 2.173 .6 \pm 0.7,87.1 \pm 0.4,83.5 \pm 0.5,2.8 \pm$ 0.0 and $6.1 \pm 0.1$ respectively (Joaqui et al.,2007).

Before swim-up, there were significant correlations between fertilization rates and CASA estimates, including amplitude of lateral head displacement (ALH), curvilinear velocity (VCL), straight line velocity (VSL) and rapid sperm movement (Rapid). There was also a significant correlation between the fertilization rates and straightness (STR) after swim-up. As for sperm movement characteristics, there were significant differences of ALH $(p<0.005)$, VCL $(p<0.001)$, VSL $(p<0.005)$ and Rapid $(p<0.01)$ between "good" and "poor" groups before swim-up. After swim-up, there were significant differences of VCL $(p<.0005)$, average path velocity (VAP) (p $<0.005$ ), and Rapid $(\mathrm{p}<0.05)$ between the two groups (Hirano et al., 2001).

Percentage motility (by CASA) ranged from 33.08 in the control group to 37.98 in the $5 \mathrm{mM}$ GSH group. Most of the motion parameters were affected by the freezing batch, but they presented a similar pattern under the same treatments. Although no significant differences were found for percentage of motility (both CASA and microscopic analysis), a slight tendency toward an increase in percentage motility was observed when GSH is added (Gadea et al., 2005b).

\section{Conception Rate}

The age of semen had no influence on 60 to 90 day non- return rate. It was noted that bulls with the greatest decline in forward motility during the freezing process had the lowest fertility rates; where as decline in forward motility during the storage period apparently did not influence fertility (Gemeinhardt, 1969).

Lundgren (1980) observed lower non-return rate with frozen semen stored for longer period as compared to recently frozen semen.

Chauhan et al. (1983) found the conception rate to first insemination to be $51.1 \%$ and overall conception rate with 2.5 services, having no significant differences between the years of preservation. 
Bukowinski and Kucharski (1989) observed in four artificial insemination stations and found the 60 day non return rate to first insemination to be $74.0,74.5,72.7$ and $73.0 \%$ with the corresponding calving rates of $72.0,67.9,71.4$ and $55.4 \%$ respectively.

Konstandoglo (1989) found a lower conception rate to first insemination with cross bred bull semen having spermatozoa with chromosomal abnormality.

Chenoweth (1990) evaluated a significant correlation between conception rate with scrotal circumference, sperm motility and the proportion of sperm abnormalities as $0.58,0.47$ and 0.40 respectively.

Semen having less than $50 \%$ post thaw motility was not correlated with fertility and ascribed the cause of reduced non return rate to reduced capability to survive in female genital tract or higher no. of incompetent spermatozoa at the site of fertilization (Soderquist et al., 1991).

Wolf (1996) found in young and old bulls that the 90 day non-return rates $(\mathrm{P}<0.05)$ in cows inseminated with frozen thawed semen to be $56.9 \%$ and $51.2 \%$ respectively.

Mostari et al. (2005) investigated dairy performance among various seasons through semen quality and 30 day non return rate and evaluated that the season had no significant effect on sperm fertility and sperm motility of diluted semen. The significant $(\mathrm{P}<0.01)$ bull to season interaction was noticed in semen volume, initial sperm motility, post thaw percentages, $\mathrm{P}^{\mathrm{H}}$ and fertility but not on sperm motility of diluted semen.

There was no significant difference in pregnancy rates at 60 days after AI between the extenders containing egg yolk (66.7\%, 20/30 animals) or BSA (65.5\%, 19/29 animals). Furthermore, there was no significant difference in the lambing rates $(66.7 \%$ and $62.1 \%)$ and prolificacy (1.25 and 1.56) between the two semen extenders. This indicates that a semi-defined semen extender containing $10 \%$ BSA produces fertility after intrauterine AI that is similar to that achieved with semen extender containing egg yolk. Fertility rate of bulls showed negative correlation $(\mathrm{p}<0.05)$ with sperm abnormality percent $(\mathrm{p}=0.93)$ (Mandal et al., 2007).

The bull wise first insemination conception rate for semen frozen with tris extender were 44.40, 61.90, 47.30 and 57.80 \% respectively and corresponding values for Biociphos plus extended semen were 66.7, 55.047 .6 and $42.8 \%$ for four bulls Viz, HF-555, HF-1473, HF- 1477 and HF1492 respectively (Patil et al., 2008). 


\section{MATERIALS AND METHODS}

The present study was under taken in the Department of Animal Reproduction, Gynaecology and Obstetrics, Orissa Veterinary College, Bhubaneswar, in collaboration with the Frozen Semen Bank, Cuttack during the period of July'07 to July'08. The experiment envisaged use of two semen additives viz. reduced glutathione and cysteine hydrochloride in routine semen extender used for cryo-preservation. The efficacy of such applications was evaluated on the basis of their seminal traits and conception rate following artificial breeding.

\section{SOURCE OF ANIMALS}

Three good freezable and three poor freezable Jersey Crossbred bulls belonging to Frozen Semen Bank, Cuttack under Animal Husbandry \& Veterinary Services, Government of Orissa were selected for this study. The semen from these bulls was routinely used for Artificial Insemination following collection and ultra low freezing. The bulls were within age group of 4 to 6 years and were maintained under optimum nutrition and managemental practices as per the standard criteria fixed for maintenance of breeding bulls in bull stations.

\section{COLLECTION OF SEMEN}

The semen was collected at homosexual mount by artificial vagina (AV) twice a week as described by Williams (1920). A total of 36 ejaculates@6 from each bull were collected for assessment and preservation. The semen so collected was examined microscopically and all routine seminal traits were recorded. The samples were then processed and finally preserved in Liquid Nitrogen. Post freeze seminal traits were also recorded after usual thawing.

\section{ROUTINE SEMEN EVALUATION}

\section{Colour}

Colour of semen was determined by visual inspection and was recorded immediately after collection. Any abnormal colored semen sample other than white and yellow colour was discarded.

\section{Volume}

The ejaculated volume was noted directly from the graduated semen collection tube and recorded in milli liter. 


\section{Mass Activity}

Soon after collection of semen, a small thick drop of undiluted semen was taken on a preheated clean glass slide and examined under microscope with biotherm arrangement $\left(37^{\circ} \mathrm{C}\right)$. Mass activity of neat semen was graded with a scale "O" to +++ as described by Bhosrekar ( 1990).

\section{Individual Motility}

The individual motility of the semen sample was examined under microscope by standard procedure described by ZemJanis (1970).

\section{Sperm Concentration}

The sperm concentration was measured using photo electric colorimeter described by Paulenz et al. (1995).

After warming up the equipment, $6.0 \mathrm{ml}$ sodium citrate solution $(2.9 \%)$ was taken in a cuvette and the equipment was set to $100 \%$ transmittance. To this $0.1 \mathrm{ml}$ Neat semen was added and gently mixed. The cuvette was placed in side the bovine photometer and reading ( $\%$ transmission / optical density) was recorded. This reading was translated into sperm concentration with the help of a standard graph prepared by haemocytometer technique.

The percentage of transmission recorded by the photometer had to be translated to the sperm concentration. For this the percentage transmission had to be recorded for a large number of semen samples of different sperm concentrations. The actual sperm concentration of the samples was estimated by the conventional haemocytometer method. This gave a fundamental relationship between the percentage of transmission and sperm concentration. Based on this relationship a working table showing the percentage of transmission and the corresponding sperm concentration could be worked out using graphic or mathematical technique.

\section{Procedure}

$1.6 \mathrm{ml}$ distilled water containing $2.96 \%$ sodium citrate was taken in the cuvette, the cuvette was inserted into the photo electric meter and the refractive index was adjusted to $100 \%$.If it did not show $100 \%$ it was adjusted by using knob. Unless 4 and 5 were fulfilled the instrument was not used for evaluation of semen concentration. The optical density of the semen was recorded and using the ready reckoner chart, sperm concentration was assessed and recorded. 


\section{Live Sperm Percent}

Live and dead count of spermatozoa was done by Eosin - Nigrosin stain as described by Yavuz (2001).

\section{Assessment of Sperm Plasma Membrane Permeability}

Assessment of sperm plasma membrane permeability was described by Bruno and Jacques (1996). Propidium Iodide (PI), which binds to and stains cellular DNA, is impermeable to the plasma membrane (Garner and Johnson, 1995).

PI has previously been used to assess membrane integrity in mammalian spermatozoa and has been shown not to stain intact spermatozoa. The number of spermatozoa permeable to PI among highly motile sperm populations was scored.

\section{Procedure}

Stock solution $(10 \mu \mathrm{l})$ of PI $\left(0.5 \mathrm{mg} / \mathrm{ml}\right.$ in PBS, stored in the dark at $\left.-20^{\circ} \mathrm{C}\right)$ was added to 1 $\mathrm{ml}$ of the sperm preparation. Then $10 \mu \mathrm{l}$ of the resulting suspension was placed between slide and cover slip for observation under both phase contrast and epi-fluorescent microscopy. This simultaneous observation was obtained by maintaining the light intensity at a sufficiently high level to score non -fluorescent and fluorescent spermatozoa under phase contrast microscopy and sufficiently low to visualize the red fluorescence of PI- permeant spermatozoa. Percentages of spermatozoa permeant to PI were estimated from at least 200 spermatozoa per preparation.

\section{Live and dead assessment of sperm by plasma membrane permeability}

The procedure was followed as described by Selvaraju et al. (2007) and live and dead percentage of sperm was recorded.

\section{Procedure}

Fluorogenic staining was carried out by incubating $80 \mu 1$ of semen with $100 \mu$ of phosphate buffered saline (PBS) based CFDA/PI staining solutions. The stock and staining solutions were kept frozen until used. CFDA/PI staining solutions contained $0.8 \mu \mathrm{l}$ of CFDA stock solution (4 $\mathrm{mg} / \mathrm{ml}$ in DMSO) and $5 \mu \mathrm{l}$ of PI stock solution $(0.27 \mathrm{mg} / \mathrm{ml}$ in PBS). The semen samples were incubated at $37{ }^{\circ} \mathrm{C}$ for $15 \mathrm{~min}$. After incubation, $10 \mu \mathrm{l}$ of $0.2 \%$ glutaraldehyde was added to the solution and 4-5 $\mu \mathrm{l}$ of the stained sample was placed on warm slide, a cover slip was applied, and the preparations were examined under magnifications of $\times 400$ using an HBO 50 mercury lamp illuminated, epic-fluorescence microscope (Nikon eclipse 50i) equipped with CFDA filter set 
(excitation filter, $510-560 \mathrm{~nm}$; emission filter, $505 \mathrm{~nm}$ ). A minimum of 200 sperms of each frozen thawed sample were observed. The sperm showing complete green fluorescence were considered plasmalemma intact (live), and the cells showing partial or complete red nuclei were classified as dead.

\section{Abnormal Sperm Morphology study}

Abnormal morphology of spermatozoa was studied by examination of wet smear and dry smear. Wet smear were prepared by suspending semen in formal saline solution (Hancock, 1952) and dry smears were stained with Rose Bengal stain (Herman and Madden, 1953). Percentage of abnormal sperm was recorded.

\section{Study of Acrosomal integrity Status}

\section{Fluorogenic Staining (FITC-PSA)}

Fluorogenic staining (FITC-PSA) for acrosomal integrity of sperm was done as was described by Selvaraju et al. (2007).

\section{Procedure}

Semen samples were thawed at $37^{\circ} \mathrm{C}$ for 35 seconds in warm water bath, washed with $\mathrm{BO}$ media containing $0.6 \%$ BSA (0.6 gm in $100 \mathrm{ml}$ distilled water) and centrifuged at $2000 \mathrm{rpm}$ for $5 \mathrm{~min} .0 .5 \mathrm{ml}$ samples at the bottom was left and mixed slowly and thoroughly. Mixed samples were thoroughly smeared on a grease free slide. Smears were fixed with methanol for 10 minutes and washed three times with DPBS (Dulbecco's modified Phosphate Buffered Saline) media containing BSA. $50 \mu \mathrm{l}$ of freshly prepared final working staining solution (FITC-PSA, $5 \mu \mathrm{g} / \mathrm{ml}$ and PI $7 \mu \mathrm{g}$ / $\mathrm{ml}$ ) was put on to the slides (Fluoresein isothiocyanate-Pisum Sativum Agglutinin, stock- 1mg/ml with Propidium iodide, stock $0.27 \mathrm{mg} / \mathrm{ml}$ ) and incubated the slides in the dark for 25 min, washed three times with DPBS and were dried in a dark room. A drop of $80 \%$ glycerol with DPBS (8ML glycerol $+2 \mathrm{ml}$ DPBS) was put over the slide and covered with cover slip and sealed tightly. The preparations were examined under magnifications of $\times 400$ using an HBO 50 mercury lamp illuminated, epifluorescence microscope (Nikon eclipse 50i) equipped with CFDA filter set (excitation filter, 510-560 nm; emission filter, $505 \mathrm{~nm}$ ). A minimum of 200 sperms of each frozen thawed sample were observed.

The sperm showing complete green fluorescence were considered plasma lemma intact (live), and the cells showing partial or complete red nuclei were classified as dead. 


\section{Incubation Study}

The incubation study of the semen samples were conducted as per method described by Kumaresan et al. (2005). Randomly selected straws from treatment and control groups were thawed at $37^{\circ} \mathrm{C}$ for 30 seconds and the semen was pooled and washed twice by centrifugation in protein free sperm-TALP ( $\mathrm{pH}$ 7.3). Then the sperm pellet was dissolved in incubation media i.e. sperm TALP containing $6 \mathrm{mg} / \mathrm{ml}$ of bovine serum albumin (BSA) and incubated for $4 \mathrm{~h}$ at $37^{\circ} \mathrm{C}$. The motility, viability and acrosomal integrity were assessed at hourly interval to find out the effect of Cysteine and Glutathione on spermatozoan characteristics during incubation.

\section{Fluorogenic assessment of Mitochondrial Membrane Potential}

Several fluorescent probes have been used to evaluate sperm mitochondrial function. Mitochondrial probes are actively transported into actively respiring mitochondria, therefore, the more active the mitochondrial respiration, the more probe is accumulated. Rhodamine 123 was initially used to evaluate sperm mitochondrial function (Graham et al., 1990; Evenson et al., 1982), but can only differentiate between respiring and non-respiring mitochondria. More recently, JC-1 has been used to assess spermatozoal mitochondria function (Garner et al., 1997; Thomas et al., 1998).

At low concentrations, JC-1 remains in the monomeric state and fluoresces green. However, at high concentrations, JC-1 forms aggregates that fluoresce orange. Therefore, JC-1 has not only the ability to distinguish functional from non-functional mitochondrial, but permits different levels of mitochondrial function to be determined by intensity of mitochondrial 'orangeness'. In support of sperm motility being a measure of mitochondrial function, the percentages of sperm with functioning mitochondria is highly correlated to sperm motility, regardless of whether rhodamine 123 (Evenson et al.,1982; Auger et al.,1989) or JC-1 (Garner et al.,1997; Thomas et al.,1998) is used to evaluate mitochondrial function.

Mitochondrial membrane potential was assessed by using JC-1 (5, 5', 6, 6'-tetrachloro-1, 1', 3, 3'- tetraethylbenzimidazolylcarbocyanine iodide) as per Selvaraju et al.(2007).

\section{Procedure}

Three micro liters of a prepared solution (1.53mM JC-1 in dimethyl sulfoxide) was added to a $100 \mu \mathrm{l}$ sample of frozen thawed sperm $(80 \times 106 / \mathrm{ml})$, and incubated for $30 \mathrm{~min}$ at $37 \circ \mathrm{C}$ and counterstaining of nuclei was performed with $10 \mu \mathrm{l}$ of PI stock solution $(0.27 \mathrm{mg} / \mathrm{ml})$. Then, cells were washed once in PBS and resuspended in PBS, smeared, and analyzed with an HBO 50 
mercury lamp illuminated, epifluorescence microscope (Nikon Eclipse 50i) equipped with CFDA filter set. A minimum of 200 sperms were observed at $\times 400$ magnifications. The cells with yellowish to orange fluorescence (aggregates) in the mid piece were considered as cells having mitochondrial membrane potential.

\section{Lipid Peroxidation Assay (LPO)}

Lipid peroxidation level of sperm and seminal plasma was measured by determining the malonaldehyde (MDA) production, using thiobarbituric acid (TBA) as per the method of Buege and Aust (1978) and modified by Suleiman et al. (1996).

\section{Procedure}

The straws were cut and the semen was centrifuged at $3000 \mathrm{rpm}$ for $15 \mathrm{~min}$ and the seminal plasma was removed. Then the sperm pellet was resuspended in $2 \mathrm{ml}$ of PBS ( $\mathrm{pH} 7.2$ ) or a variable volume to obtain a sperm concentration of $20 \times 10^{6} / \mathrm{ml}$. Lipid peroxide levels were measured in spermatozoa after the addition of $2 \mathrm{ml}$ of TBA-TCA reagent $(15 \% \mathrm{w} / \mathrm{v}$ TCA, $0.375 \% \mathrm{w} / \mathrm{v}$ TBA and $0.25 \mathrm{~N} \mathrm{HCL}$ ) to $1 \mathrm{ml}$ of sperm suspension. The mixture was treated in a boiling water bath for 1 hour. After cooling, the suspension was centrifuged at 3000rpm for $10 \mathrm{~min}$. The supernatant was then separated, and absorbance was measured at $535 \mathrm{~nm}$. The MDA concentration was determined by the specific absorbance coefficient $\left(1.56 \times 10^{5} \mu \mathrm{mol} / \mathrm{cm}^{3}\right)$.

$$
\begin{gathered}
\text { MDA produced }(\mu \mathrm{mol} / \mathrm{ml})=\frac{\mathrm{OD} \times 10^{6} \times \text { Total volume }(3 \mathrm{ml})}{1.56 \times 10^{5} \times \text { Test volume }(1 \mathrm{ml})} \\
=\frac{\mathrm{OD} \times 30}{1.56}
\end{gathered}
$$

\section{Feulgen's Staining Technique for Examination of DNA}

\section{A. $\quad 10$ percent neutral buffered -formalin solution}

Sodium phosphate, monobasic $\left(\mathrm{NaH}_{2} \mathrm{PO}_{4}\right) \quad 4 \mathrm{~g}$

Sodium phosphate, dibasic $\left(\mathrm{Na}_{2} \mathrm{HPO}_{4}\right) \quad 6.5 \mathrm{~g}$

Formaldehyde (37 to 40 percent) $\quad 100 \mathrm{ml}$

Distilled water $\quad 900 \mathrm{ml}$

B. Sulfate water

10 percent (w/v) Potassium Metabi-sulfite $\left(\mathrm{K}_{2} \mathrm{O}_{5} \mathrm{~S}_{2}\right) \quad 5 \mathrm{ml}$ 
This should be prepared fresh.

\section{Procedure}

The smear was prepared in neat semen and allowed it to air dry for at least 1 hour, fixed in 10 percent neutral buffered formal saline for 15 minutes and washed in running water for 10 minutes. The smear was hydrolyzed in $5 \mathrm{~N}$ HCL for 30 minutes, washed in running water for 5 minutes and dipped in Schiff's reagent for 30 minutes. The slide were rinsed in sulfate water for 2 minutes and repeated for three times. They were washed in running water for 10 minutes and dried in air, and examined at x 1000 under phase- contrast microscope.

\section{Cervical Mucus Penetration Test (CMPT)}

The action of estrogen that is being produced by the developing follicle increases the amount of mucus, improves its elasticity (Spinnbarkait test) and causes the micelles within the mucus to straighten and allow the passage of the spermatozoa .There is positive correlation.

\section{Collection of cervical mucus}

Cervical mucus was collected from estrous cows using a plastic insemination sheath as per Dabas and Maurya (1988) and evaluated for sperm receptivity (Pattabiraman et al., 1967).

After manipulation of the uterus and cervix per rectum to extrude mucus into the vagina, the mucus is aspirated from anterior vagina from the estrous cow using a sterilized plastic insemination sheath connected to a $20 \mathrm{ml}$ syringe and the mucus was transferred immediately to the screw capped vials or test tube and transported to the laboratory.

\section{Evaluation of mucus samples}

The collected mucus was evaluated for the following characters

1. Spinbarket test: Should have good elasticity to be selected.

2. Ferning (Fern pattern): Mucus showing typical fern like crystallization should be selected.

3. White side test: A white side test using 5\% sodium hydroxide should be done to evaluate the mucus for the infection. $1 \mathrm{ml}$ of mucus should be heated with $1 \mathrm{ml}$ of $5 \%$ sodium hydroxide upto the boiling point and the sample which developed yellow colour should be considered positive for 
mild endometritis and should not be used for test. Only the samples show clear colour should be selected.

4. Sperm Receptivity: A small portion of mucus and drop of semen should be put in a glass slide under a cover slip and incubated for $15-30$ minutes at $37^{\circ} \mathrm{C}$. Mucus receptivity to the spermatozoa is assessed by the formation of phalanges in mucus. Mucus samples with inadequate migration should be discarded.

\section{Procedure}

\section{Capillary tube technique}

Non-heparinzed hemtocrite capillary tube $80 \mathrm{~mm}$ length and $0.8 \mathrm{~mm}$ diameter is utilized for the test. To aspirate the mucus into the capillary tube loading manifold should be prepared from a scalp vein set and $5 \mathrm{ml}$ syringe. The needle from one end of the scalp vein set was removed and the capillary tube should attach. The other end was attached to $5 \mathrm{ml}$ syringe. The mucus sample is aspirated in to the attached end of the capillary tube by drawing the plunger of the syringe. Trilling mucus was cut with scissors, leaving a small amount protruding from the filled end of the tube. The capillary tube was then removed from the loading manifold, China clay /PVC powder was used to plug one end of the tube and the tube is allowed to stand for 5 minutes at $38^{\circ} \mathrm{C}$. If the mucus contracts during the period the mucus column was adjusted by plugging more clay.

A glass slide was graduated by scoring millimeter marks along side using a diamond pen. A sperm reservoir (cuvette of photometer can be used) in then glued on to the graduated slide. The reservoir was filled with the semen under test; one end of the capillary tube with the mucus protruding slightly was placed in the semen and the tube laid between the supports on the slide. The system was now laid horizontally in a Petridis together with some moistened filter paper to proved humidity and incubated at $37^{\circ} \mathrm{C}$ for one hour. The capillary tube was now examined under X400 magnification phase control microscopic and the distance traveled by the sperm up the tube was ascertained. This distance traveled by the vanguard of the sperm was measured in milliliters (Anil Kumar and Devanathan, 1995).

The average sperm progression speed (SPS) through the mucus column was calculated in micron /second (Gaddum Rosse et al., 1980). Difference among bulls and individual mucus samples were tested by factorial experiment in randomized block design. 


\section{SEMEN PRESERVATION}

Semen collected from 6 bulls (Three Good freezable and three poor freezable) were preserved at $-196^{\circ} \mathrm{C}$ in Liquid Nitrogen.

Extenders containing different additives with tris as a base were assigned to three groups

Group I: Egg Yolk Tris Glycerol (EYTG) extender as control.

Group II: EYTG extender with addition of Cysteine @ 5 mM as additives.

Group III: EYTG extender with Glutathione@ 5 mM as additives.

\section{PREPARATION OF STANDARD EYTG DILUENT}

The standard protocol for preparation of EYTG was followed and fresh extender is prepared on daily basis just before collection in the morning hour.

\section{Preparation of standard tris diluent}

Tris Buffer

Citric Acid

Fructose

Triple Distilled Water
$-2.42 \mathrm{gm}$.

$-1.36 \mathrm{gm}$.

$-1.0 \mathrm{gm}$.

$-73.6 \mathrm{ml}$.

The mixture was autoclaved at 5 PSI pressure for 20 minutes, cooled and kept in refrigerator at $4^{0} \mathrm{C}$. The following ingredients were added to the freshly prepared Tris diluent and mixed for 30 minutes with the help of a magnetic stirrer.

$\begin{array}{ll}\text { Egg Yolk } & -20.0 \mathrm{ml} \\ \text { Glycerol } & -6.4 \mathrm{ml} \\ \left.\text { Antibiotic (Ambistrin }{ }^{\circledR}\right) & -0.1 \mathrm{gm} \\ \text { Antibiotic (Benzyl Penicillin) } & -1.0 \text { lakh units }\end{array}$

\section{PREPARATION OF STRAWS}

Breed, Bull No., Date of collection and Center of production were printed on straws by automatic printing machine. Straws of different colour were used for different extenders.

Group I: Red straws for standard EYTG diluent (control) 
Group II: White straws for standard EYTG diluents plus Cysteine Hydro chloride extender

GroupIII: Orange straws for standard EYTG diluents plus Glutathione extender.

\section{POST THAW SEMEN EVALUATION}

Randomly selected straws were taken from different groups and thawed for 30 seconds in a water bath maintained at $37^{\circ} \mathrm{C}$. Following thawing of semen straws, several semen evaluation tests will conducted to determine the quality of semen.

\section{POST THAW MOTILITY}

The post thaw motility was assessed as per method described by Amelar et al. (1980).

\section{POST THAW HYPO-OSMOTIC SWELLING TEST}

Hypo-Osmotic Swelling Test (HOST) of spermatozoa, as an in-vitro fertility test, was conducted to evaluate the semen sample in the procedure as described by Ravell and Morde (1994).

\section{Preparation of HOST solution}

\section{$\underline{\text { Ingredients }}$}

Fructose

Tri Sodium Citrate

Distilled Water (up to)

Osmolarity $\left(\mathrm{mOsm} \mathrm{kg}^{-1}\right)$

\section{$\underline{\text { Raw Semen Test }}$}

$13.51 \mathrm{gm}$

$7.35 \mathrm{gm}$

$1000 \mathrm{ml}$

\section{Procedure}

5 to 10 micro liter of semen was mixed with $1 \mathrm{ml}$ of test solution after being thawed at $37^{0} \mathrm{C}$ for 30 seconds. Semen with test solution in small vial or test tube was incubated for 1 to 2 hours at $35^{\circ} \mathrm{C}$.After incubation the mixture was well mixed and a small drop was put on a pre warmed microscopic slide and covered with a cover slip. The slide was examined on a warm stage at $400 \mathrm{X}$ magnification under phase contrast optics. A total 200 sperm are counted in at least 5 different fields. A drop is placed in the counting chamber and 200 sperm are counted in 5 squares. The 
proportion of sperm cells exhibiting hypo osmotic swollen positive response was expressed as percent (Correa et al., 1997).

The percentage of swollen sperm was calculated by the following formula

$$
=\frac{\text { Number of sperm swollen }}{\text { Total number of sperm counted }} \times 100
$$

\section{COMPUTER ASSISTED SEMEN ANALYSIS (CASA)}

Analysis of the motion parameters were determined using a CASA system (Sperm Class Analyzer, Microptic, Barcelona, Spain) at 0, $1.00 \mathrm{hr}, 2.00 \mathrm{hrs}$ and $4.00 \mathrm{hrs}$ following thawing the semen sample. Analysis was described by Joaqu et al., 2007 and was based on the examination of twenty-five consecutive digitalized images per second using a $10 \mathrm{X}$ negative phase contrast objective (Nikon, Eclipse E200) and fitted with stage warmer (Linkam DC 60). The CASA-derived motility characteristics studied were total motility (\%), progressive motility (\%), curvilinear velocity (VCL in $\mu \mathrm{m} / \mathrm{s}$ is the average path velocity of the spermatozoa head along its actual trajectory ), straight-line velocity (VSL, um/s VSL in $\mu \mathrm{m} / \mathrm{s}$ is the average path velocity of the spermatozoa head along a straight line from its first to last position), average path velocity (VAP, $\mathrm{um} / \mathrm{s}$ ), linearity of the curvilinear trajectory ( $\mathrm{LIN},(\%)$ ). LIN is the ratio between VSL and VCL), straightness (STR, ratio of VSL/VAP, (\%)), wobble of the curvilinear trajectory (WOB, ratio of $\mathrm{VAP} / \mathrm{VCL}, \%$ ), amplitude of lateral head displacement (ALH in $\mu \mathrm{m} / \mathrm{s}$ is the average value of the extreme side-to-side movement of the spermatozoa head in each beat cycle.) and beat crossfrequency $(\mathrm{BCF}, \mathrm{Hz})$. The parameters set were frame rate: $60 \mathrm{~Hz}$ frame acquired: 25, minimum contrast: 30 , cell size (range 5 to 70 microns $^{2}$ ), spermatozoa concentration ( 5 to $8 \times 10^{6} / \mathrm{ml}$ ), and motile cells $\left(>10 \mu \mathrm{m} / \mathrm{s}\right.$ at $\left.37^{\circ} \mathrm{C}\right)$, progressive forward motility ( $>80 \%$ straightness index, STR); circular movement $(<50 \%$ linearity index, LIN).

Motility analysis was carried out in $5 \mu$ of semen samples $(0.25$ mldiluted semen in $1.75 \mathrm{ml}$ of Tris buffer) placed onto a pre-warmed $\left(37^{\circ} \mathrm{C}\right)$ microscopic slide covered with the $18 \times 18 \mathrm{~mm}^{2}$ cover slip. A minimum of 200 spermatozoa from at least two different drops of each sample were analyzed from each specimen. The number of objects incorrectly identified as spermatozoa were manually removed and final analysis was done for each sample.

\section{INSEMINATION}

Out of six collections from three good freezable and three poor freezable bulls, a total number of 2010 French mini straws were prepared, which were subdivided into two experimental 
groups and one untreated control group. About 250 straws were utilized for conducting different motility studies including CASA and other staining and biochemical studies. The rest of the semen straws were dispatched to Teaching Veterinary Clinical Complex, Orissa Veterinary College, Bhubaneswar and Veterinary Dispensary of different centers like Sahid Nagar, Nayapalli and Konark. Inseminations were carried out in healthy, normally cycling heifers and cows by qualified veterinarian.

\section{CONCEPTION RATE}

The fertility of the experimental groups were (straws contained additives) assessed on the basis of actual pregnancy diagnosis between 55-60 days post insemination.

The conception rate was calculated for different groups are as follows.

Conception rate $(\%)=\frac{\text { Number of cows conceivedinirstinse } \min \text { tion }}{\text { Number of } A I} \times 100$

\section{DATA ANALYSIS}

Data obtained was subjected to statistical analysis as per standard methods described by Snedecor and Cochran (1967). 


\section{RESULTS}

In the present investigation three Good and three Poor freezable crossbred Jersey bulls belonging to Frozen Semen Bank, Cuttack, Government of Orissa were selected and six ejaculates from each bull diluted with Tris extender with out additives was considered as Control (Group I) incorporation of additives either reduced Glutathione (Group III) or Cysteine hydrochloride (Group II) as semen additive before cryo preservation at $-196^{\circ} \mathrm{C}$ in liquid nitrogen was also taken up. Routine semen evaluation was carried out prior to extension of semen without additives. Various seminal characteristics like volume, mass activity and individual motility, live sperm count, sperm abnormalities and acrosomal damage were investigated. Some of the parameters were also assessed consequent to post freezing. Besides this, specific in-vitro test like HOST and CMPT were conducted in post freezing semen samples. The efficacy of semen additives like Cysteine hydrochloride and Glutathione were assessed on the basis of seminal traits and the resultant conception rate following artificial insemination. The data generated were presented in tabular form.

\section{SEMINAL ATTRIBUTES OF GOOD AND POOR FREEZABLE CROSSBRED BULLS}

The routine semen analysis and certain in-vitro fertility tests were undertaken from the semen samples collected from three good and three poor freezable crossbred bulls. The result of 18 ejaculates obtained from good freezable bulls and 18 ejaculates obtained from poor freezable bulls were presented in table-I. It was observed that the volume ( $\mathrm{ml}$ ),mass activity (grade),individual motility (percent),sperm concentration $\left(\mathrm{x}^{6} 0^{6} / \mathrm{ml}\right)$, live sperm (percent),total sperm abnormality (percent),loss of acrosomal integrity (percent) and hypo-osmotic swelling positive sperm (percent) were estimated to be $6.4 \pm 0.41,2.5 \pm 0.13,77.13 \pm 0.60,1240.26 \pm 82.77,89.95 \pm 0.49,6.33 \pm 0.19$, $6.46 \pm 0.9$ and $85.78 \pm 0.42$ respectively in semen samples of good freezable crossbred bulls. Similarly for the poor freezable bull semen the values were found to be $5.2 \pm 0.36,2.4 \pm 0.13$, $75.33 \pm 0.77,1007.53 \pm 50.28,85.22 \pm 0.28,9.39 \pm 0.27,12.73 \pm 1.12$ and $80.38 \pm 0.32$ respectively (Table-1). The test of significance revealed that a highly significant $(\mathrm{p}<0.01)$ difference exists between good and poor freezable bull semen with respect to individual motility, percent of live sperm, total sperm abnormality, loss of acrosomal integrity and hypo-osmotic swelling positive spermatozoa. Similarly a significant $(\mathrm{p}<0.05)$ difference was found between volume and sperm concentration. 


\section{POST THAW SEMEN EVALUATION}

\section{Motility}

The semen after initial evaluation, divided into three groups i.e., group I (control), Group II (cysteine hydrochloride) and group III (glutathione) were estimated for certain seminal attributes, in-vitro tests, biochemical tests and sperm functional tests. It was revealed from the present investigation that in good freezable bull semen the motility was highest in group III (74.12 \pm 0.39$)$ and lowest in group II $(61.83 \pm 0.48)$ and in group I, it was $(62.08 \pm 0.39)$. The motility (percent) in poor freezable bulls showed the highest in group III $(66.83 \pm 0.42)$ followed by group II $(59.06 \pm$ $0.82)$ and group I $(54.32 \pm 0.24)$. Analysis of variance has revealed that a high significant difference $(\mathrm{p}<0.01)$ exists among various experimental groups irrespective of good or poor freezable crossbred bulls under study (Table 2).

Test of significance has revealed that there was no significant difference among good freezable crossbred bulls between group I and group II, but both the values were significantly different $(p<0.01)$ from group III. In poor freezable crossbred bulls group I differed significantly $(p<0.05)$ from group II, where as the motility value for group III has registered a highly significant difference $(\mathrm{p}<0.01)$ from group I and group II.

\section{Livability}

The total live sperm (per cent) in good freezable and poor freezable bull semen were estimated to be $63.09 \pm 0.37$ and $56.78 \pm 0.56$ in control (group I) where as in group II it was estimated to be $70.85 \pm 0.32$ and $60.92 \pm 0.89$ while it was $76.28 \pm 0.37$ and $69.32 \pm 0.35$, respectively in group III. A high significant difference $(\mathrm{p}<0.01)$ was also found between the treatment groups of both good and poor freezable semen with respect to the total live sperm per cent.

As regards to livability, each group differed significantly $(\mathrm{p}<0.01)$ from each other. In poor freezing group the livability percent behaved similar to their good freezer counter parts exhibiting a highly significant difference between various combinations (Table 2).

\section{Total sperm abnormality}

The total abnormal sperm percent in good freezable bull semen were estimated to be $(9.45 \pm$ 0.94) when semen was diluted after incorporation with glutathione (Group III), whereas it was higher in group II $(11.42 \pm 0.74)$ and highest $(13.18 \pm 1.41)$ in the control group where there was no incorporation of such additives (group-I). Similar trends were observed in poor freezable semen 
which was $14.26 \pm 0.18,15.87 \pm 0.26$ and $18.06 \pm 0.18$ in group III, II and I respectively. Analysis of variance revealed a highly significant difference $(\mathrm{p}<0.01)$ in both the groups of good and poor freezable bull semen with respect to total sperm abnormality (Table 2).

The total sperm abnormality percent for group I was significantly different from $(\mathrm{p}<0.05)$ from group III, whereas rest of combination did not reveal any significant difference in good freezing group. Contrastingly the values for sperm abnormality from group I, II and group III differs significantly $(\mathrm{p}<0.01)$ from each other in poor freezable group.

\section{Loss of acrosomal integrity}

The Loss of acrosomal integrity percent of sperm were estimated and values for Good freezable (group I, II and III) and Poor freezable crossbred Jersey bulls (group I, II and III) were recorded as $12.20 \pm 0.71,10.96 \pm 0.92 \& 8.44 \pm 0.63$ and $15.97 \pm 0.5,15.02 \pm 0.7 \& 13.01 \pm 0.60$ per cent, respectively. Comparison of the values recorded for loss of acrosomal integrity of sperms in three different groups had registered highly significant difference $(\mathrm{p}<0.01)$ (Table 3$)$.

Loss of acrosomal integrity in good freezing bulls, the group I value recorded a highly significant difference $(\mathrm{p}<0.01)$ from group III, whereas it was not significantly different from group II. The group II value differed significantly $(\mathrm{p}<0.05)$ from group III.

In poor freezing group the group I and group II has revealed no significant difference for the same parameters. But the loss of acrosomal integrity was lowest for group III and it was significantly different $(\mathrm{p}<0.01)$ from other groups.

\section{Hypo-Osmotic Swelling Test (HOST)}

Hypo-osmotic swelling positive sperm (per cent) in good freezable and poor freezable bull semen were estimate to be $65.74 \pm 0.27$ and $57.03 \pm 0.322$ in control (group I). Whereas in group II it was estimated to be $70.47 \pm 0.35$ and $57.65 \pm 0.47$, while it was $81.19 \pm 0.3$ and $71.29 \pm 0.26$ in group III respectively. A high significant difference $(\mathrm{p}<0.01)$ was also found to exist between the treatment groups of both good and poor freezable semen with respect to the Hypo-osmotic swelling positive sperm (Table 3).

The HOST values were significantly different $(\mathrm{p}<0.01)$ between various experimental groups in good freezable crossbred bulls. The HOST value for group: III revealed a highly significant difference $(p<0.01)$ both from group I or group II in poor freezable crossbred bull semen. 


\section{Cervical mucus penetration test (CMPT)}

Estimation of vanguard distance traveled by the Sperm in the cervical mucus with respect to the post thaw semen samples in the control, cysteine and glutathione group is shown in Table 3 . In group III (glutathione), group II (cysteine) and group I (control) of good freezer, the vanguard distance in the cervical mucus were recorded $(\mathrm{mm})$ to be $31.16 \pm 1.09,27.76 \pm 0.98$ and $25.84 \pm$ 1.35 respectively. Analysis of variance between the treatment groups registered a highly significant difference $(\mathrm{P}<0.01)$.

The corresponding value with regard to vanguard distance in the cervical mucus in poor freezable bulls were recorded as $25.44 \pm 1.35,23.35 \pm 1.18$ and $20.24 \pm 1.09 \mathrm{~mm}$, respectively. The difference between these three treatment groups has showed a high significant difference $(\mathrm{P}<0.01)$ (Table 3).

The CMPT value for group III in good freezing cross bred bulls was significantly different $(p<0.05)$ from group II and the difference was highly significant against group I. In poor freezing crossbred bulls semen the CMPT value for group III revealed a difference from group I $(\mathrm{p}<0.01)$ and from group II $(\mathrm{p}<0.05)$, but there was no significant difference between group I and group II.

\section{DNA fragmentation}

Determination of DNA Fragmentation of sperm with respect to the post thaw semen samples in the control, cysteine and glutathione group is shown in Table-4. In good freezer, the DNA Fragmentation of Sperm were recorded to be $10.77 \pm 0.67,8.83 \pm 1.13$ and $6.94 \pm 1.28$ per cent in groups I, group II and group III respectively. Analysis of variance between the treatment groups with good freezable bull has registered a high significant difference $(\mathrm{P}<0.01)$.

Similarly the corresponding value with regard to DNA Fragmentation of Sperm in poor freezable crossbred bull was recorded as $24.70 \pm 1.16,16.42 \pm 2.19$ and $9.98 \pm 1.17$ per cent respectively. The analysis of variance between these three treatment groups of poor freezable crossbred bull showed a high significant difference $(\mathrm{P}<0.01)$.

The DNA fragmentation value of group II showed no significant difference with group I or group III, whereas group I differed significantly from group III $(\mathrm{p}<0.05)$ in good freezer bulls. Comparison for DNA fragmentation value for group I against either group II or group III revealed a highly significant difference $(p<0.01)$, whereas group II value differed significantly $(p<0.05)$ from group III (Table 4) in poor freezable bulls. 


\section{Lipid Peroxidation (LPO)}

Measurement of Lipid Peroxide (Malondialdehyde) with respect to the post thaw semen samples in the group I, group II and group III were shown in Table 4. In good freezer Malondialdehyde production (Lipid Peroxide) were recorded (nmol/10 ${ }^{8}$ sperm) to be $2.73 \pm 0.85$, $2.60 \pm 0.76$ and $2.17 \pm 0.82$ in group I, group II , and group III, respectively.

The corresponding value with regard to Malondialdehyde (Lipid Peroxide) in semen of poor freezable crossbred bulls was recorded as 3.68 \pm 1.65 , 3.50 1.42 and $3.17 \pm 1.34 \mathrm{nmol} / 10^{8}$ sperm respectively. Analysis of variance between the treatment groups revealed that there was no significant difference among groups both in good and poor freezable bulls.

\section{Mitochondrial Membrane Potential (MMP)}

The Mitochondrial membrane potential (per cent) in good freezable and poor freezable bull semen were estimated to be $26.11 \pm 3.27$ and $25.93 \pm 1.60$ in control (group I) where as in group II it was estimated to be $28.63 \pm 3.08$ and $26.11 \pm 2.96$. While it was $29.78 \pm 0.98$ and $27.55 \pm 1.64$ in group III respectively. Analysis of variance between the treatment groups had revealed that there was no significant difference among groups both in good and poor freezable bulls (Table 4).

\section{Incubation test in pooled semen samples}

The pooled samples of the frozen and processed semen from good and from poor freezable crossbred bulls were put to incubation test at $0,1 \mathrm{hr}, 2 \mathrm{hrs}$ and $4 \mathrm{hrs}$ of incubation at $37^{\circ} \mathrm{C}$. Instead of routine microscopic motility study, computer assisted semen analysis was employed to study forward progressive motility percent, non-progressive motility, total motility and static sperms and all were expressed in percent. The forward progressive motility ranged between $56.71 \pm 2.42$ (poor freezer) and $67.07 \pm 1.67 \mathrm{in}$ good freezer. The progressive forward motility of $67.07 \pm 1.67$ recorded at $0 \mathrm{hrs}$ was minimized to $42.84 \pm 4.30$ after $4 \mathrm{hr}$ incubation. Similarly the poor freezer recorded a forward progressive motility percent of $56.71 \pm 2.42$ at $0 \mathrm{hr}$ which gradually minimized to $28.55 \pm$ 3.63 at 4 hrs of incubation. In all the cases, the difference in forward progressive motility per cent between the good and poor freezer was found to be significant $(\mathrm{p}<0.05)$ except for at $1 \mathrm{hr}$ incubation where the difference was highly significant $(\mathrm{p}<0.01)$ (Table-5).

The non- progressive motility in good freezer at different incubation point from 0 to $4 \mathrm{hrs}$, ranged between $17.74 \pm 1.89$ and $13.97 \pm 2.47$ while the value for the poor freezer ranged similarly between $21.71 \pm 2.15$ and $25.25 \pm 3.11$ at all the hrs of incubation which was recorded to be highly significant at $(\mathrm{p}<0.01)$ level. 
The total motility percent recorded in good freezer dropped down from $84.80 \pm 1.87$ at $0 \mathrm{hrs}$ to $60.93 \pm 3.53$ at $4 \mathrm{hrs}$ of incubation. Similarly the poor freezer has recorded the values between $78.41 \pm 2.75$ and $50.05 \pm 3.80$. At all the hrs of incubation the difference between poor and good freezer inn total motility percent were recorded non significant except at 4 hrs of incubation which was significant at $(\mathrm{p}<0.05)$ level.

The static sperm percent recorded for the good freezer as $15.20 \pm 1.87$ at $0 \mathrm{hrs}$ was maximized to $39.07 \pm 3.53$ at $4 \mathrm{hrs}$ incubation. The corresponding value for poor freezer was recorded as $21.59 \pm 2.75$ at $0 \mathrm{hrs}$ which was maximized to $49.95 \pm 3.80$ at $4 \mathrm{hrs}$ incubation period.

The difference in static sperm percent between good and poor freezer were found to be non significant in all the hrs of incubation except at $4 \mathrm{hrs}$ incubation where it was highly significant $(\mathrm{p}<$ 0.01 ) (Table 5).

\section{Comparison of Motility between Control and Treatment Groups at different hours of Incubation}

The Progressive Forward motility, Non-progressive motility, Total motility and Static sperms at $0 \mathrm{hr}$ incubation of the semen treated with cysteine were $62.40 \pm 41.80,19.23 \pm 28.75$, $81.63 \pm 27.63$ and $18.37 \pm 27.63$ percent respectively and the corresponding values of the glutathione and control groups were $61.70 \pm 3.06,20.18 \pm 3.14,81.88 \pm 3.94 \& 18.12 \pm 3.94$ and $61.57 \pm 31.50$, $19.75 \pm 19.49,81.32 \pm 30.78$ and $18.68 \pm 30.78$ respectively. Analysis of variance between the treatment groups in all the motility parameters was not significant at $0 \mathrm{hr}$ incubation (Table 6).

The Progressive Forward motility, Non-progressive motility, Total motility and Static sperms at $1 \mathrm{hr}$ incubation of the semen treated with cysteine were $55.29 \pm 6.87,17.88 \pm 3.71,73.16$ \pm 6.89 and $26.84 \pm 6.89$ percent respectively and the corresponding value of the glutathione and control groups were $61.25 \pm 6.71,22.54 \pm 3.94,83.67 \pm 3.07 \& 16.33 \pm 3.07$ and $61.14 \pm 4.58,18.42 \pm$ $5.00,79.67 \pm 4.85$ and $20.33 \pm 4.85$ respectively. Analysis of variance in between the treatment groups indicated a highly significant difference in the forward progressive, non- progressive and total motility and not significant in the static at $1 \mathrm{hr}$ incubation.

The Progressive Forward motility, Non-progressive motility, Total motility and Static sperms at $2 \mathrm{hrs}$ incubation of the semen treated with cysteine were 47.88 $\pm 3.76,18.91 \pm 4.60$, $66.79 \pm 6.31$ and $33.21 \pm 6.31$ percent respectively and the corresponding value of the glutathione and control groups were 50.81 $\pm 5.83,19.09 \pm 4.40,69.90 \pm 2.43$ and $30.10 \pm 2.43$ and 48.32 \pm 4.03 , $17.78 \pm 1.86,66.10 \pm 3.10$ and $33.90 \pm 3.10$ respectively. Analysis of variance in between the treatment 
groups indicated not a significant difference in the forward progressive, non- progressive, total motility and the static sperms at $2 \mathrm{hr}$ incubation.

The Progressive Forward motility, Non-progressive motility, Total motility and Static sperms at $4 \mathrm{hrs}$ incubation of the semen treated with cysteine were $36.36 \pm 4.20,18.03 \pm 3.72$, $54.39 \pm 4.26$ and $45.61 \pm 4.26$ percent respectively and the corresponding value of the glutathione and control groups were 36.68 $\pm 7.30,20.61 \pm 3.07,57.29 \pm 5.65 \& 42.71 \pm 5.65$ and 34.03 \pm 5.91 , $20.76 \pm 2.91,54.79 \pm 5.59$ and $45.21 \pm 5.59$ respectively. Analysis of variance in between the treatment groups indicated a no significant difference in the forward progressive, non- progressive, total motility and the static sperms at 4 hrs incubation (Table 6).

\section{Computer Assisted Sperm Analysis (CASA) of velocity parameters of sperm}

Besides the motility parameters such as progressive forward motility, non-progressive motility, total motility and static sperms already studied through CASA during the incubation study, other important parameters like the Curvilinear Velocity [(VCL) ( $\mu \mathrm{m} / \mathrm{sec}$.$) , Straight line$ Velocity $[(\mathrm{VSL})(\mu \mathrm{m} / \mathrm{sec})$.$] , Average path Velocity [(VAP) (\mu \mathrm{m} / \mathrm{sec})$.$] , Linearity[(LIN)$ $(\%)]$,Straightness [(STR) (\%)],Wobble [(WOB) (\%)], Amplitude of Lateral Head [(ALH) $(\mu \mathrm{m})]$ and Beat/Cross Frequency $[(\mathrm{BCF}) \mathrm{Hz}]$ were computed for each samples of good and poor freezable crossbred bull semen in experimental and control groups. The results were presented in Table 7. The Curvilinear Velocity [(VCL) was recorded to be higher $(125.03 \pm 4.33)$ at $0 \mathrm{hr}$ incubation than group II $(112.75 \pm 4.42)$, but lower than group III $(130.76 \pm 3.47)$. Similar trend was also recorded at $2 \mathrm{hrs}$ and $4 \mathrm{hrs}$ but the poor freezable cross bred bulls in the experimental group recorded as VCL of $111.36 \pm 12.78 \mathrm{um} / \mathrm{sec}$. which was showing nearly similar values in group II (110.08 \pm 10.30$)$ and much lower than the corresponding figure of group III $(138.63 \pm 4.10)$ at $0 \mathrm{hr}$ incubation (Table 7).

The Straight line Velocity (VSL) $(\mu \mathrm{m} / \mathrm{sec}$.) of good freezable cross bred bulls at $0 \mathrm{hrs}$ in the control group was recorded to be higher $(93.43 \pm 3.21)$ than both the experimental group II $(76.20 \pm$ 10.37) and group III ( $91.84 \pm 3.39)$ at $0 \mathrm{hrs}$ incubation. Contrastingly at $1 \mathrm{hr}, 2 \mathrm{hrs}$ and $4 \mathrm{hrs}$ of incubation the VSL of good freezable of the experimental group was in-between the corresponding figures of group II and group III, group III having the higher figures. The poor freezable crossbred also behaved similarly recording a figures of $79.72 \pm 11.60$ which was higher than that of group II $(73.04 \pm 8.29)$ and lower than that of group III $(87.23 \pm 11.03)$ at $0 \mathrm{hrs}$ of incubation. For $1 \mathrm{hr}, 2 \mathrm{hrs}$ and $4 \mathrm{hrs}$ of incubation however the poor freezable recorded lower values than that of group II and group III. 
The Straightness (STR) of both good and poor freezable crossbred bulls in all the groups did not differ much at $0 \mathrm{hrs}$ with corresponding figures of group II and group III except for the good freezable of group II recoding a higher value of $84.28 \pm 2.15$ than corresponding figures of group I $(84.09 \pm 3.14)$ and group III $(83.75 \pm 1.67)$.

The Amplitude of Lateral Head displacement (ALH) in recorded with the good freezable of group II (4.01 \pm 0.17$)$ and group III $(4.00 \pm 0.16)$ were higher at $0 \mathrm{hrs}$ of incubation than that of the experimental group $(2.88 \pm 0.03)$. However these differences gradually did not exists at $1 \mathrm{hr}$ and 4 hrs of incubation. The poor freezable of both the experimental groups were similarly recorded higher trend than the experimental group at 2 hrs of incubation. The BCF did not differ much between the groups irrespective of the hrs of incubation (Table 7).

All the differences in different CASA velocity parameters between the treatment groups and control groups were mostly non-significant except for the Curvilinear Velocity (VCL) and Amplitude of Lateral Head displacement (ALH) (Table 7).

\section{Conception rate}

During the period of experiment the conception rate involving 262 inseminations covering both control (79) and experimental groups (cysteine -85 and glutathione -98 ) could be made available. The data were presented in the Table 8.

Among the good freezable highest conception rate of 72 per cent (37/51) was achieved in group III. The control group recorded a conception rate of 51 percent (15/29), while the group II recorded a conception rate of 60 per cent (25/51). A similar picture also was observed with poor freezable crossbred bulls recording a figure of 48 percent (24/50), 56 percent (25/44) and 59 percent (28/47) in group I, group II and group III respectively. The overall conception rate in three different groups was recorded as 49 per cent, 58 per cent and 66 per cent respectively. Comparison of pregnancy value by chi-square analysis did not reveal any significant difference between experimental groups extended with cysteine hydrochloride, glutathione or none.

\section{Correlation among sperm functional parameters and fertility}

The present study revealed that MMP and loss of acrosomal integrity, lipid peroxidation levels, VSL, VCL, VAP, plasmalemma integrity had significantly $(\mathrm{p}<0.05)$ large correlation with fertility. However, post-thaw sperm progressive forward motility had only non-significantly $(\mathrm{p}=$ 0.07) moderate correlation with fertility (Table 9). 
TABLE 1. SEMINAL ATTRIBUTES OF GOOD AND POOR FREEZABLE CROSS BRED BULLS

\begin{tabular}{|c|c|c|c|c|c|c|c|c|}
\hline Good (18) & $\begin{array}{c}6.40 \\
\pm 0.41^{\mathrm{a}} \\
\end{array}$ & $\begin{array}{c}2.50 \pm \\
0.13 \\
\end{array}$ & $\begin{array}{c}77.13 \pm \\
0.60^{\mathrm{a}} \\
\end{array}$ & $\begin{array}{r}1240.26 \\
\pm 82.77^{\mathrm{a}} \\
\end{array}$ & $\begin{array}{r}89.95 \\
\pm 0.49^{\mathrm{a}} \\
\end{array}$ & $\begin{array}{c}6.33 \\
\pm 0.19^{\mathrm{a}} \\
\end{array}$ & $\begin{array}{c}6.46 \\
\pm 0.9^{\mathrm{a}} \\
\end{array}$ & $\begin{array}{c}85.78 \\
\pm 0.42^{\mathrm{a}} \\
\end{array}$ \\
\hline 't' value & $2.17^{*}$ & $0.71^{\mathrm{NS}}$ & $7.54 * *$ & $2.40^{*}$ & $60.01 * *$ & 84. $93 * *$ & $201.77 * *$ & $97.35^{* *}$ \\
\hline
\end{tabular}

NS Non Significant

$* \mathrm{p}<0.05$

$* * \mathrm{p}<0.01$

Figures in parenthesis indicate number of ejaculates 
TABLE 2. POST THAW SPERM MOTILITY, LIVABILITY AND TOTAL ABNORMAL SPERM PERCENT OF GOOD AND POOR FREEZABLE CROSSBRED BULLS IN DIFFERENT DILUENTS

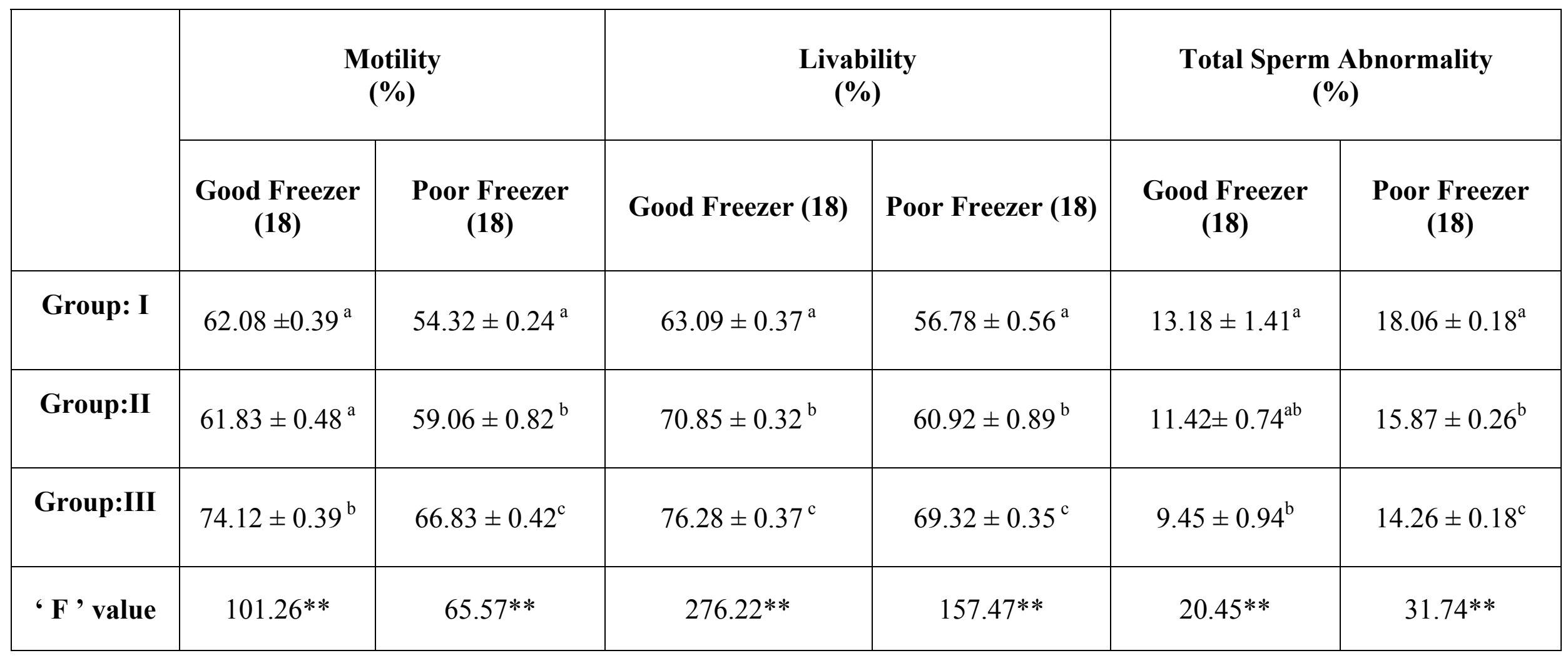

$* * \mathrm{p}<0.01$

Figures with same superscript (a, b and c) do not differ significantly in columns,

Figures in parenthesis indicate number of ejaculates 


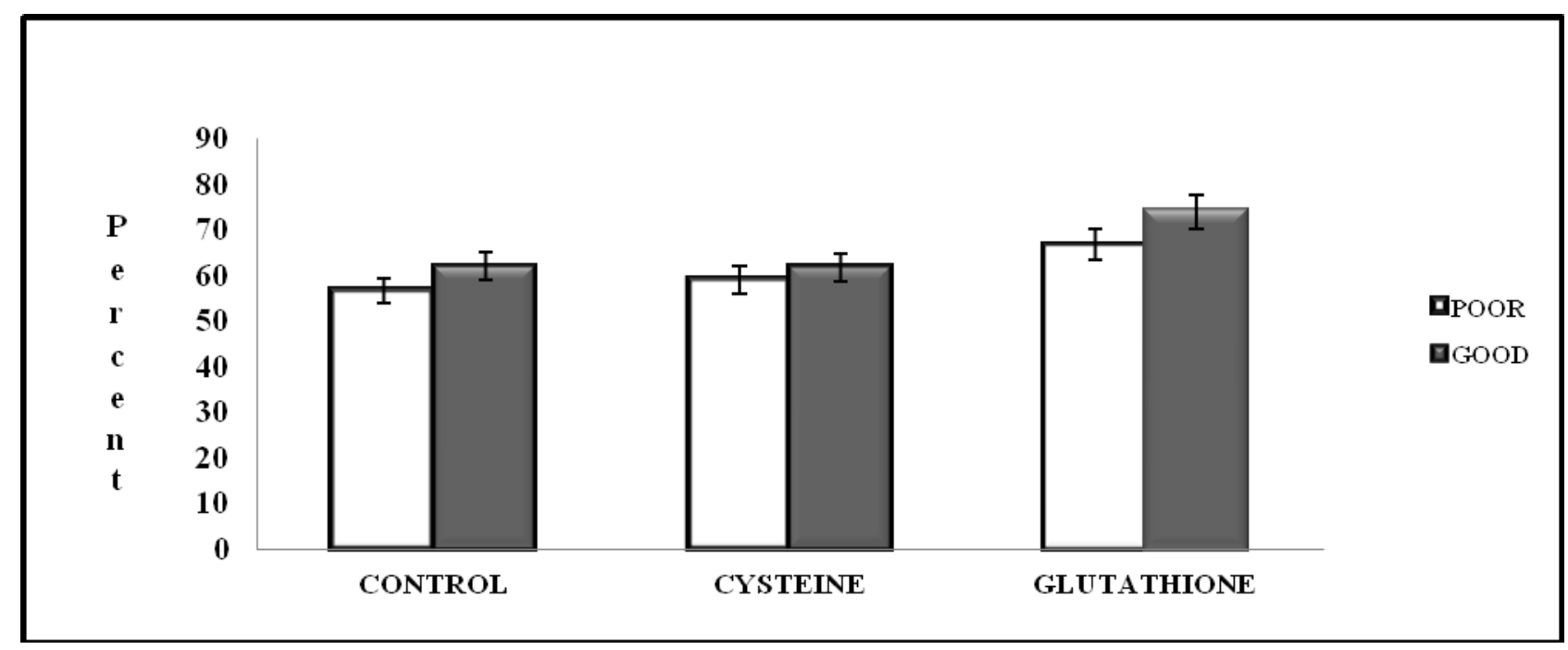

Fig 1. Post thaw sperm motility of good and poor freezable Jersey crossbred bulls in different treatment groups

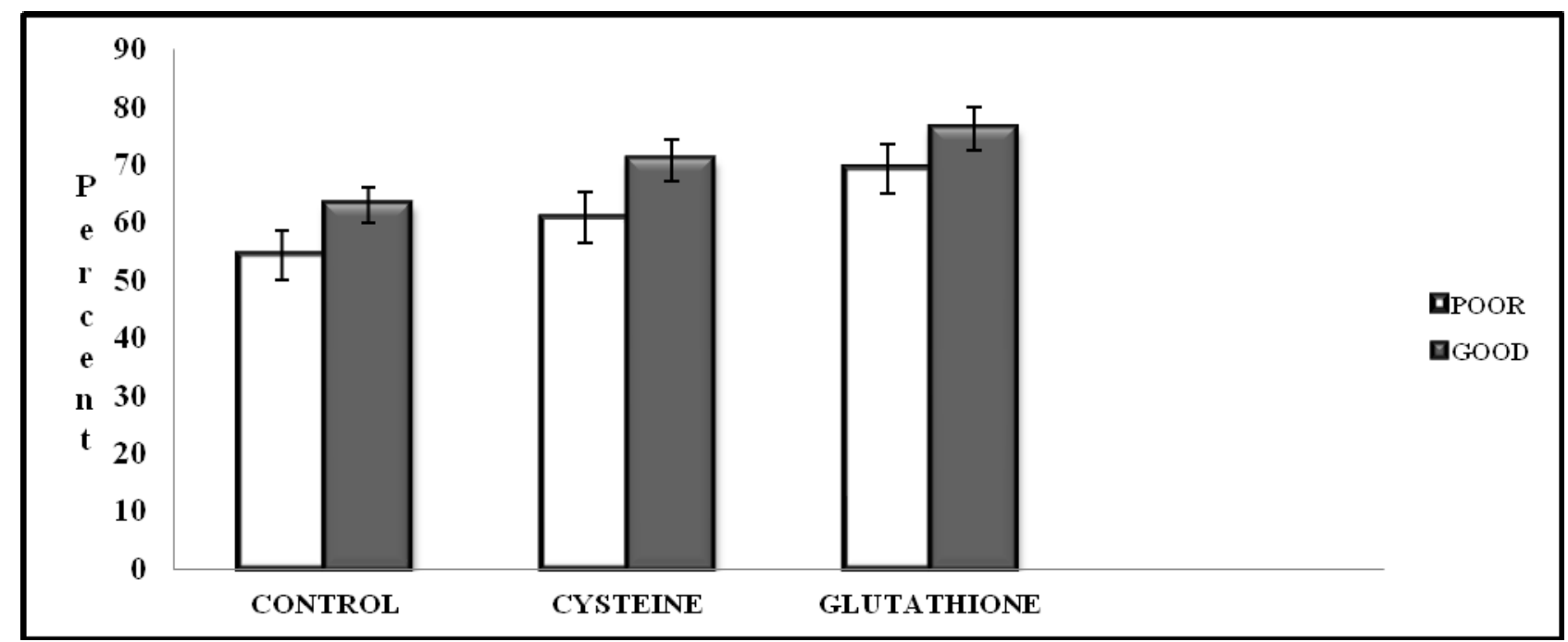

Fig 2. Post thaw sperm livability of good and poor freezable Jersey crossbred bulls in different treatment groups

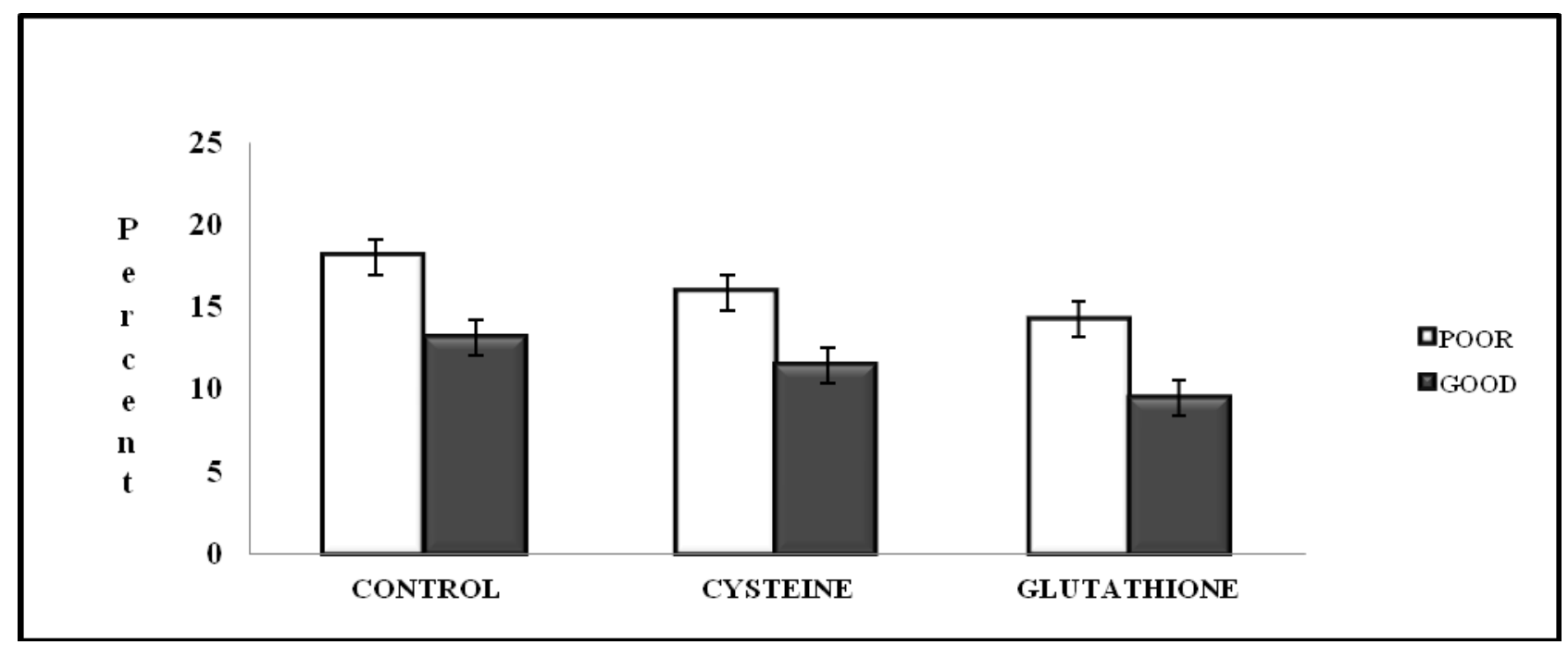

Fig 3. Post thaw total sperm abnormality of semen of good and poor freezable Jersey crossbred bulls in different treatment groups 
TABLE 3. POST THAW LOSS OF ACROSOMAL INTEGRITY, HYPO-OSMOTIC SWOLLEN POSITIVE SPERMS AND CERVICAL MUCUS PENETRATION RATE OF GOOD AND POOR FREEZABLE CROSSBRED BULLS IN DIFFERENT DILUENTS

\begin{tabular}{|c|c|c|c|c|c|c|}
\hline & \multicolumn{2}{|c|}{$\begin{array}{c}\text { Loss of Acrosomal Integrity } \\
(\%)\end{array}$} & \multicolumn{2}{|c|}{$\begin{array}{c}\text { Hypo-Osmotic Swelling Test } \\
\text { (HOST) }(\%)\end{array}$} & \multicolumn{2}{|c|}{$\begin{array}{c}\text { Cervical Mucus Penetration Test } \\
\text { (CMPT) } \\
\text { (mm/hour) }\end{array}$} \\
\hline & $\begin{array}{c}\text { Good Freezer } \\
\text { (18) }\end{array}$ & $\begin{array}{c}\text { Poor Freezer } \\
\text { (18) }\end{array}$ & Good Freezer (18) & Poor Freezer (18) & Good Freezer (18) & $\begin{array}{c}\text { Poor Freezer } \\
\text { (18) }\end{array}$ \\
\hline Group: I & $12.20 \pm 0.71^{\mathrm{a}}$ & $15.97 \pm 0.5^{\mathrm{a}}$ & $65.74 \pm 0.27^{\mathrm{a}}$ & $57.03 \pm 0.32^{\mathrm{a}}$ & $25.84 \pm 1.35^{\mathrm{a}}$ & $20.24 \pm 1.09^{\mathrm{a}}$ \\
\hline Group:II & $10.96 \pm 0.92^{\mathrm{a}}$ & $15.02 \pm 0.7^{\mathrm{a}}$ & $70.47 \pm 0.35^{b}$ & $57.65 \pm 0.47^{\mathrm{a}}$ & $27.76 \pm 0.98^{\mathrm{a}}$ & $23.35 \pm 1.18^{\mathrm{ab}}$ \\
\hline Group:III & $8.44 \pm 0.63^{b}$ & $13.01 \pm 0.6^{\mathrm{b}}$ & $81.19 \pm 0.30^{\mathrm{c}}$ & $71.29 \pm 0.26^{\mathrm{b}}$ & $31.16 \pm 1.09^{b}$ & $25.44 \pm 1.35^{\mathrm{b}}$ \\
\hline ' F', value & $45.42 * *$ & $71.68 * *$ & $255.67 * *$ & $251.00 * *$ & $26.91 * *$ & $28.06^{* *}$ \\
\hline
\end{tabular}

$* * \mathrm{p}<0.01$

Figures with same superscript (a, b and c) do not differ significantly in columns,

Figures in parenthesis indicate number of ejaculates 


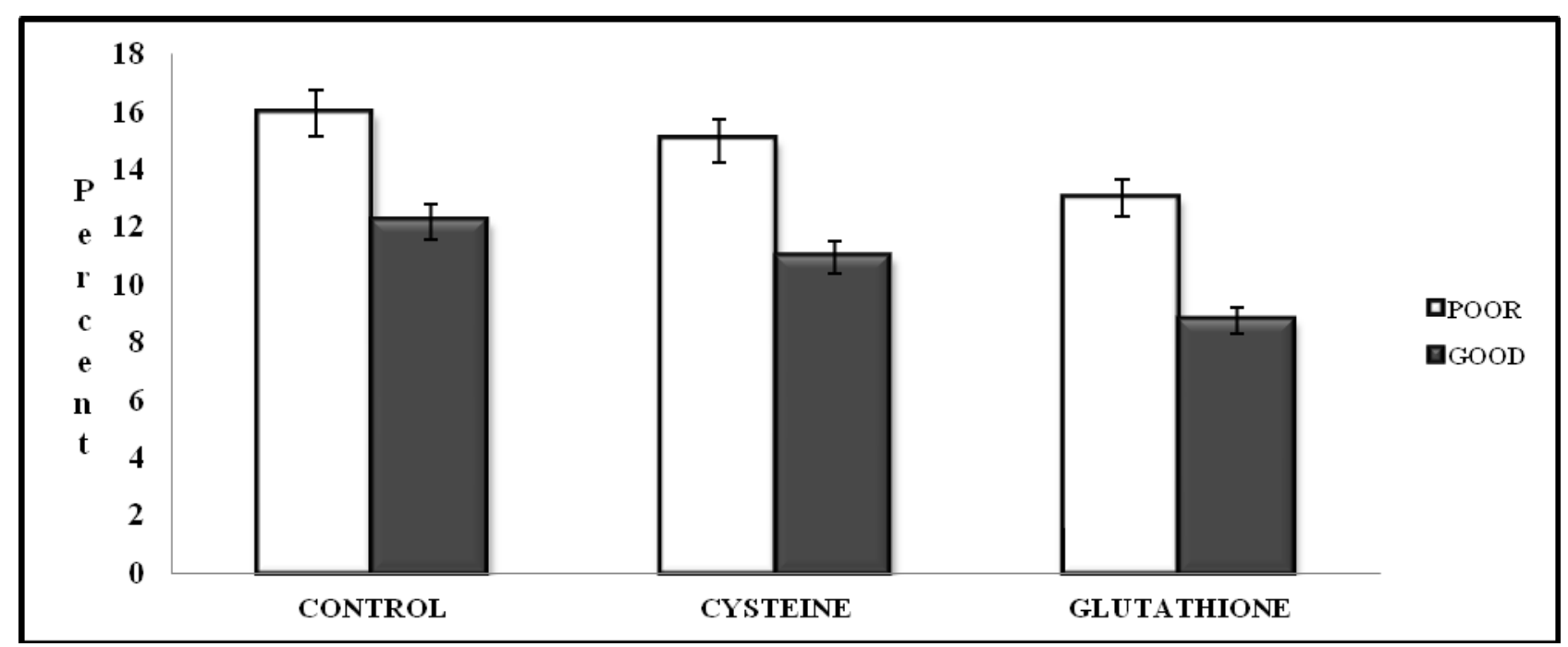

Fig 4. Post thaw loss of acrosomal integrity of good and poor freezable Jersey crossbred bulls in different treatment groups

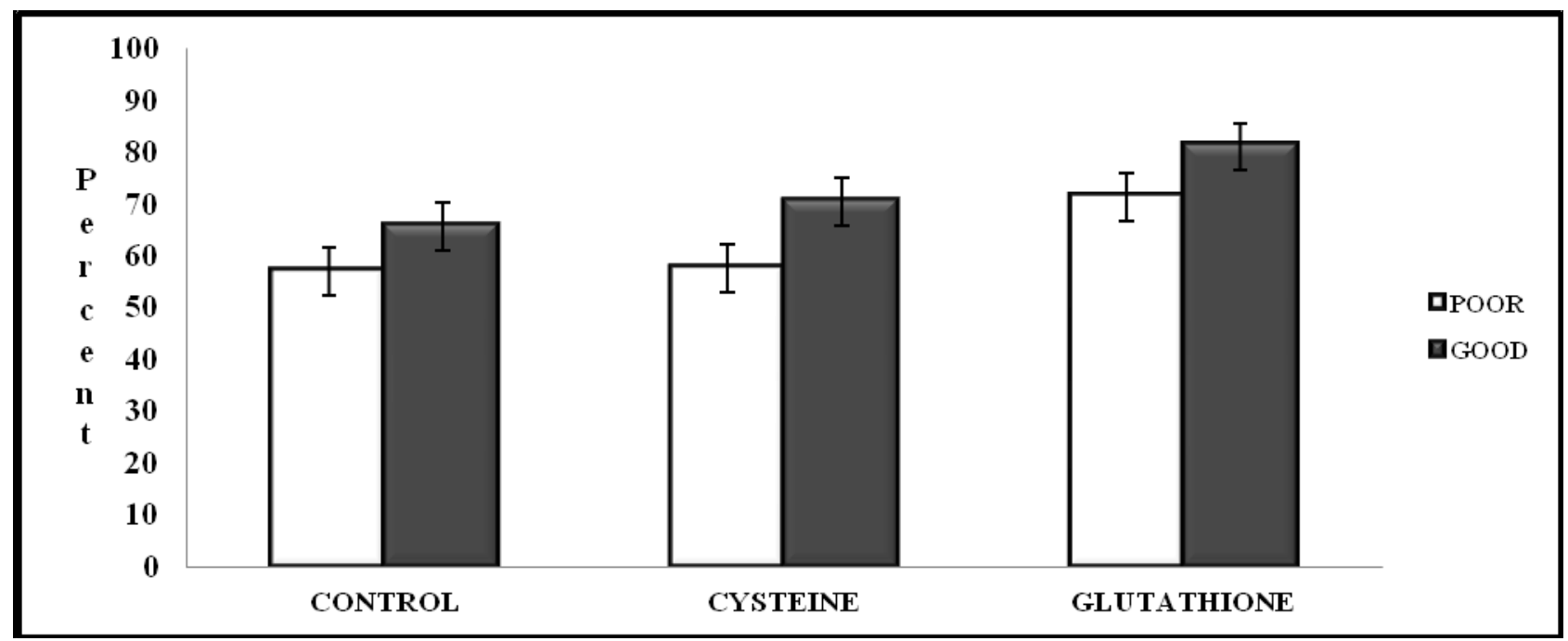

Fig 5. Post thaw HOST of good and poor freezable Jersey crossbred bulls in different treatment groups

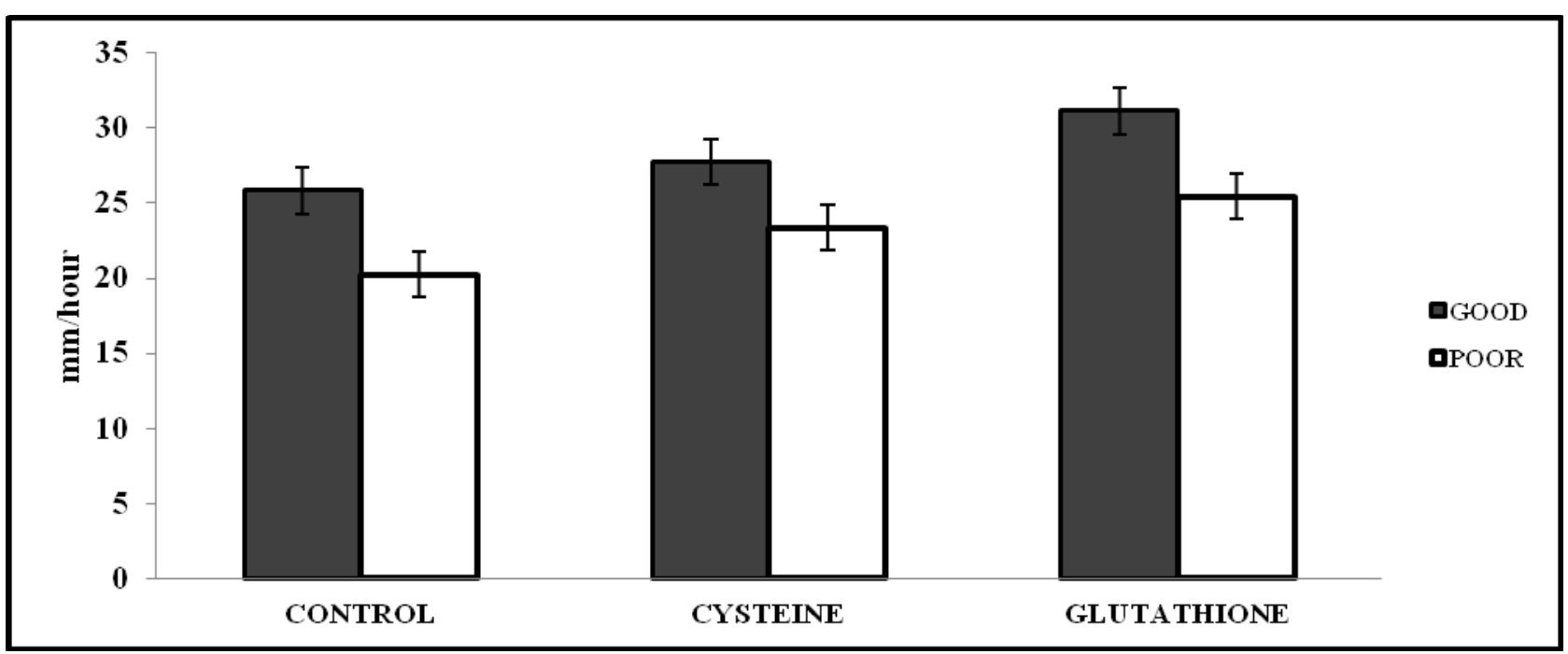

Fig 6. Post thaw vanguard distance traveled by sperm (CMPT) of good and poor freezable Jersey crossbred bulls in different treatment groups 


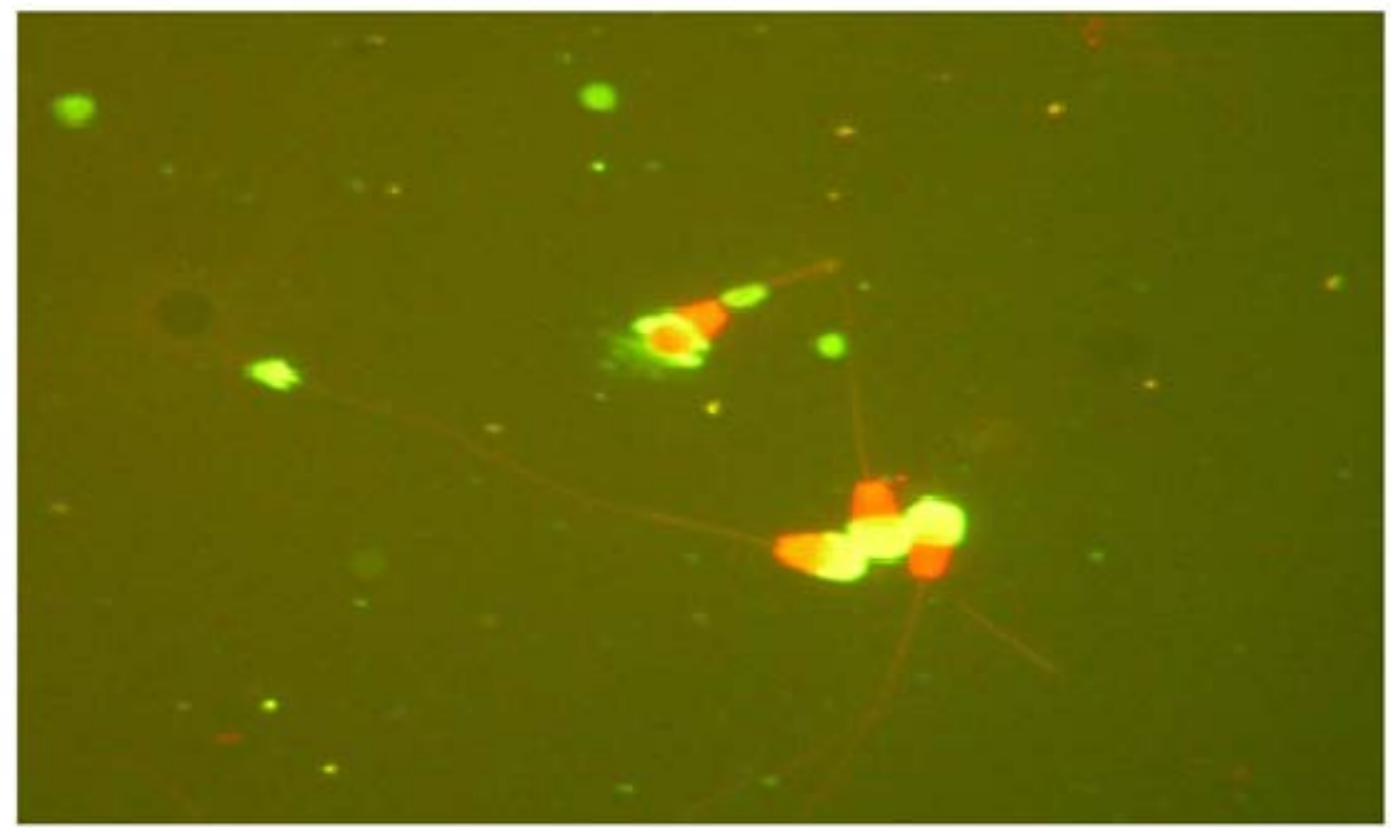

Fig 7. Acrosomal integrity by FITC - PSA Staining

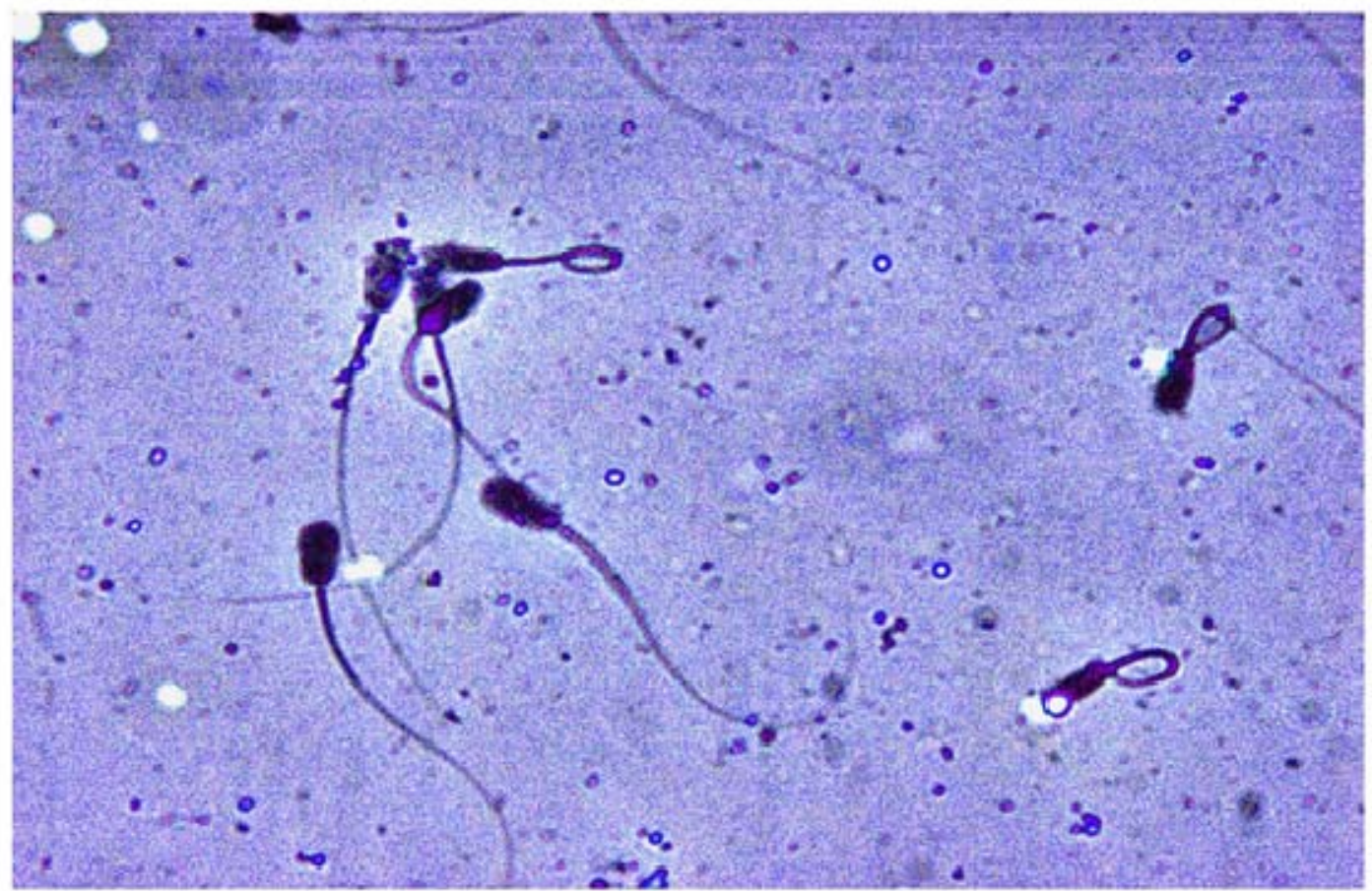

Fig 8. Hypo Osmotic Swelling Test 


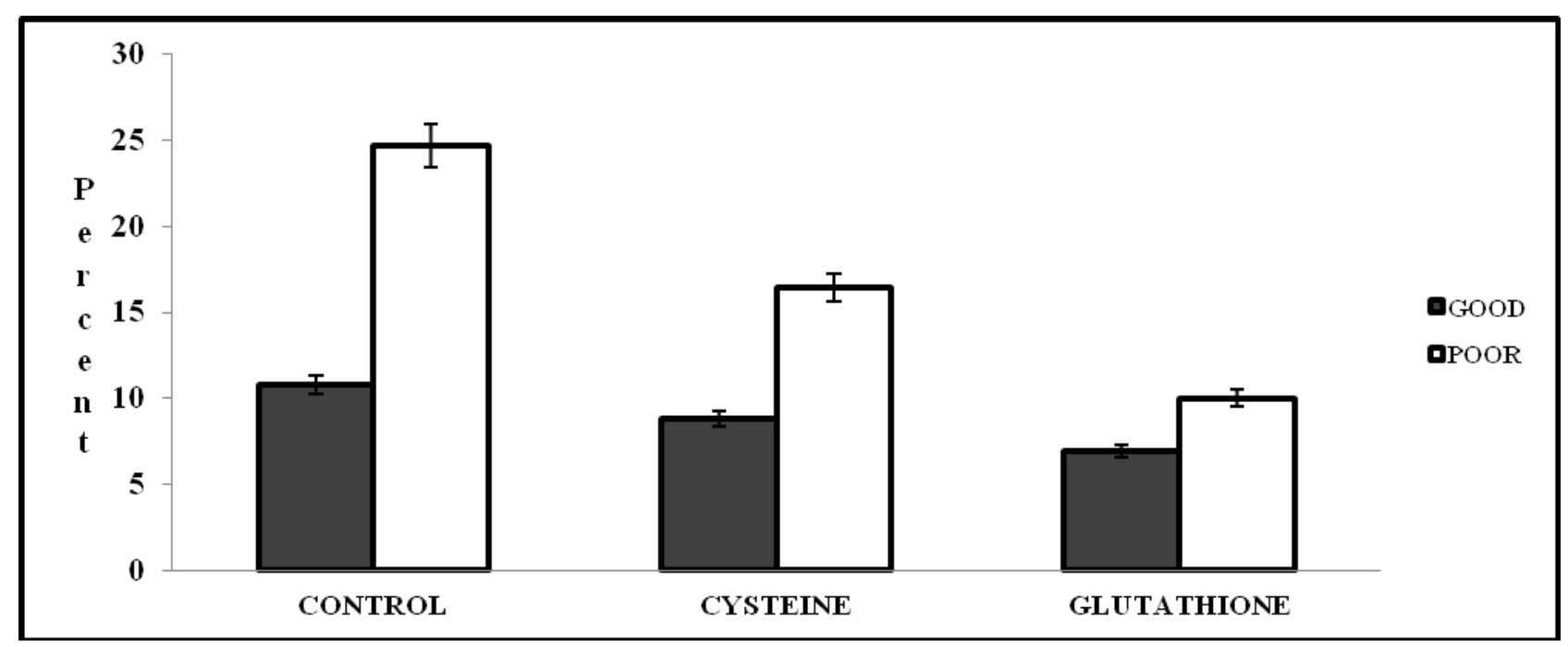

Fig 9. Post thaw DNA fragmentation of sperm of good and poor freezable Jersey crossbred bulls in different treatment groups

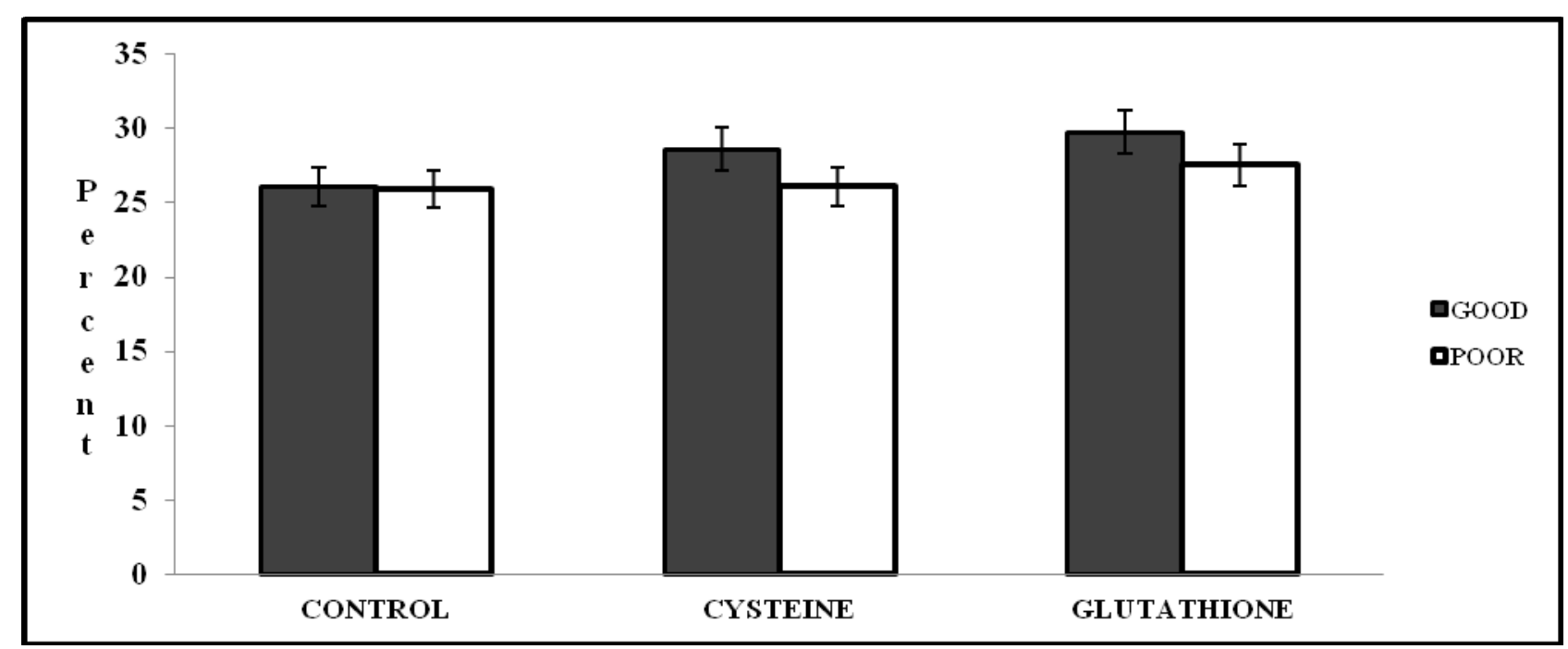

Fig 10. Post thaw MMP of sperm of good and poor freezable Jersey crossbred bulls in different treatment groups

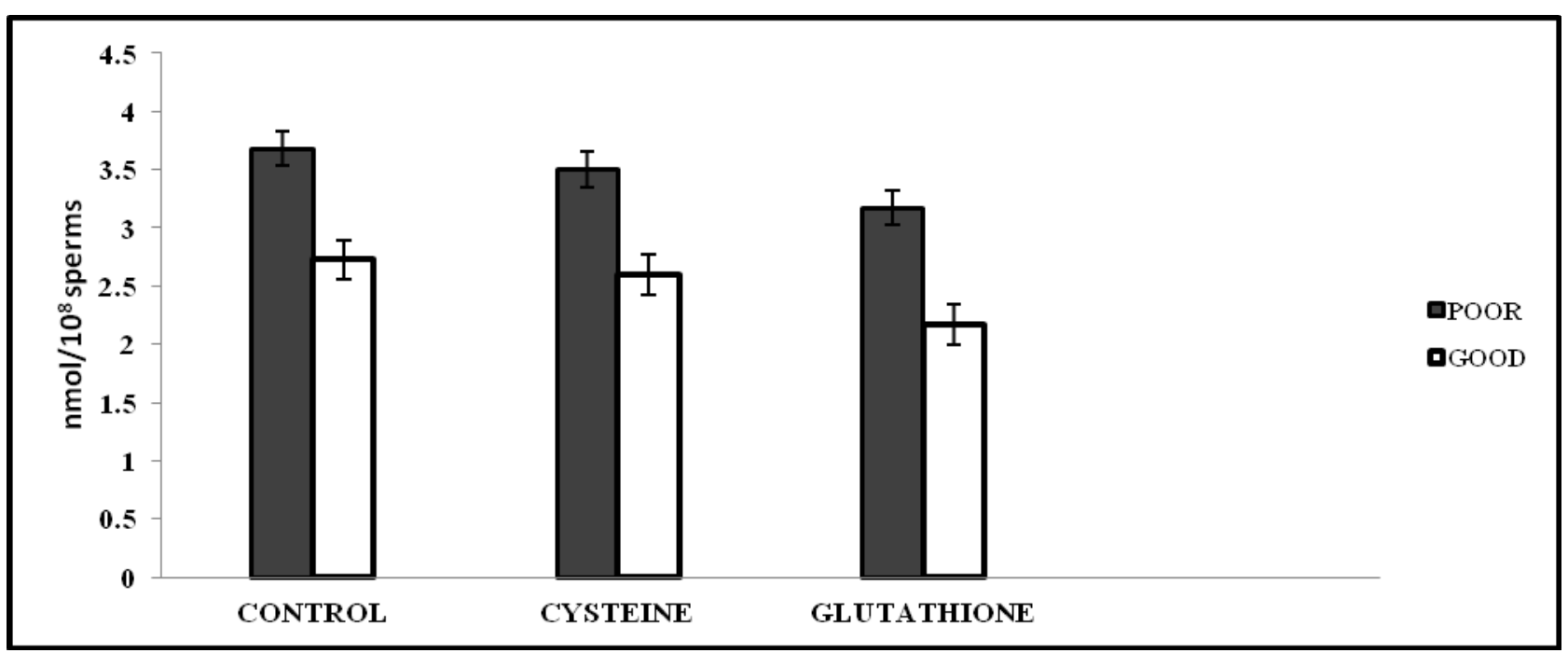

Fig 11. Post thaw (malondialdehyde) LPO of semen of good and poor freezable Jersey crossbred bulls in different treatment groups 


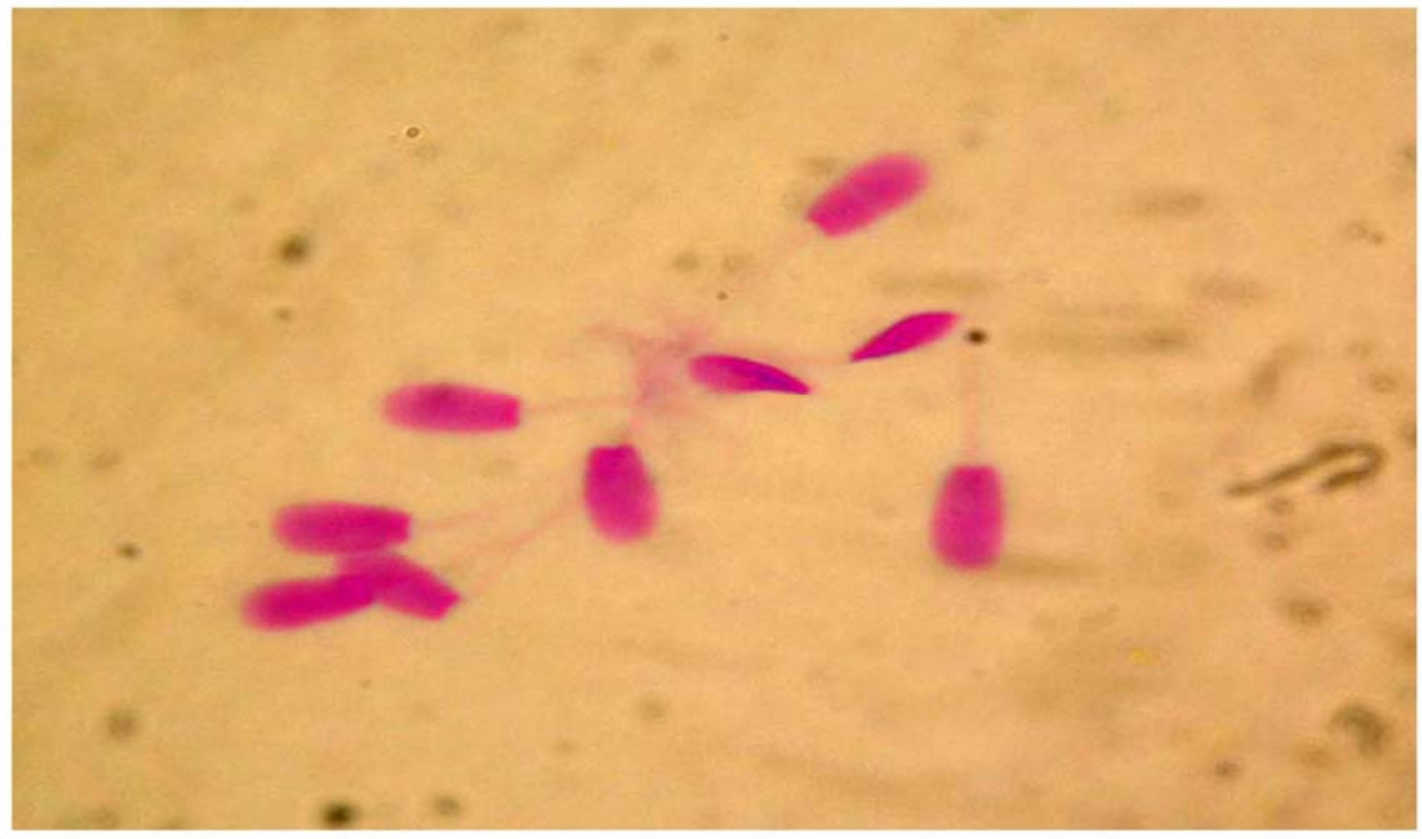

Fig 12. DNA Fragmentation of sperm by Feulgen's staining

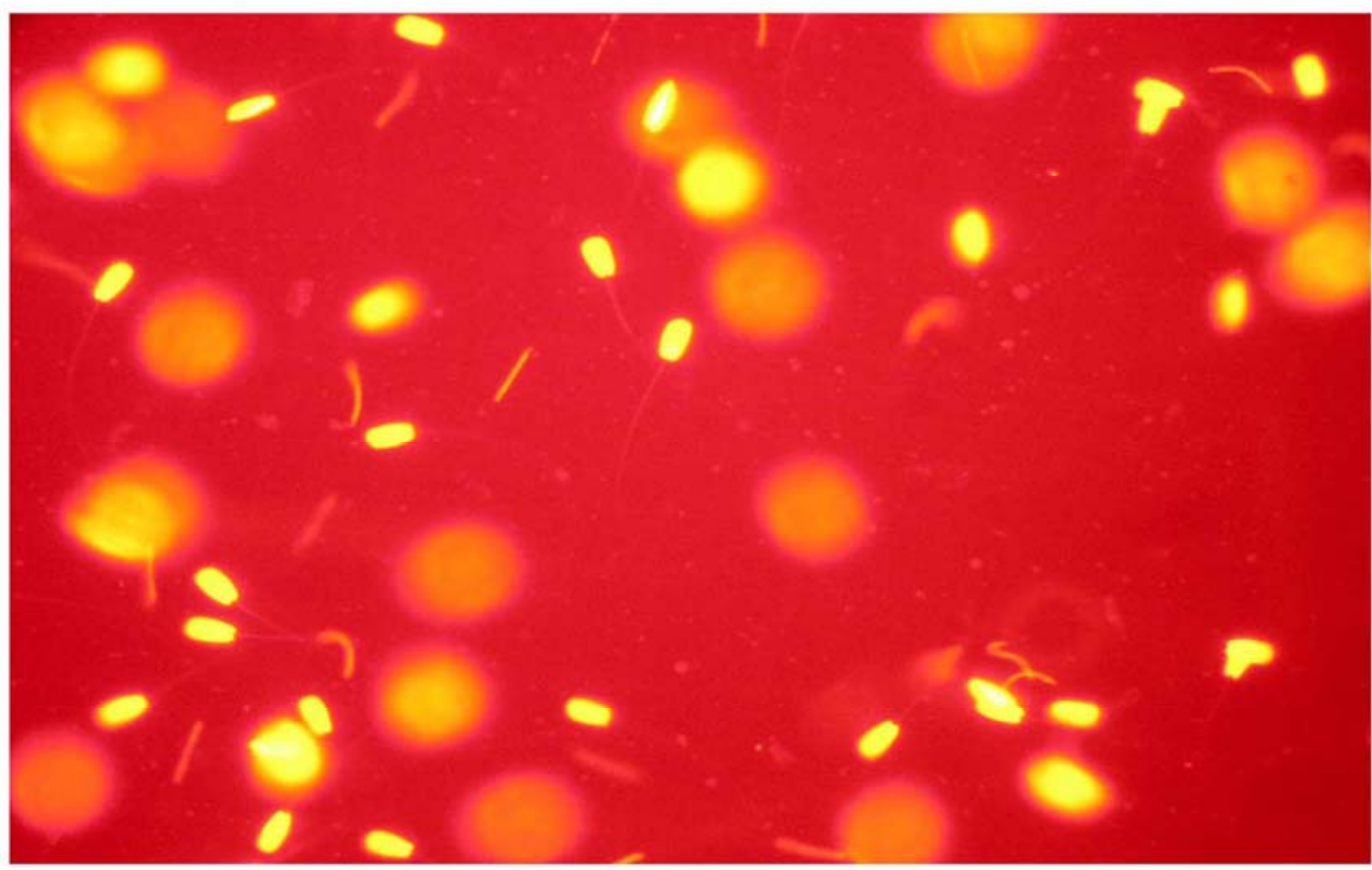

Fig 13.Mitochondrial membrane potential by JC-1 Staining 


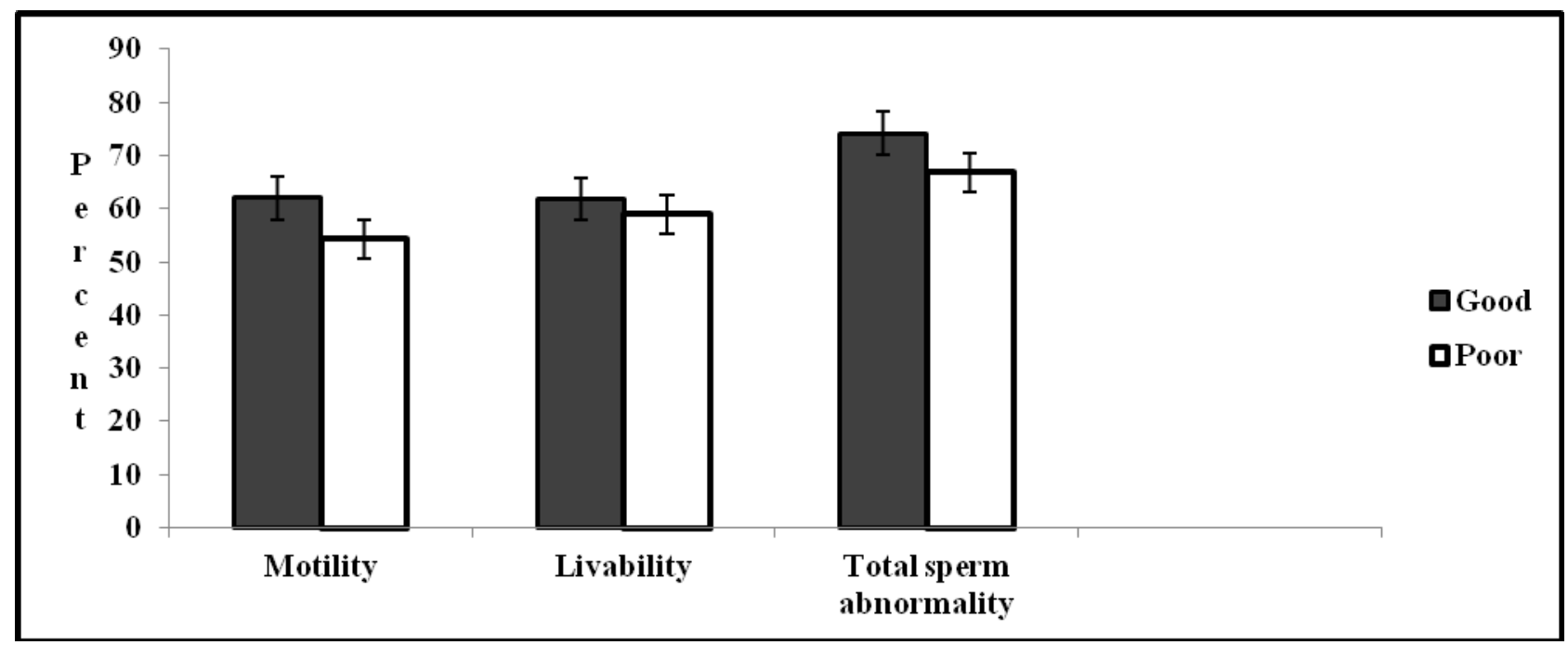

Fig 14a. Post thaw sperm motility, livability and total abnormality of good and poor Jersey crossbred bulls

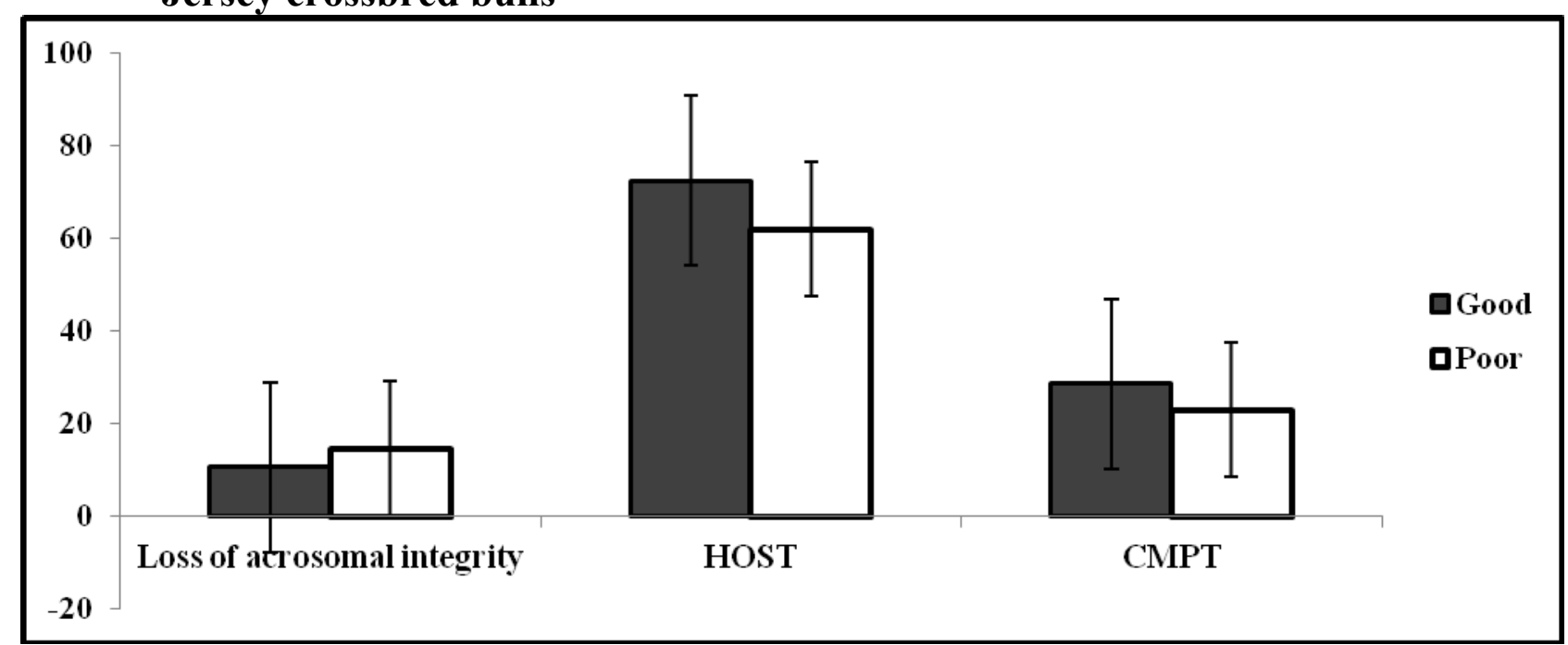

Fig 14b. Post thaw sperm loss of acrosomal integrity, HOST and CMPT of good and poor Jersey crossbred bulls

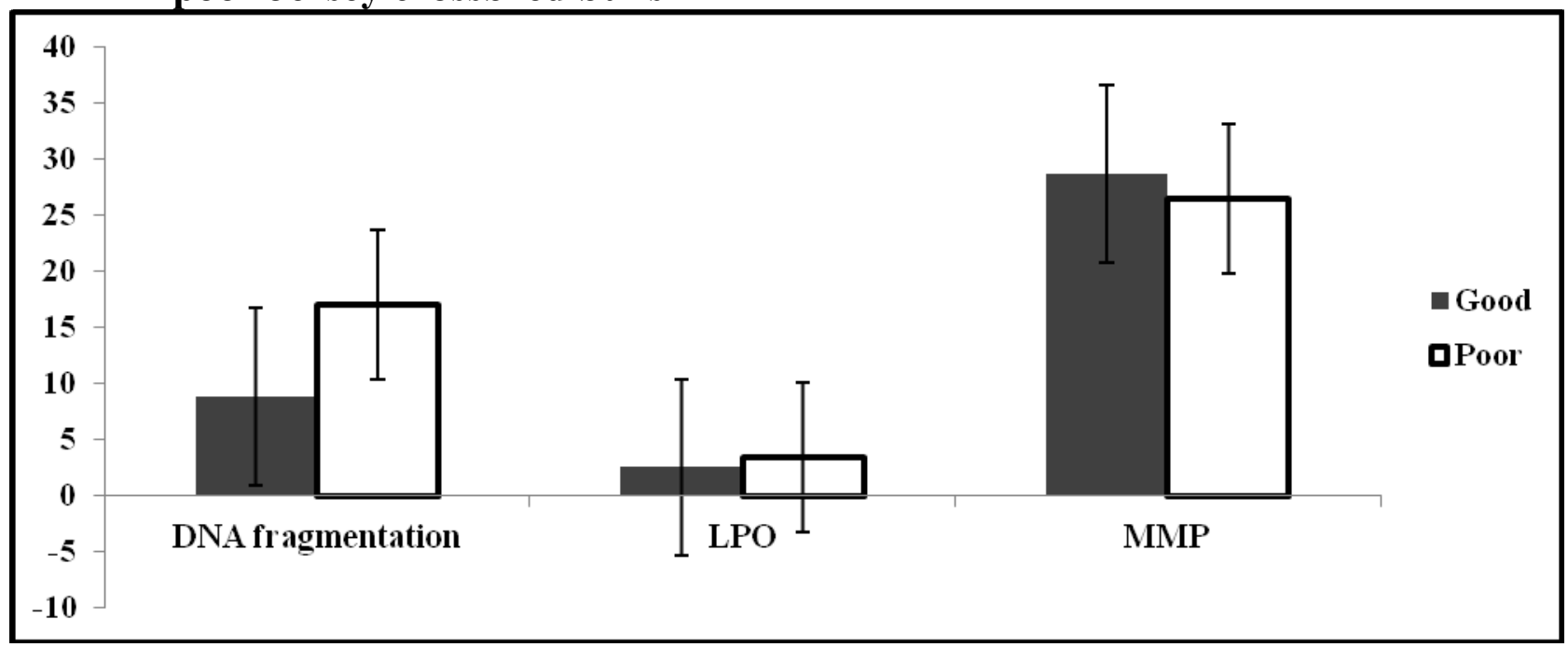

Fig 14c. Post thaw sperm DNA fragmentation, LPO and MMP of good and poor Jersey crossbred bulls 
TABLE 5. ASSESSMENT OF MOTILITY AT DIFFERENT HOURS OF INCUBATION IN GOOD AND POOR FREEZABLE CROSSBRED BULL SEMEN (CASA)

$\mathrm{O} \mathrm{Hr}$

\begin{tabular}{|l|c|c|c|}
\hline \multicolumn{1}{|c|}{ Bull } & Good Freezer (18) & Poor Freezer (18) & 't' value \\
\hline Forameters & & & \\
\hline Non Progressive motility (\%) & $17.74 \pm 1.89$ & $21.70 \pm 21.50$ & $1.78^{\mathrm{NS}}$ \\
\hline Total Motility (\%) & $84.80 \pm 1.87$ & $78.41 \pm 2.75$ & $2.74^{\mathrm{NS}}$ \\
\hline Static sperms (\%) & $15.20 \pm 1.87$ & $21.59 \pm 2.751$ & $2.74^{\mathrm{NS}}$ \\
\hline
\end{tabular}

$1 \mathrm{Hr}$

\begin{tabular}{|l|c|c|c|}
\hline \multicolumn{1}{|c|}{ Bull } & Good Freezer (18) & Poor Freezer (18) & ' $t$ ' value \\
\hline Forward Progressive motility (\%) & & & \\
\hline Non Progressive motility (\%) & $13.97 \pm 2.47$ & $25.25 \pm 3.11$ & $8.14^{* *}$ \\
\hline Total Motility (\%) & $83.00 \pm 2.18$ & $74.67 \pm 5.39$ & $2.00^{\mathrm{NS}}$ \\
\hline Static sperms (\%) & $17.00 \pm 2.18$ & $25.33 \pm 5.39$ & $2.00^{\mathrm{NS}}$ \\
\hline
\end{tabular}

\begin{tabular}{|l|c|c|c|}
\hline \multicolumn{1}{|c|}{ Bull } & Good Freezer (18) & Poor Freezer (18) & ' $t$ ' value \\
\hline Forward Progressive motility (\%) & $54.13 \pm 3.72$ & $43.88 \pm 2.62$ & $5.05^{*}$ \\
\hline Non Progressive motility (\%) & $15.33 \pm 2.64$ & $21.87 \pm 2.97$ & $2.66^{\mathrm{NS}}$ \\
\hline Total Motility (\%) & $69.45 \pm 3.12$ & $65.74 \pm 3.62$ & $0.50^{\mathrm{NS}}$ \\
\hline Static sperms (\%) & $30.55 \pm 3.12$ & $34.26 \pm 3.62$ & $0.50^{\mathrm{NS}}$ \\
\hline
\end{tabular}

4 Hrs

\begin{tabular}{|l|c|c|c|}
\hline \multicolumn{1}{|c|}{ Bull } & Good Freezer (18) & Poor Freezer (18) & 't' value \\
\hline Forward Progressive motility (\%) & $42.84 \pm 4.30$ & $28.55 \pm 3.63$ & $6.52^{*}$ \\
\hline Non Progressive motility (\%) & $18.09 \pm 2.69$ & $21.51 \pm 2.37$ & $1.03^{\mathrm{NS}}$ \\
\hline Total Motility (\%) & $60.93 \pm 3.53$ & $50.05 \pm 3.80$ & $4.36^{*}$ \\
\hline Static sperms (\%) & $39.07 \pm 3.53$ & $49.95 \pm 3.80$ & $4.36^{* *}$ \\
\hline
\end{tabular}

$* * \mathrm{p}<0.01$
$* \mathrm{p}<0.05$

$* \mathrm{p}<0.05$

NS Not Significant

Figures in parenthesis indicate number of ejaculates 
TABLE 6. ASSESSMENT OF MOTILITY AT DIFFERENT HOURS OF INCUBATION IN GOOD AND POOR FREEZABLE CROSSBRED BULL SEMEN (CASA) IN DIFFERENT EXPERIMENTAL GROUPS

$\mathbf{0 ~ H r}$

\begin{tabular}{|c|c|c|c|c|c|c|c|c|c|}
\hline \multirow{3}{*}{ Parameters } & \multicolumn{2}{|c|}{ Group: I } & \multirow{3}{*}{$\begin{array}{c}\text { 'tt' } \\
\text { value }\end{array}$} & \multicolumn{2}{|c|}{ Group: II } & \multirow{3}{*}{$\begin{array}{c}\text { 't' } \\
\text { value }\end{array}$} & \multicolumn{2}{|c|}{ Group: III } & \multirow{3}{*}{$\begin{array}{c}\text { 't' } \\
\text { value }\end{array}$} \\
\hline & Good & Poor & & Good & Poor & & Good & Poor & \\
\hline & & & & & & & & & \\
\hline $\begin{array}{l}\text { Progressive } \\
\text { Forward motility }\end{array}$ & $\begin{array}{l}69.17 \\
\pm 4.96\end{array}$ & $\begin{array}{l}51.03 \\
\pm 7.09\end{array}$ & $1.83^{\mathrm{NS}}$ & $\begin{array}{l}69.76 \\
\pm 3.23\end{array}$ & $\begin{array}{l}55.04 \\
\pm 4.76\end{array}$ & $2.23 *$ & $\begin{array}{l}71.47 \\
\pm 8.40\end{array}$ & $\begin{array}{l}53.10 \\
\pm 3.99\end{array}$ & $1.39^{\mathrm{NS}}$ \\
\hline $\begin{array}{l}\text { Non-Progressive } \\
\text { Motility } \\
\end{array}$ & $\begin{array}{l}13.97 \\
\pm 2.08\end{array}$ & $\begin{array}{l}22.95 \\
\pm 7.36\end{array}$ & - & $\begin{array}{l}16.83 \\
\pm 2.08\end{array}$ & $\begin{array}{l}21.63 \\
\pm 5.59\end{array}$ & - & $\begin{array}{l}13.89 \\
\pm 7.11\end{array}$ & $\begin{array}{l}31.10 \\
\pm 4.98\end{array}$ & - \\
\hline Total Motility & $\begin{array}{l}83.14 \\
\pm 5.90\end{array}$ & $\begin{array}{l}73.98 \\
\pm 9.09\end{array}$ & $0.74^{\mathrm{NS}}$ & $\begin{array}{l}86.59 \\
\pm 2.82\end{array}$ & $\begin{array}{l}76.66 \\
\pm 2.35\end{array}$ & $2.14^{*}$ & $\begin{array}{l}85.36 \\
\pm 1.62\end{array}$ & $\begin{array}{l}84.20 \\
\pm 3.50\end{array}$ & $0.30^{\mathrm{NS}}$ \\
\hline Static Sperms & $\begin{array}{l}16.86 \\
\pm 5.90\end{array}$ & $\begin{array}{l}26.02 \\
\pm 9.09\end{array}$ & - & $\begin{array}{l}13.41 \\
\pm 2.82\end{array}$ & $\begin{array}{l}23.34 \\
\pm 2.35\end{array}$ & - & $\begin{array}{l}14.64 \\
\pm 1.62\end{array}$ & $\begin{array}{l}15.80 \\
\pm 3.50\end{array}$ & - \\
\hline
\end{tabular}


$1 \mathrm{Hr}$

\begin{tabular}{|c|c|c|c|c|c|c|c|c|c|}
\hline \multirow{2}{*}{ Parameters } & \multicolumn{2}{|c|}{ Group: I } & \multirow{2}{*}{$\begin{array}{c}\text { 't' } \\
\text { value }\end{array}$} & \multicolumn{2}{|c|}{ Group: II } & \multirow{2}{*}{$\begin{array}{c}\text { 't' } \\
\text { value }\end{array}$} & \multicolumn{2}{|c|}{ Group: III } & \multirow{2}{*}{$\begin{array}{c}\quad \mathbf{t}^{\prime} ' \\
\text { value }\end{array}$} \\
\hline & Good & Poor & & Good & Poor & & Good & Poor & \\
\hline $\begin{array}{l}\text { Progressive } \\
\text { Forward motility }\end{array}$ & $\begin{array}{l}65.38 \\
\pm 4.04\end{array}$ & $\begin{array}{l}57.08 \\
\pm 5.32\end{array}$ & $1.23^{\mathrm{NS}}$ & $\begin{array}{r}66.43 \\
\pm 7.21\end{array}$ & $\begin{array}{r}44.14 \\
\pm 7.75\end{array}$ & $2.10^{*}$ & $\begin{array}{l}66.05 \\
\pm 1.05\end{array}$ & $\begin{array}{l}58.02 \\
\pm 4.13\end{array}$ & $1.88^{\mathrm{NS}}$ \\
\hline $\begin{array}{l}\text { Non-Progressive } \\
\text { Motility }\end{array}$ & $\begin{array}{l}18.31 \\
\pm 5.05\end{array}$ & $\begin{array}{l}21.42 \\
\pm 1.90\end{array}$ & - & $\begin{array}{l}14.05 \\
\pm 4.32\end{array}$ & $\begin{array}{l}21.70 \\
\pm 5.99\end{array}$ & - & $\begin{array}{l}18.07 \\
\pm 3.54\end{array}$ & $\begin{array}{l}22.05 \\
\pm 4.52\end{array}$ & - \\
\hline Total Motility & $\begin{array}{r}83.69 \\
\pm 3.33 \\
\end{array}$ & $\begin{array}{l}78.50 \\
\pm 4.34\end{array}$ & $0.94^{\mathrm{NS}}$ & $\begin{array}{r}80.49 \\
\pm 3.70\end{array}$ & $\begin{array}{r}65.83 \\
\pm 1.30\end{array}$ & $3.74^{*}$ & $\begin{array}{l}84.13 \\
\pm 4.53\end{array}$ & $\begin{array}{l}80.06 \\
\pm 7.97\end{array}$ & $0.12^{\mathrm{NS}}$ \\
\hline Static Sperms & $\begin{array}{r}16.31 \\
\pm 3.33\end{array}$ & $\begin{array}{l}21.50 \\
\pm 4.34\end{array}$ & - & $\begin{array}{r}19.51 \\
\pm 3.70\end{array}$ & $\begin{array}{l}34.17 \\
\pm 1.30\end{array}$ & - & $\begin{array}{r}15.87 \\
\pm 4.53\end{array}$ & $\begin{array}{r}19.94 \\
\pm 7.97\end{array}$ & - \\
\hline
\end{tabular}


2 Hrs

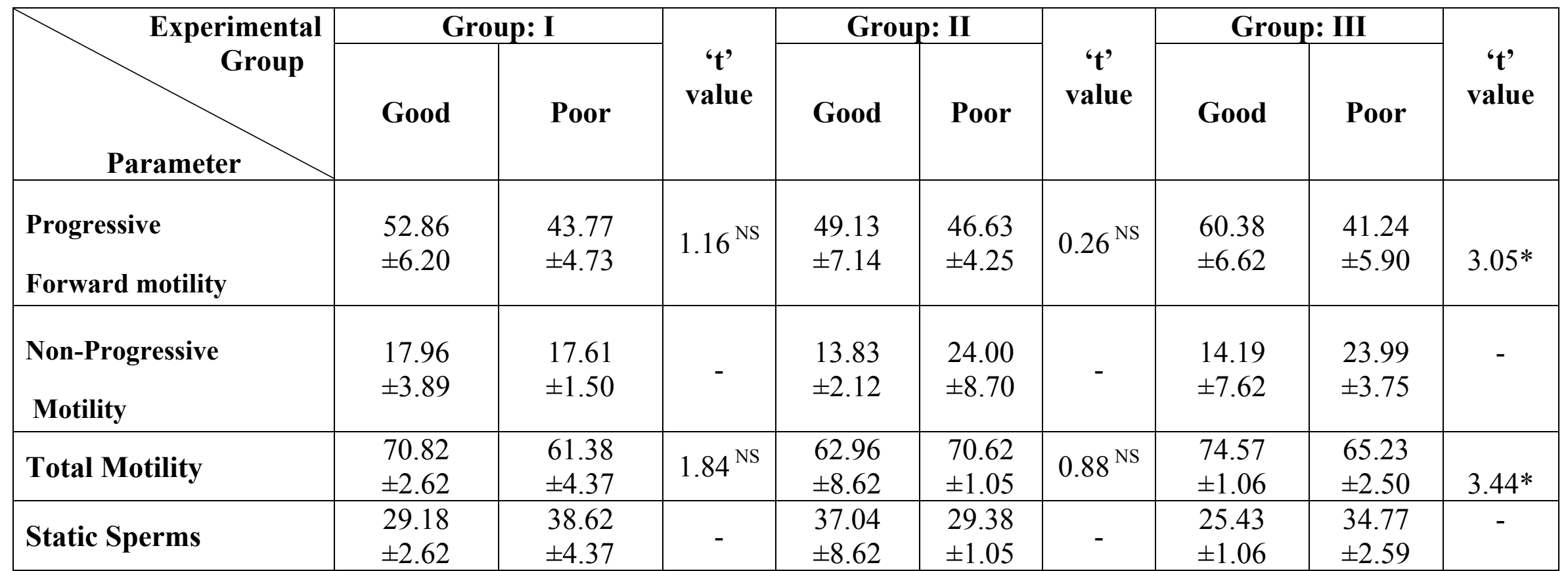


4 Hrs

\begin{tabular}{|c|c|c|c|c|c|c|c|c|c|}
\hline Experimental & & & ' $t$ ', & Gro & II & ' $t$ ' & $\mathbf{G r}$ & III & ' $t$ ' \\
\hline Parameter & Good & Poor & value & Good & Poor & value & Good & Poor & value \\
\hline $\begin{array}{l}\text { Progressive } \\
\text { Forward motility }\end{array}$ & $\begin{array}{l}39.58 \\
\pm 1.06\end{array}$ & $\begin{array}{l}25.09 \\
\pm 3.99\end{array}$ & $3.51 *$ & $\begin{array}{l}42.96 \\
\pm 5.60\end{array}$ & $\begin{array}{l}29.77 \\
\pm 3.69\end{array}$ & $1.96^{\mathrm{NS}}$ & $\begin{array}{l}46.98 \\
\pm 8.87\end{array}$ & $\begin{array}{l}30.79 \\
\pm 1.09\end{array}$ & $1.81^{\mathrm{NS}}$ \\
\hline $\begin{array}{l}\text { Non-Progressive } \\
\text { Motility }\end{array}$ & $\begin{array}{l}21.52 \\
\pm 4.35\end{array}$ & $\begin{array}{l}22.70 \\
\pm 4.87\end{array}$ & - & $\begin{array}{l}14.71 \\
\pm 6.52\end{array}$ & $\begin{array}{l}21.35 \\
\pm 3.96\end{array}$ & - & $\begin{array}{l}18.04 \\
\pm 3.92\end{array}$ & $\begin{array}{l}20.48 \\
\pm 5.20\end{array}$ & - \\
\hline Total Motility & $\begin{array}{l}61.10 \\
\pm 6.25\end{array}$ & $\begin{array}{l}47.49 \\
\pm 1.03\end{array}$ & $2.15^{*}$ & $\begin{array}{l}57.67 \\
\pm 6.93\end{array}$ & $\begin{array}{l}51.11 \\
\pm 5.65\end{array}$ & $0.66^{\mathrm{NS}}$ & $\begin{array}{l}64.02 \\
\pm 7.25\end{array}$ & $\begin{array}{l}49.56 \\
\pm 4.31\end{array}$ & $1.71^{\mathrm{NS}}$ \\
\hline Static Sperms & $\begin{array}{l}42.33 \\
\pm 6.93\end{array}$ & $\begin{array}{l}46.51 \\
\pm 1.03\end{array}$ & - & $\begin{array}{l}38.90 \\
\pm 6.25\end{array}$ & $\begin{array}{l}48.89 \\
\pm 5.65\end{array}$ & - & $\begin{array}{l}35.98 \\
\pm 7.25\end{array}$ & $\begin{array}{l}54.44 \\
\pm 4.31\end{array}$ & - \\
\hline
\end{tabular}

$* \mathrm{p}<0.05$

NS Not Significant 

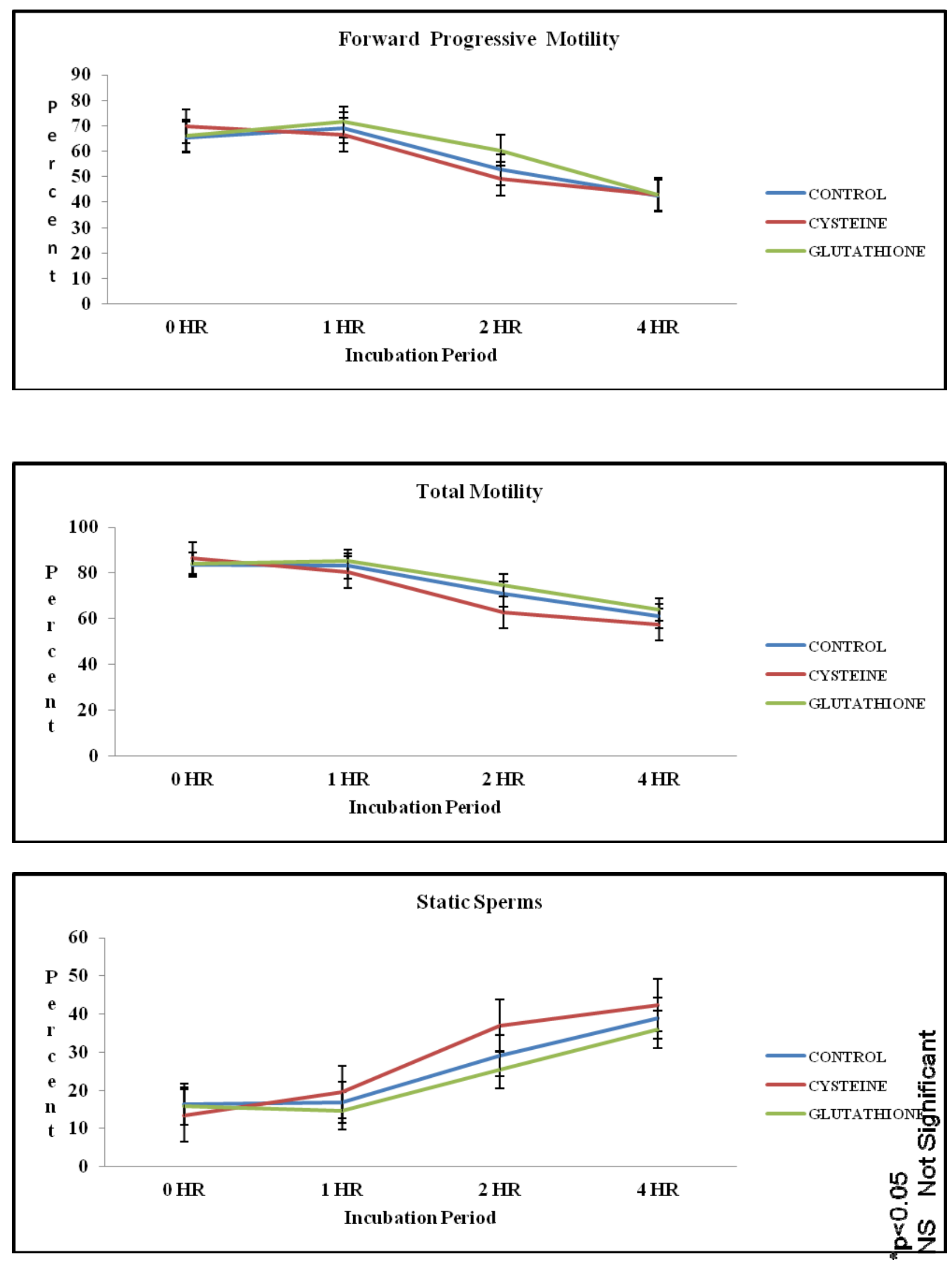

Fig 15. CASA motility parameters of control, cysteine and glutathione of good freezable Jersey crossbred bulls 

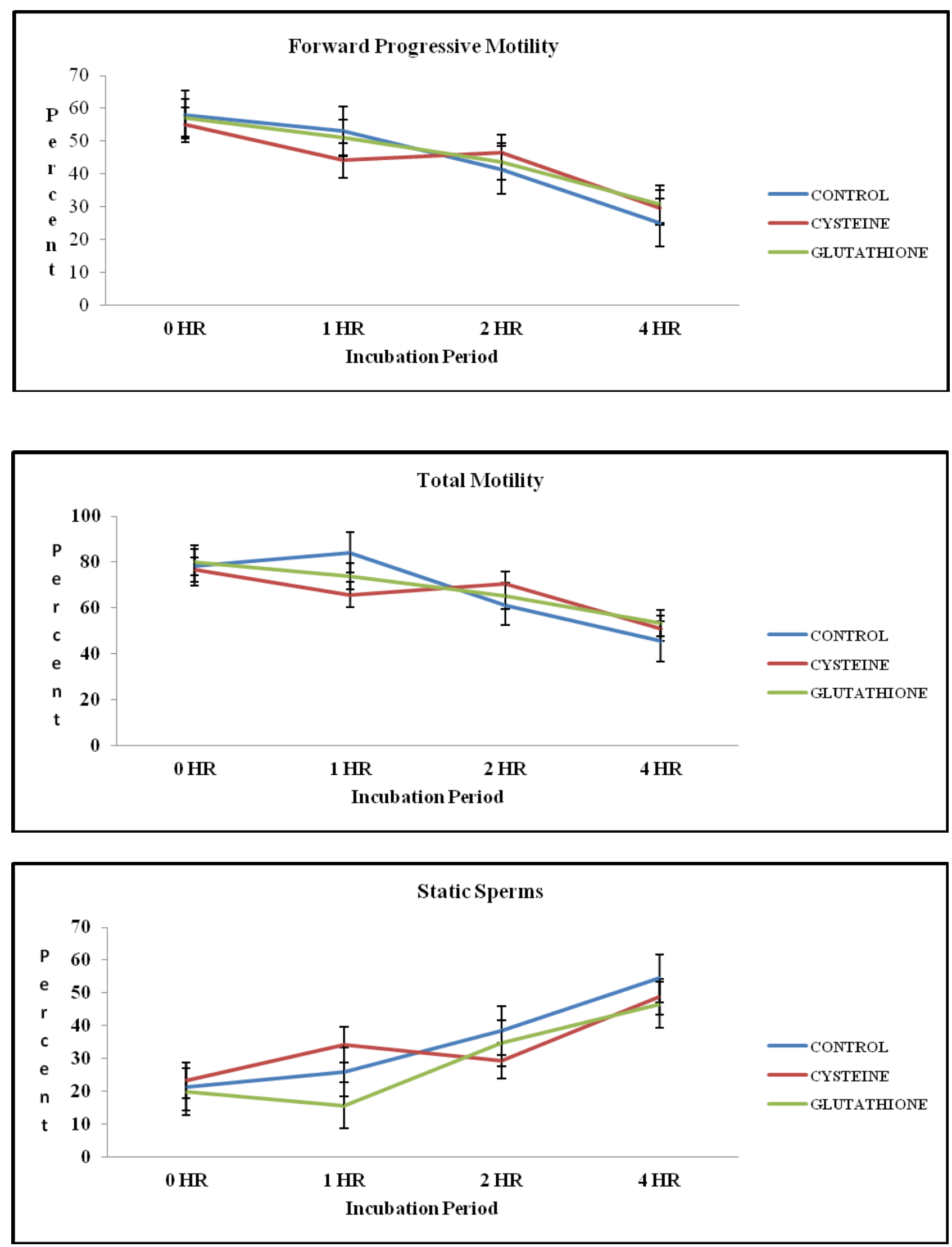

Fig 16. CASA motility parameters of control, cysteine and glutathione of poor freezable Jersey crossbred bulls 


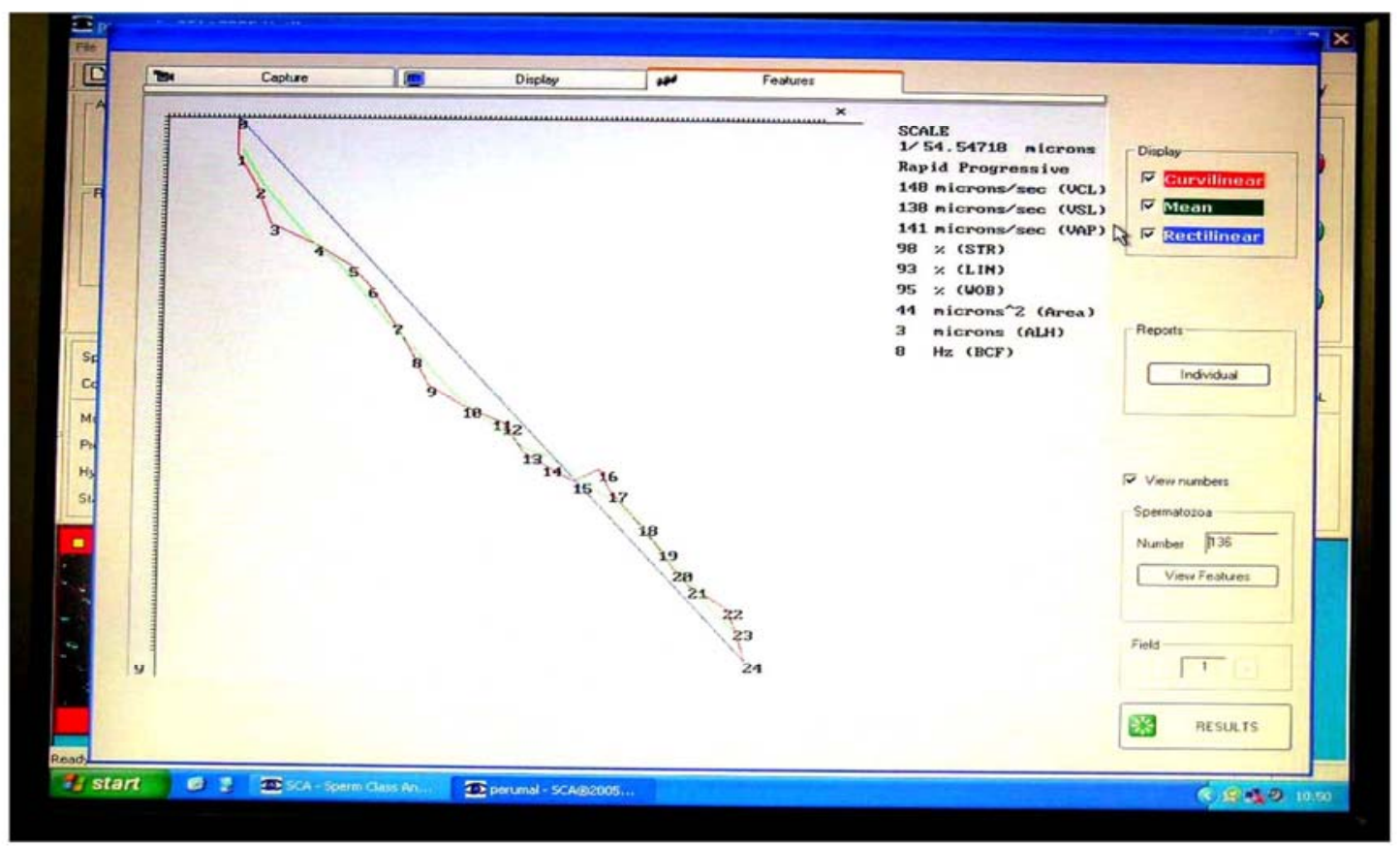

Fig 17.Computer Assisted Sperm Analysis of post thaw semen

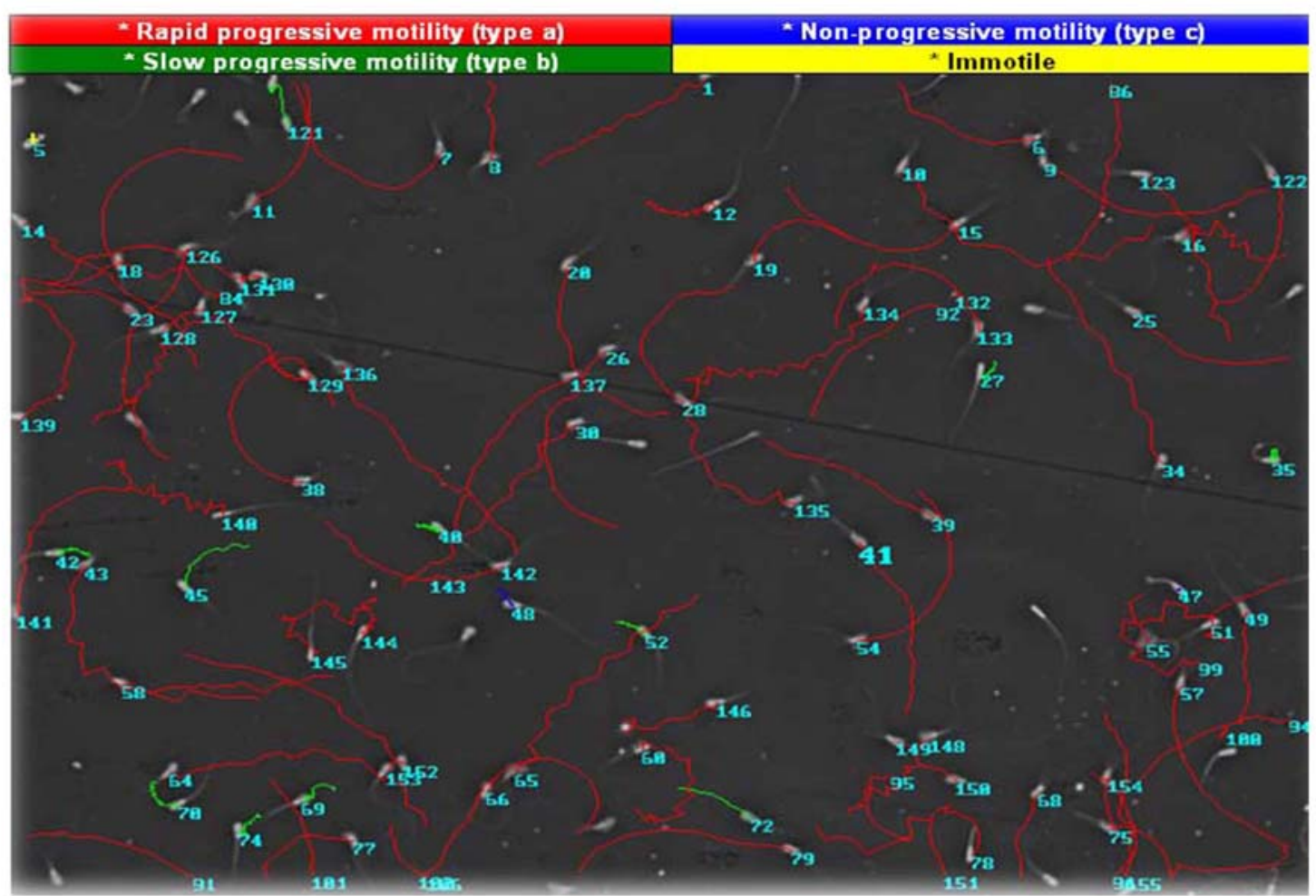

Fig 18. Post thaw motility parameters by Computer Assissted Sperm Analyser (CASA) 
TABLE 7. COMPUTER ASSISTED SEMEN ANALYSIS (CASA) OF SOME POST-THAW SEMINAL CHARACTERISTICS IN CONTROL AND TREATMENT GROUPS AT DIFFERENT HOURS OF INCUBATION

( Hr

\begin{tabular}{|c|c|c|c|c|c|c|}
\hline \multirow{2}{*}{ Parameter } & \multicolumn{2}{|c|}{ Group: I } & \multicolumn{2}{|c|}{ Group: II } & \multicolumn{2}{|c|}{ Group: III } \\
\hline & Good & Poor & Good & Poor & Good & Poor \\
\hline Curvilinear Velocity (VCL) ( $\mu \mathrm{m} / \mathrm{sec})$. & $125.03 \pm 4.33$ & $111.36 \pm 12.78$ & $112.75 \pm 4.42$ & $110.08 \pm 10.30$ & $130.76 \pm 3.47$ & $138.63 \pm 4.1$ \\
\hline Straight line Velocity (VSL) $(\mu \mathrm{m} / \mathrm{sec})$. & $93.43 \pm 3.21$ & $79.72 \pm 11.60$ & $76.20 \pm 10.37$ & $73.04 \pm 8.29$ & $91.84 \pm 3.39$ & $87.23 \pm 11.03$ \\
\hline Average path Velocity (VAP) $(\mu \mathrm{m}$ & $111.66 \pm 5.12$ & $95.65 \pm 11.46$ & $89.93 \pm 10.12$ & $86.28 \pm 7.14$ & $111.47 \pm 5.4$ & $103.42 \pm 12.5$ \\
\hline Linearity (LIN) (\%) & $74.83 \pm 2.58$ & $71.11 \pm 2.11$ & $67.72 \pm 9.10$ & $66.20 \pm 2.38$ & $66.21 \pm 7.01$ & $66.22 \pm 0.58$ \\
\hline Straightness (STR) (\%) & $84.09 \pm 3.14$ & $82.87 \pm 2.06$ & $84.28 \pm 2.15$ & $84.24 \pm 2.48$ & $83.75 \pm 1.67$ & $82.50 \pm 1.72$ \\
\hline Wobble (WOB) (\%) & $89.05 \pm 1.16$ & $85.79 \pm 0.99$ & $79.96 \pm 8.92$ & $78.64 \pm 2.81$ & $80.32 \pm 1.70$ & $78.64 \pm 7.86$ \\
\hline $\begin{array}{l}\text { Amplitude of Lateral Head } \\
\text { displacement }(\mathrm{ALH})(\mu \mathrm{m})\end{array}$ & $2.88 \pm 0.03$ & $2.84 \pm 0.27$ & $4.01 \pm 0.17$ & $3.11 \pm 0.40$ & $4.00 \pm 0.16$ & $3.59 \pm 0.24$ \\
\hline Beat/Cross Frequency (BCF) $\mathrm{Hz}$ & $9.77 \pm 0.49$ & $9.54 \pm 0.82$ & $10.48 \pm 1.37$ & $11.92 \pm 0.30$ & $11.52 \pm 1.22$ & $11.43 \pm 0.65$ \\
\hline
\end{tabular}

\begin{tabular}{|c|c|c|c|c|c|c|}
\hline \multicolumn{7}{|c|}{$1 \mathrm{Hr}$} \\
\hline \multirow{2}{*}{ Parameter } & \multicolumn{2}{|c|}{ Group: I } & \multicolumn{2}{|c|}{ Group: II } & \multicolumn{2}{|c|}{ Group: III } \\
\hline & Good & Poor & Good & Poor & Good & Poor \\
\hline Curvilinear Velocity (VCL) $(\mu \mathrm{m} / \mathrm{sec})$. & $105.82 \pm 12.47$ & $93.81 \pm 7.27$ & $112.59 \pm 12.08$ & $94.25 \pm 9.89$ & $102.39 \pm 5.26$ & $96.02 \pm 20.4$ \\
\hline Straight line Velocity (VSL) ( $\mu \mathrm{m} / \mathrm{sec})$. & $59.14 \pm 5.88$ & $56.73 \pm 7.16$ & $66.16 \pm 4.49$ & $60.22 \pm 11.62$ & $64.20 \pm 10.94$ & $57.09 \pm 13.9$ \\
\hline Average path Velocity (VAP) $(\mu \mathrm{m}$ & $75.24 \pm 8.07$ & $69.15 \pm 6.08$ & $83.37 \pm 7.55$ & $68.42 \pm 10.38$ & $74.10 \pm 8.82$ & $70.59 \pm 15.2$ \\
\hline Linearity (LIN) (\%) & $62.87 \pm 3.24$ & $53.74 \pm 4.49$ & $63.01 \pm 7.39$ & $59.63 \pm 4.83$ & $62.87 \pm 10.18$ & $58.60 \pm 3.17$ \\
\hline Straightness (STR) (\%) & $85.11 \pm 1.70$ & $75.11 \pm 3.31$ & $86.61 \pm 3.52$ & $79.84 \pm 3.58$ & $85.32 \pm 5.26$ & $79.79 \pm 3.87$ \\
\hline Wobble (WOB) (\%) & $73.73 \pm 2.20$ & $71.31 \pm 2.83$ & $72.19 \pm 5.86$ & $74.46 \pm 3.13$ & $72.53 \pm 8.06$ & $73.40 \pm 0.44$ \\
\hline $\begin{array}{c}\text { Amplitude of Lateral Head } \\
\text { displacement (ALH) }(\mu \mathrm{m})\end{array}$ & $3.36 \pm 0.39$ & $2.76 \pm 0.28$ & $3.47 \pm 0.33$ & $2.75 \pm 0.16$ & $2.87 \pm 0.22$ & $2.83 \pm 0.44$ \\
\hline Beat/Cross Frequency (BCF) Hz & $14.06 \pm 0.15$ & $13.19 \pm 0.61$ & $14.25 \pm 0.78$ & $14.28 \pm 1.05$ & $14.56 \pm 0.131$ & $14.54 \pm 0.31$ \\
\hline
\end{tabular}


2 Hrs

\begin{tabular}{|c|c|c|c|c|c|c|}
\hline \multirow{2}{*}{ Parameter } & \multicolumn{2}{|c|}{ Group: I } & \multicolumn{2}{|c|}{ Group: II } & \multicolumn{2}{|c|}{ Group: III } \\
\hline & Good & Poor & Good & Poor & Good & Poor \\
\hline Curvilinear Velocity (VCL) $(\mu \mathrm{m} / \mathrm{sec})$. & $103.82 \pm 9.25$ & $92.78 \pm 3.90$ & $100.59 \pm 4.42$ & $96.93 \pm 18.81$ & $125.10 \pm 3.83$ & $107.21 \pm 12.23$ \\
\hline Straight line Velocity (VSL) $(\mu \mathrm{m} / \mathrm{sec})$. & $64.28 \pm 5.19$ & $56.42 \pm 1.94$ & $61.02 \pm 5.12$ & $54.49 \pm 12.43$ & $77.44 \pm 2.43$ & $70.08 \pm 17.32$ \\
\hline Average path Velocity (VAP) $(\mu \mathrm{m} / \mathrm{sec})$. & $79.12 \pm 6.28$ & $69.30 \pm 3.41$ & $73.83 \pm 4.16$ & $68.02 \pm 14.57$ & $96.51 \pm 0.75$ & $81.43 \pm 15.43$ \\
\hline Linearity (LIN) (\%) & $61.00 \pm 1.06$ & $62.39 \pm 5.34$ & $60.81 \pm 4.05$ & $55.82 \pm 8.13$ & $63.94 \pm 9.44$ & $62.04 \pm 3.07$ \\
\hline Straightness (STR) (\%) & $81.47 \pm 1.46$ & $80.21 \pm 1.89$ & $82.52 \pm 2.99$ & $79.56 \pm 5.58$ & $84.76 \pm 6.22$ & $81.38 \pm 3.94$ \\
\hline Wobble (WOB) (\%) & $77.74 \pm 0.69$ & $76.40 \pm 2.76$ & $73.51 \pm 2.23$ & $69.48 \pm 5.24$ & $77.30 \pm 2.58$ & $74.89 \pm 5.91$ \\
\hline $\begin{array}{l}\text { Amplitude of Lateral Head } \\
\text { displacement }(\mathrm{ALH})(\mu \mathrm{m})\end{array}$ & $2.68 \pm 0.20$ & $2.84 \pm 0.19$ & $2.92 \pm 0.24$ & $3.13 \pm 0.46$ & $2.85 \pm 0.16$ & $3.56 \pm 0.038$ \\
\hline Beat/Cross Frequency (BCF) $\mathrm{Hz}$ & $14.68 \pm 0.74$ & $14.56 \pm 0.10$ & $14.90 \pm 0.21$ & $13.14 \pm 0.99$ & $14.94 \pm 0.81$ & $14.88 \pm 0.11$ \\
\hline
\end{tabular}

4 Hrs

\begin{tabular}{|c|c|c|c|c|c|c|}
\hline \multirow{2}{*}{ Parameter } & \multicolumn{2}{|c|}{ Group: I } & \multicolumn{2}{|c|}{ Group: II } & \multicolumn{2}{|c|}{ Group: III } \\
\hline & Good & Poor & Good & Poor & Good & Poor \\
\hline Curvilinear Velocity (VCL) $(\mu \mathrm{m} / \mathrm{sec})$. & $104.96 \pm 11.96$ & $98.89 \pm 1.20$ & $106.11 \pm 5.89$ & $100.59 \pm 4.42$ & $120.37 \pm 9.23$ & $\begin{array}{c}118.13 \pm 14.4 \\
6\end{array}$ \\
\hline Straight line Velocity (VSL) $(\mu \mathrm{m} / \mathrm{sec})$. & $52.60 \pm 9.04$ & $49.70 \pm 12.04$ & $61.02 \pm 5.12$ & $50.69 \pm 9.80$ & $56.72 \pm 10.45$ & $58.66 \pm 10.54$ \\
\hline Average path Velocity (VAP) ( $\mu \mathrm{m}$ sec.) & $69.29 \pm 5.02$ & $68.20 \pm 13.01$ & $73.83 \pm 4.16$ & $68.10 \pm 11.61$ & $83.18 \pm 13.71$ & $77.59 \pm 9.36$ \\
\hline Linearity (LIN) (\%) & $48.51 \pm 8.47$ & $46.77 \pm 9.33$ & $60.81 \pm 4.05$ & $47.18 \pm 7.02$ & $53.37 \pm 8.64$ & $48.63 \pm 6.80$ \\
\hline Straightness (STR) (\%) & $72.64 \pm 6.55$ & $70.44 \pm 5.29$ & $82.52 \pm 2.99$ & $74.03 \pm 2.52$ & $76.34 \pm 7.02$ & $71.82 \pm 5.50$ \\
\hline Wobble (WOB) (\%) & $69.46 \pm 4.96$ & $64.09 \pm 7.98$ & $73.51 \pm 2.23$ & $63.42 \pm 7.82$ & $65.75 \pm 6.26$ & $68.54 \pm 5.23$ \\
\hline $\begin{array}{l}\text { Amplitude of Lateral Head } \\
\text { displacement }(\text { ALH) }(\mu \mathrm{m})\end{array}$ & $3.55 \pm 0.33$ & $3.09 \pm 0.23$ & $3.92 \pm 0.24$ & $3.92 \pm 0.30$ & $3.84 \pm 0.18$ & $3.30 \pm 0.18$ \\
\hline Beat/Cross Frequency (BCF) $\mathrm{Hz}$ & $13.54 \pm 1.07$ & $11.95 \pm 0.31$ & $14.90 \pm 0.21$ & $11.68 \pm 0.47$ & $13.88 \pm 0.44$ & $13.95 \pm 0.97$ \\
\hline
\end{tabular}



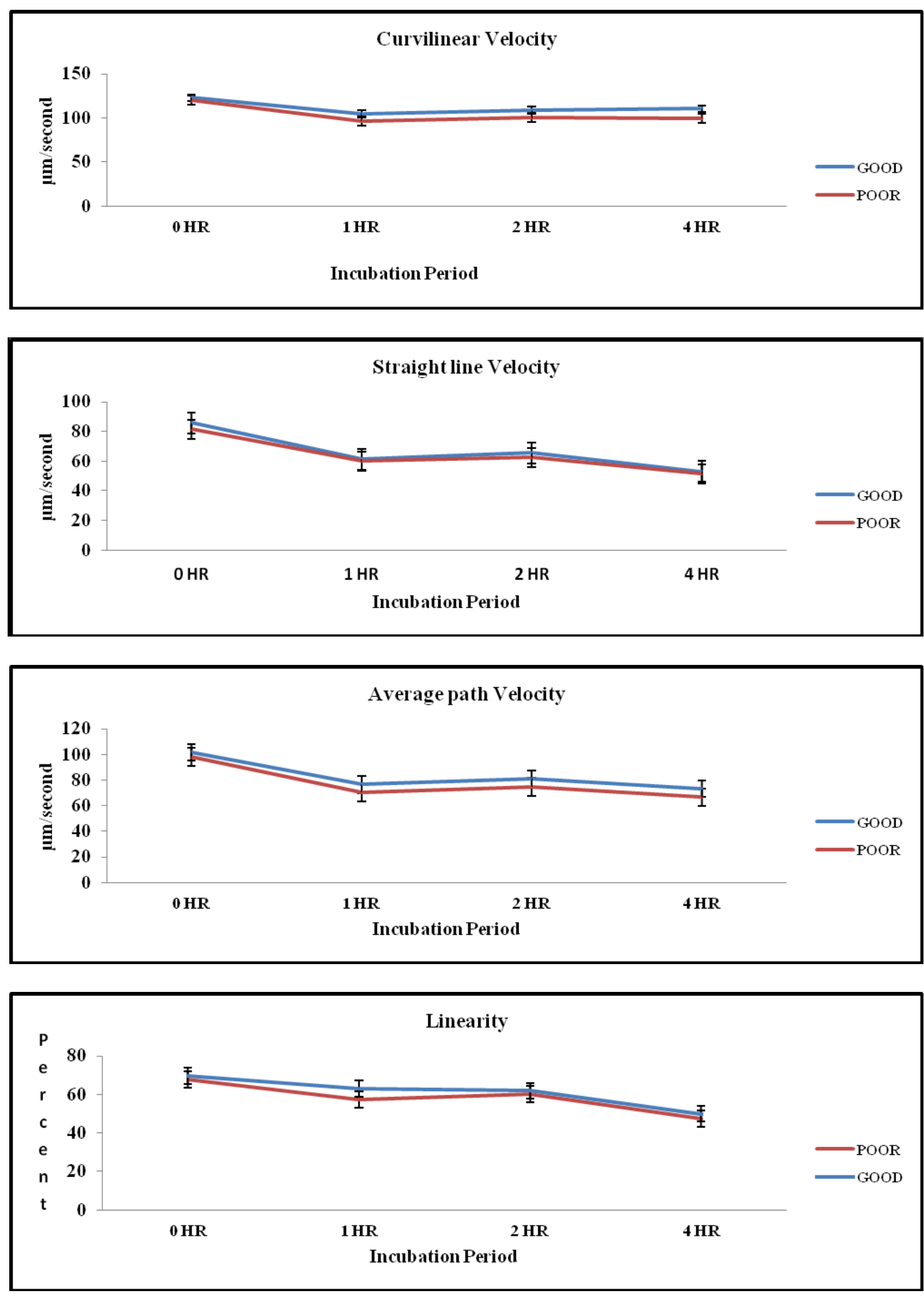

Fig 19a. CASA velocity Parameters of good and poor freezable Jersey crossbred bulls 

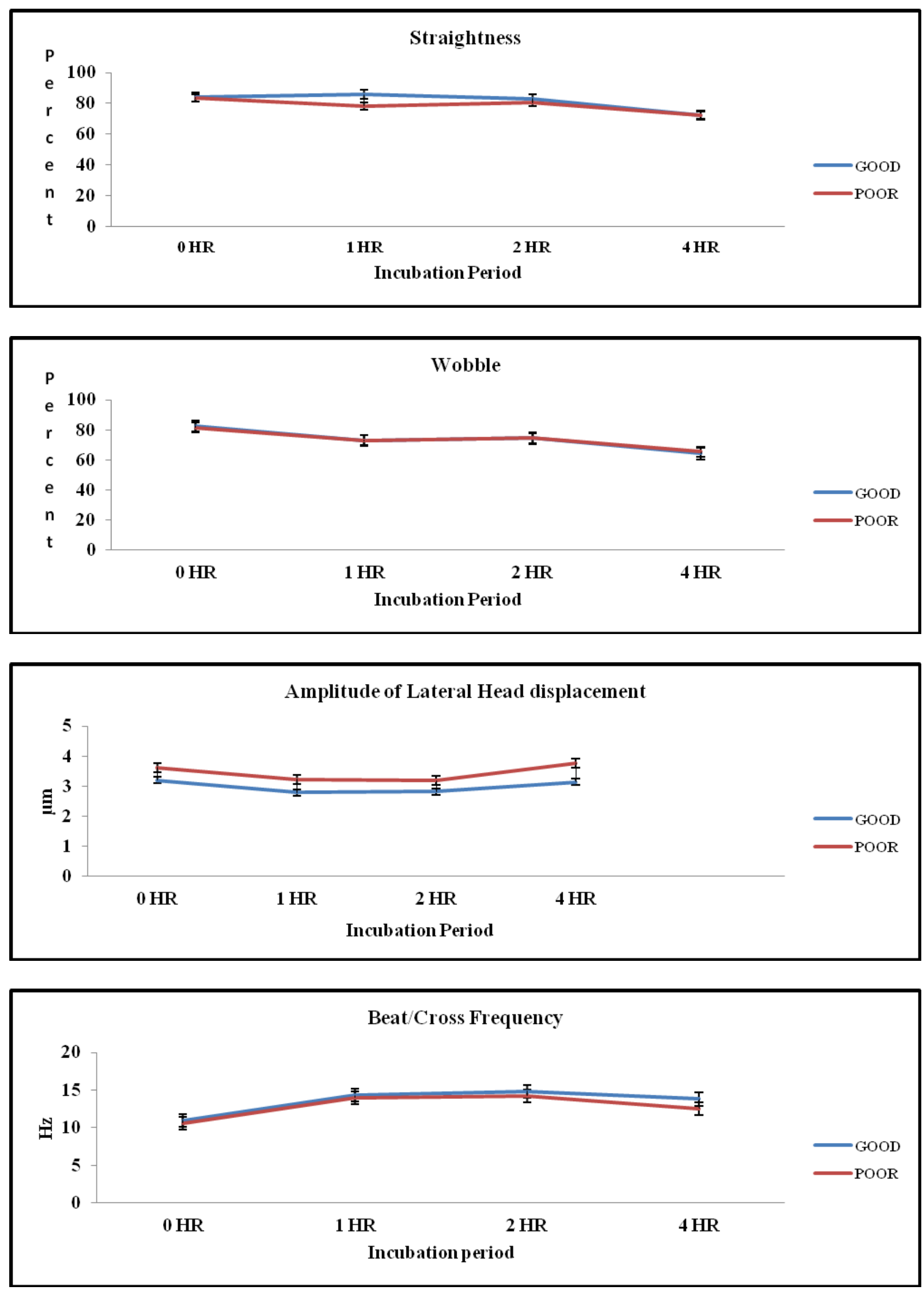

Fig 19b. CASA velocity Parameters of good and poor freezable Jersey crossbred bulls 

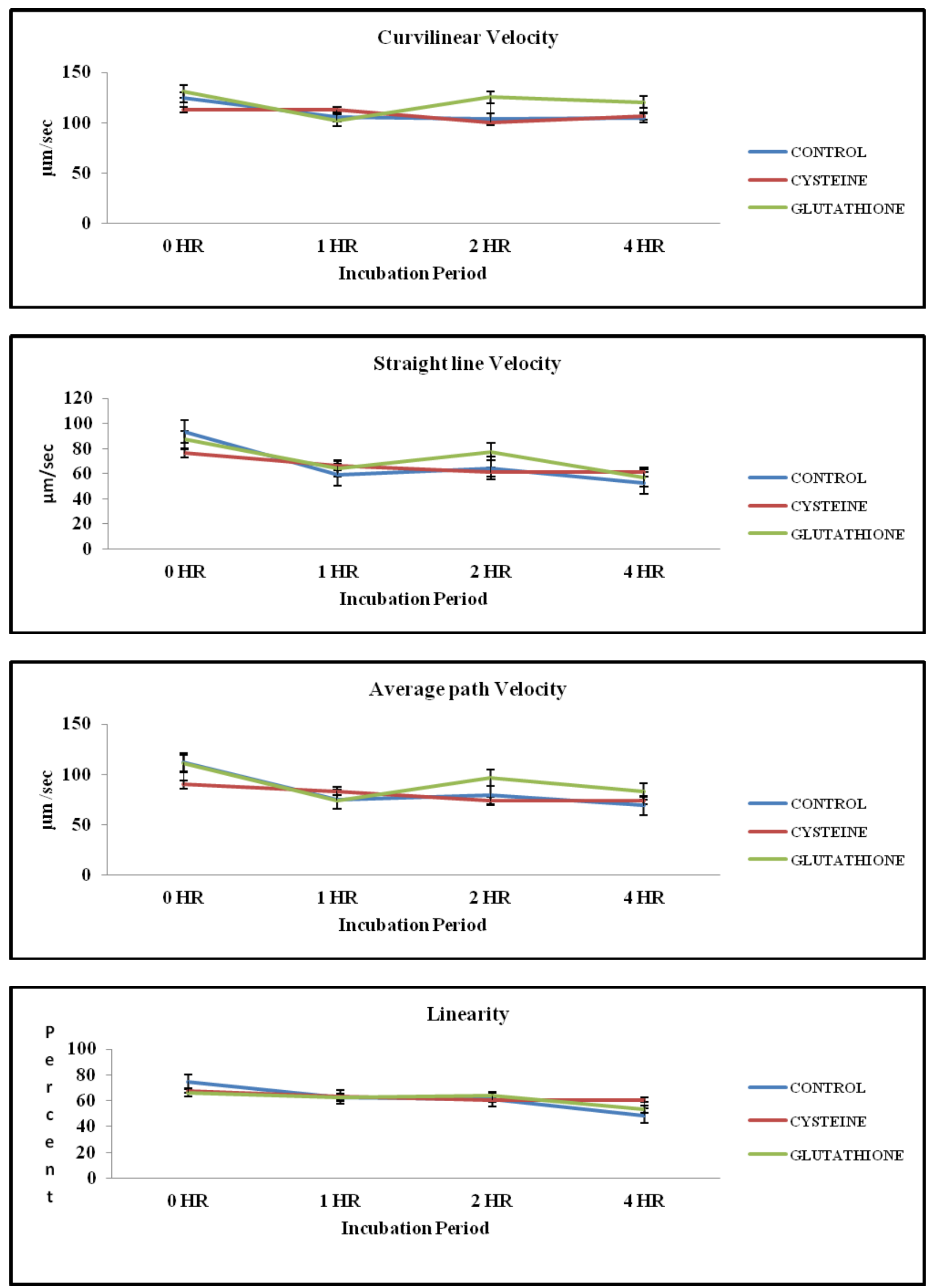

Fig 20a. CASA velocity Parameters of control, cysteine and glutathione of good freezable Jersey crossbred bulls 

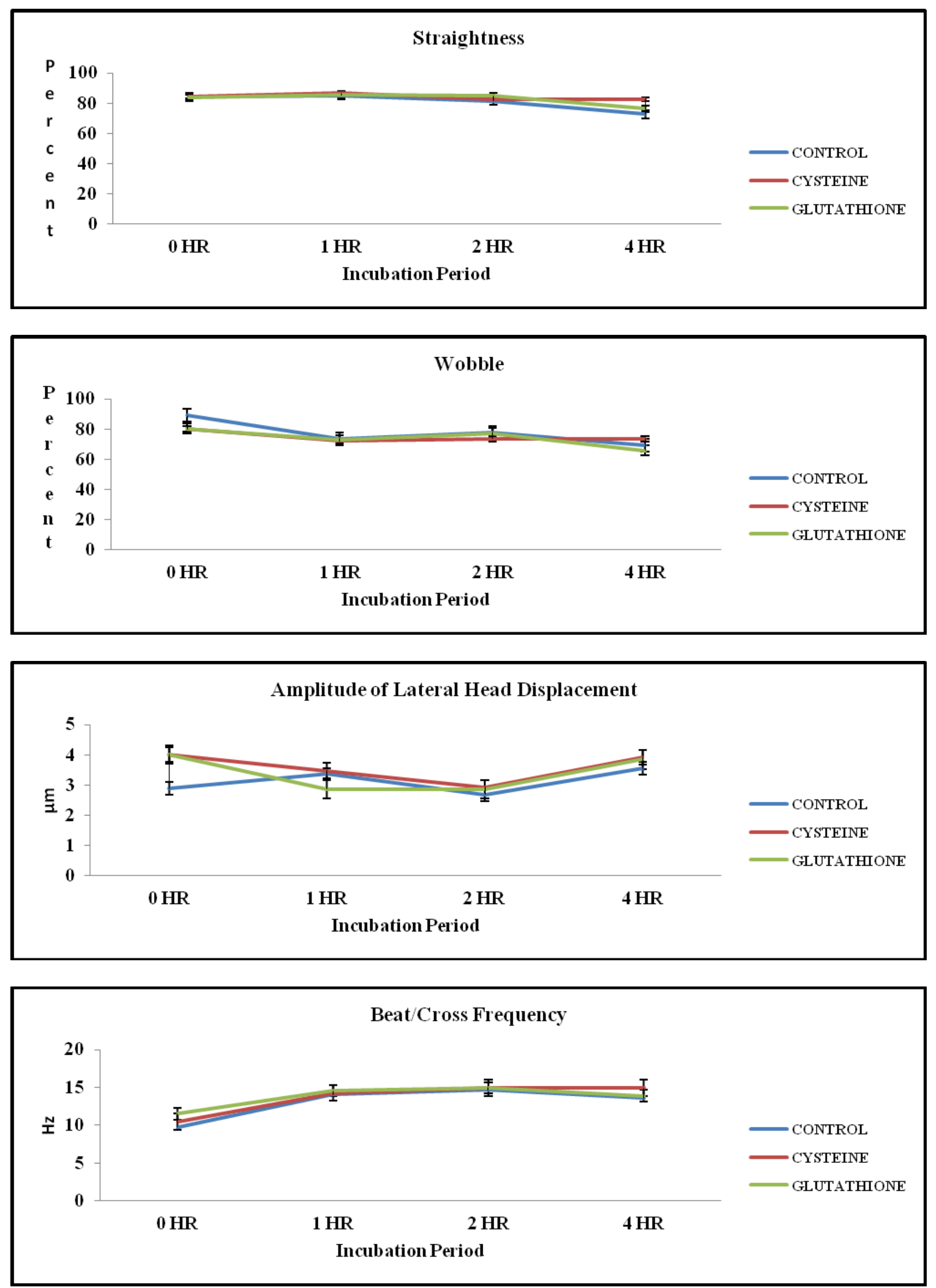

Fig 20b. CASA velocity parameters of control, cysteine hydrochloride and glutathione of good freezable Jersey crossbred bulls 

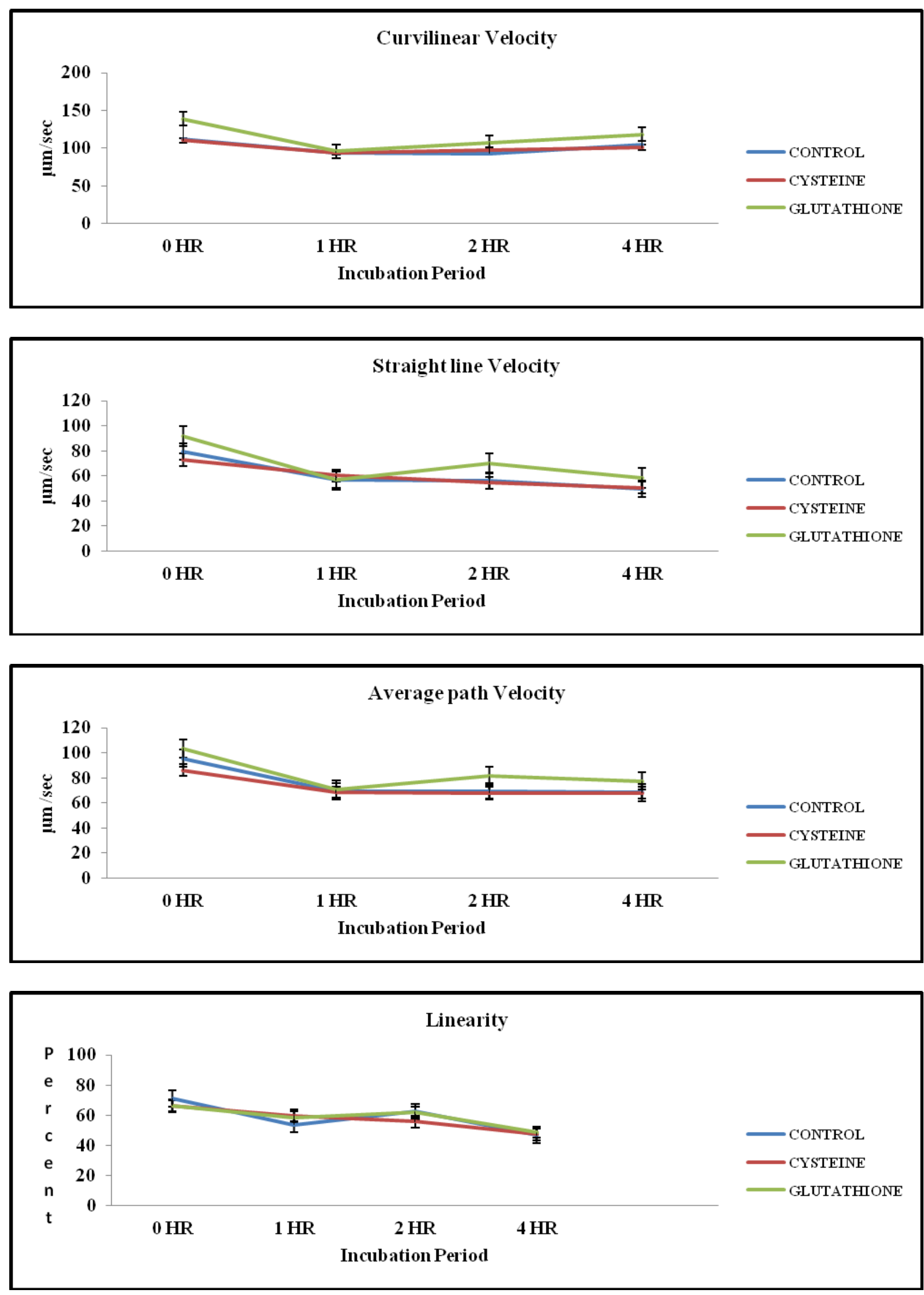

Fig 21a. CASA velocity parameters of control, cysteine hydrochloride and glutathione of poor freezable Jersey crossbred bulls 

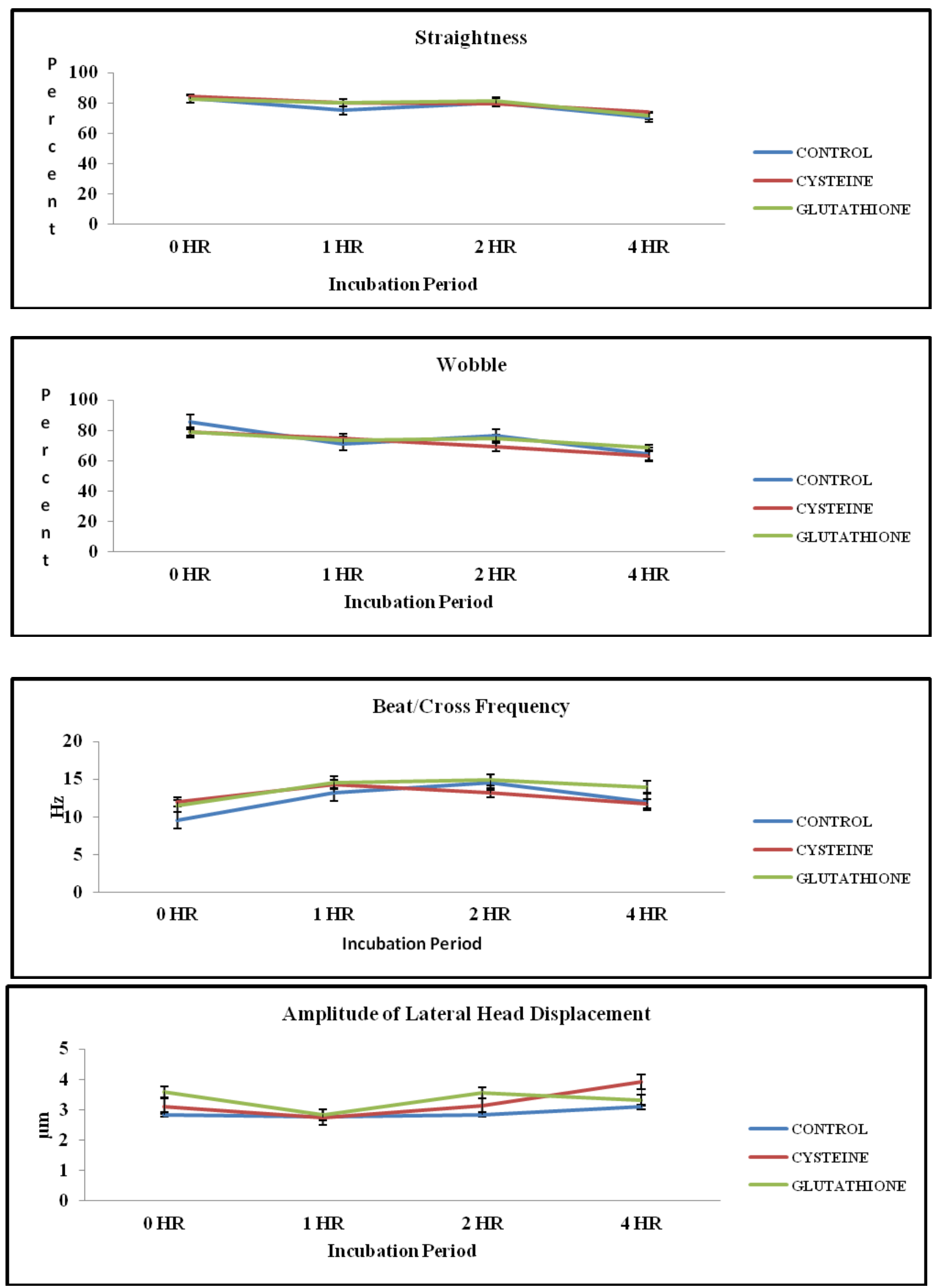

Fig 21b. CASA velocity parameters of control, cysteine hydrochloride and glutathione of poor freezable Jersey crossbred bulls 
TABLE 8. CONCEPTION RATE OF CROSSBRED JERSEY BULLS WITH DIFFERENT SEMEN ADDITIVES

\begin{tabular}{|c|c|c|c|c|c|c|c|}
\hline \multirow[b]{2}{*}{ Freezers } & \multicolumn{2}{|c|}{$\begin{array}{l}\text { Group I } \\
\text { (control) }\end{array}$} & \multicolumn{2}{|c|}{$\begin{array}{c}\text { Group :II } \\
\text { (cysteine HCL) }\end{array}$} & \multicolumn{2}{|c|}{$\begin{array}{c}\text { Group :III } \\
\text { (Glutathione) }\end{array}$} & \multirow{2}{*}{$\mathbf{X}^{2}$} \\
\hline & $\begin{array}{l}\text { No of cows } \\
\text { inseminated }\end{array}$ & $\begin{array}{c}\text { Conception } \\
\text { rate }(\%)\end{array}$ & $\begin{array}{l}\text { No of cows } \\
\text { inseminated }\end{array}$ & $\begin{array}{l}\text { Conception } \\
\text { rate }(\%)\end{array}$ & $\begin{array}{l}\text { No of cows } \\
\text { inseminated }\end{array}$ & $\begin{array}{l}\text { Conception } \\
\text { rate }(\%)\end{array}$ & \\
\hline Good (18) & 29 & $\begin{array}{c}51.00 \\
(15)\end{array}$ & 41 & $\begin{array}{c}60.00 \\
(25)\end{array}$ & 51 & $\begin{array}{l}72.00 \\
(37)\end{array}$ & $7.14^{\mathrm{NS}}$ \\
\hline Poor (18) & 50 & $\begin{array}{c}48.00 \\
(24)\end{array}$ & 44 & $\begin{array}{c}56.00 \\
(25)\end{array}$ & 47 & $\begin{array}{c}59.00 \\
(28)\end{array}$ & $7.56^{\mathrm{NS}}$ \\
\hline Overall & 79 & $\begin{array}{c}49.00 \\
(39)\end{array}$ & 85 & $\begin{array}{c}58.00 \\
(50)\end{array}$ & 98 & $\begin{array}{c}66.00 \\
(65)\end{array}$ & - \\
\hline
\end{tabular}

NS Not Significant

Figures in parenthesis indicate number of ejaculates 


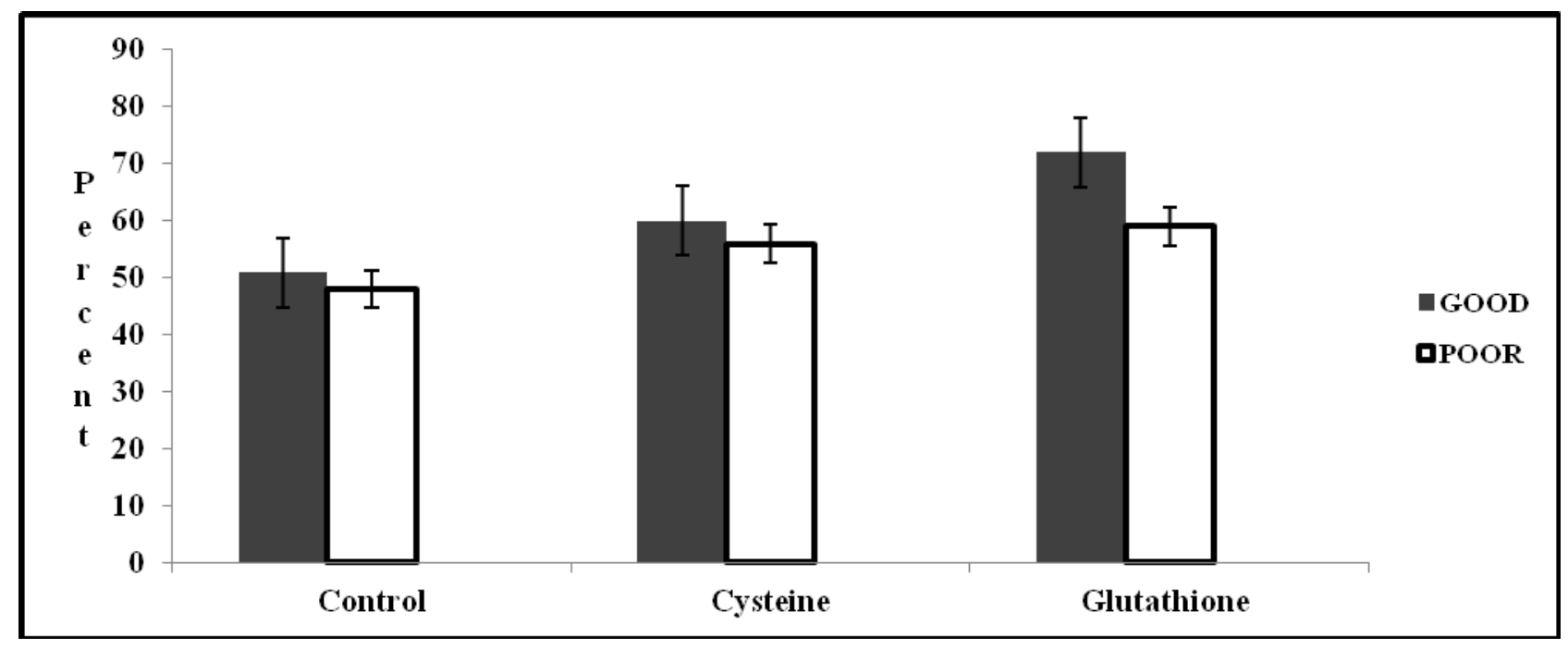

Fig 22. Conception rate of Jersey crossbred bulls with different semen additives

TABLE 9. CORRELATION AMONG SPERM FUNCTIONAL PARAMETERS AND CONCEPTION RATE IN CROSSBRED JERSEY BULLS

\begin{tabular}{|l|l|l|l|}
\hline \multicolumn{1}{|c|}{ Sl. No } & \multicolumn{1}{|c|}{ Parameters } & \multicolumn{1}{c|}{$\begin{array}{c}\text { Conception } \\
\text { rate (r value) }\end{array}$} & \multicolumn{1}{c|}{$\begin{array}{c}\text { Significance } \\
\text { (p) }\end{array}$} \\
\hline 1. & Progressive forward motility & 0.44 & 0.07 \\
\hline 2. & Straight line velocity & 0.70 & $<0.05$ \\
\hline 3. & Curvilinear velocity & 0.52 & $<0.05$ \\
\hline 4. & Average path velocity & 0.51 & $<0.05$ \\
\hline 5. & Plasmalemma integrity & 0.50 & $<0.05$ \\
\hline 6. & Loss of acrosomal integrity & -0.84 & $<0.05$ \\
\hline 7. & $\begin{array}{l}\text { Mitochondrial membrane } \\
\text { potential }\end{array}$ & 0.94 & $<0.05$ \\
\hline 8. & Lipid peroxidation & -0.75 & $<0.05$ \\
\hline
\end{tabular}

(Mean data of all the ejaculates of each group per bull, $\mathrm{n}=18$ were used to calculate the correlation) 


\section{DISCUSSION}

Routine semen analysis is one of the major objectives for assessing the potential ability of breeding bulls. Many of the seminal traits are correlated with conception rate and have got a great importance of selection of bulls for artificial insemination. Adoptable and routine method of semen evaluation mostly comprises microscopical methods like motility assay, Eosin and Nigrosin staining for live and dead, Rose Bengal staining for abnormality, Giemsa and fluorescent staining for acrosomal integrity, nuclear acid fast staining technique and Feulgen's staining technique for examination of DNA integrity and assessment of mitochondrial membrane potential by JC-1 staining and some biochemical tests like lipid peroxide(malondialdehyde) production . Recently many advanced in-vitro methods like Hypo Osmotic Swelling test, Zona free hamster egg penetration test and Cervical mucous penetration test were employed to differentiate comprehensively between fertile and infertile bulls which are correlated with field fertility trials.

Extension of semen with suitable buffer and substrate is an important consideration for prolonged storage and dissemination both at refrigerated and ultra low temperature level. The use of sodium citrate, egg yolk and glycerol has long been established as a potential component for semen preservation (Lardy and Philips, 1941; Salisbury et al., 1941 and Polge, 1949). Reports of addition of various substrates or additives have been highlighted with a view to improve survivability and fertility. Addition of vitamins, hormones, chelating agents and antioxidants to the semen extender has been tried for enhancing the potential fertilizing ability of bull spermatozoa. Cysteine, a sulphur containing amino acid with thiol group is an important component of nucleic acid presents in head of sperm which maintains the integrity of the DNA. This is also an important component of glutathione which acts as an antioxidant. Glutathione not only preserves the anti-oxidant property but also protects the sperm due to its sulfur and cysteine content. In sperm preservation, the formation of peroxides is one of the major drawbacks which affects the fertility and viability of the sperm. So it is of paramount importance that maintenance of optimum semen characteristics during preservation is a pre-requisite for enhanced fertility in cow. In order to ascertain the beneficial effects of additives the present investigation was aimed to determine the effect of additives like glutathione and cysteine hydrochloride and correlate it with certain in-vitro and in-vivo conditions in crossbred bulls. 


\section{SEMINAL ATTRIBUTES OF GOOD AND POOR FREEZABLE CROSSBRED BULLS}

\section{Volume}

The mean average volume for good and poor freezable cross bred Jersey bulls were found to be $6.40 \pm 0.41$ and $5.20 \pm 0.36 \mathrm{ml}$, respectively (Table-1). The present observation is in agreement with Uysal et al. (2007) in the HF Crossbred bulls, Bhoite et al. (2008) in Friesian Gir cross bred bulls and Raina and Dhami, (2004) in Jaffarabadi bulls. A lower value has been reported by Bedi et al. (1984), Pandey and Gupta, (2004) in Crossbred Jersey bulls, where as higher values has been reported by Raina and Dhami, (2004) in Gir bulls. The differences in seminal volume might be attributed to age, genetic make up, nutrition, temperature and individual variation.

\section{Mass Activity}

The overall grading of mass activity of neat semen was $2.50 \pm 0.13$ and $2.40 \pm 0.13$, respectively in good and poor freezable cross bred Jersey bulls (Table 1). However, Erb et al. (1956) demonstrated that it is more closely related with other seminal traits than fertility. The mass motility of spermatozoa is a wholesome effect of sperm concentration and individual motility (Zemjanis, 1970). The present finding has correlated with the findings of Sagdeo et al. (1990) in 75 percent JY, 62.5 percent JY bulls. Higher rating was recorded by Sagdeo et al. (1990) in Pure JY bulls, Raina and Dhami, (2004) in Gir bulls and Munsi et al.(2007) in HF crossbred bull, where as, lower values than the present investigation were also reported by Sagdeo et al. (1990) in 50 percent JY, 75 percent HF and 62.50 percent HF crossbred bulls. In the present investigation, the average rating of mass motility was more than two; hence the extension of semen was tenable.

\section{Sperm Concentration}

The overall concentration of sperm in good and poor freezable crossbred Jersey bulls was $1240.26 \pm 82.77$ and $1007.53 \pm 50.28$ millions per cmm, respectively (Table 1). It was observed from the present investigation that the sperm concentration $\left(10^{6} / \mathrm{cmm}\right)$ ranged from $1062.40 \pm 74.99$ to $1560 \pm 138.69$ in good freezable crossbred Jersey bulls where as in poor freezable bulls it was ranging from $961.00 \pm 91.98$ to $1055.20 \pm 81.14$. The sperm concentration of a fertile bull ranges from 300 to 2000 millions per cmm with an average of 800 millions (Roberts, 1971). Saxena et al. (1978) reported a sperm concentration of 912.33 million per $\mathrm{ml}$ in CB Jersey and recorded a significant correlation with volume. But the present investigation does not indicate any positive corelationship between volume and sperm concentration. The present finding is also comparable with Uysal et al. (2007), Bedi et al. (1984) in Crossbred Jersey bulls, Munsi et al. (2007) in HF Crossbred bull, Pandey and Gupta, (2004) in Crossbred bull semen and Mandal et al. (2005) in 
Sahiwal bulls. The sperm concentration in bovine species varies between breeds, age, climate, testicular diseases, frequency of collection and libido and in many cases there was also individual variation within breed.

\section{Individual Motility}

The individual motile sperm per cent (Table 1) in all the three good and poor freezable experimental bulls registered more than 70 per cent of motile sperm. In the good freezable crossbred Jersey bull, it was $77.13 \pm 0.60$ per cent $(76.20 \pm 0.96$ to $78.00 \pm 1.22)$ where as, in poor freezable semen it was $75.33 \pm 0.77$ per cent $(72.00 \pm 4.80$ to $76.00 \pm 1.58)$. However, Erb et al. (1956) demonstrated that it is more closely related in other seminal traits than fertility and suggested that, the bovine semen with 40 to $50 \%$ motile sperm should be considered as subnormal and was associated with infertility. Blom (1950) also he reported that motility below 50 percent was often associated with low conception rate and poor fertility. Bratton et al. (1951) opined a minimum initial sperm motility of $70 \%$ required for an effective breeding. Similar observation in consistent to present findings have been recorded by Barik et al. (1987) in cross bred bull, Sagdeo et al. (1990) in Pure JY bulls Dhami et al. (1991) and Moharatha, (2001) in Cross Bred Jersey bull. However, higher individual motility per cent has also been observed by Uysal et al. (2007) and Dhami et al. (1991) in pure bred Jersey bulls. Similarly lower individual motility per cent has also been recorded by Bedi et al.(1984), Pandey and Gupta, (2004), Sagdeo et al. (1990) in 75\% JY, 62.5\% JY, 62.5\% HF and 75\% HF crossbred bulls, Chauhan et al., (1993) in 75\% cross bred bull and Mandal et al.(2005) in Sahiwal bulls.

The individual motility is an important parameter for assessment of semen quality and can yield a credible picture of semen potency. Sperm motility is essential during transportation in oviduct and oocyte penetration which can not be ruled out. However a decrease in sperm motility is observed in disease condition, change of environment and temperature variations and it fluctuates between breeds, individuals, age group and technique employed for observation.

\section{Live Sperm}

The live sperm percent in present study was observed to be $89.95 \pm 0.49$ and $85.22 \pm 0.28$ in the good freezable and poor freezable crossbred Jersey bull semen, respectively. The present finding is in consonance with the observation made by Bedi et al., (1984), Barik et al. (1987), Rao and Rao, (1991), Pandey and Gupta, (2004) and Uysal et al. (2007),while lower live sperm per cent has been reported by Raina and Dhami, (2004), Mandal et al. (2005) and Bhoite et al. (2008). The percentage of stained spermatozoa is dependent on various factors like duration, type of diluent 
used, stain integrity and technique employed. Moreover, it gives vital information regarding the livability of both neat and extended semen. The pre freeze semen livability in the present experiment was within the optimum range.

\section{Total Abnormal Spermatozoa}

Overall sperm abnormality percent for good freezable and poor freezable crossbred Jersey bull were recorded to be $6.33 \pm 0.19$ and $9.39 \pm 0.27$, respectively. The present value is correlated with the observation made by Pandey and Gupta, (2004) and Mandal et al. (2005). Lower values were reported by Uysal et al. (2007) and Singh et al. (1997) where as Mandal et al. (2005) and Mathur et al. (1991) reported higher values than the present observation. Roberts (1971) was of opinion that in a fertile semen sample, the total sperm abnormalities should not exceed more than 20 percent. In the present study, the overall sperm abnormality includes acrosomal, caput, mid piece and tail abnormality. Rottenstone and Andersen (1956) concluded that high level of abnormal sperm was not necessarily indicative of low fertility. Moreover, the sperm abnormality may vary due to method of collection, temperature shock (Hancock, 1952) and technique employed (Bishop et al. 1954).

The analysis of variance (Table 2) of seminal traits between good and poor freezable bulls revealed no significant difference with regards to mass activity. Where as other seminal parameters like volume and sperm concentration had a significant difference $(\mathrm{p}<0.05)$, while a high significant difference $(\mathrm{p}<0.01)$ was recorded with respect to individual motility, livability and total sperm abnormality.

\section{Loss of acrosomal integrity}

The maintenance of acrosomal integrity is important for sperm movement and fertilization while preserving the enzyme integrity of sperm head. During the sperm penetration at the time of fertilization the essential enzymes like acrosin, proacrosin and hyaluronidase play an important role for dissolution of membrane layer of an ovum. Hence, any alteration in the structural integrity may lead to loss of these enzymes which ultimately reduces the sperm capability of penetration of oocytes layers leading to fertilization failure. From the present study it was found that loss of acrosomal integrity was higher in poor freezer $(12.73 \pm 1.12)$ than in good freezer $(6.46 \pm 0.90)$ and a highly significant difference $(\mathrm{p}<0.01)$ exists between these two groups. 


\section{POST THAW SEMINAL CHARACTERSTICS}

\section{Motility}

The post thaw motile sperm per cent revealed a highly significant difference $(p<0.01)$ in both the good and poor freezable crossbred bulls (Table 2). In glutathione added group (group III) the motile sperm significantly differed from $(p<0.01)$ group I and group II. Where as the later two groups did not differ significantly in good freezable bull semen. In poor freezable groups the values were significantly different $(\mathrm{p}<0.05)$ from each other. The glutathione added semen showed significantly higher motility value irrespective of groups which indicative of glutathione is being more effective in maintaining higher motility in bovine semen. The present observation of higher motility in glutathione added semen is comparable to the findings of Jain and Arora (1988) and Uysal et al. (2007). However slightly lower value has also been reported by Munsi et al. (2007) and Zekariya Nur et al. (2005), who reported significantly higher motility percent in glutathione added semen in crossbred bulls. It has been observed that a spermatozoon in liquid medium is propelled forward by flagellar beating initiated from distal centriole through electron transport. During ultra low freezing the spermatozoa is exposed to cold shock resulting in damage to the sperm membrane and leakage of enzymes. All these adverse factors might be mitigating by incorporation of glutathione in the semen which act as a membrane stabilizer (Maxwell and Stojanov, 1996).

\section{Livability}

The overall live percentage following freezing in good freezer was highest $(76.28 \pm 0.37)$ in glutathione treated semen (group III) followed by cysteine $(70.85 \pm 0.32)$ and lowest $(63.09 \pm 0.37)$ value was observed in control. Similar trend was also observed in poor freezable bull semen, with highest value in group III (69.32 \pm 0.35$)$ followed by group II (60.92 \pm 0.35$)$ and group I (56.78 \pm $0.56)$.

The present finding is at a with finding of Uysal et al. (2007), Gadea et al. (2005b) and Singh et al. (1997) in EYTG diluter.

During in vitro preservation the spermatozoa are exposed to a foreign diluting media as well as to low and very low temperature. Death might occur due to release of toxic substances, ultra low exposure, enzymatic leakage, medium of preservation, degree of sperm permeability, aging effect of sperm and individual variation (Salisbury et al. 1979). During liquid nitrogen storage, there is a greater chance for death of sperm due to extreme fluctuation in temperature. Moreover, there is an inherent limit that many of the bull spermatozoa could not resist exposure to ultra low temperature. Any of the factor or factors might adversely affect sperm livability. As a whole, the live percentage 
following different application is within the optimum limit and was suitable for artificial insemination. However addition of cysteine or glutathione might have protective action rendering better livability per cent in respective groups (Uysal et al., 2007).

\section{Total Sperm Abnormality}

The sperm morphological abnormalities in all the experimental groups ranged from 9.50 to 13.00 percent and 14.26 to 18.06 percent, respectively consequent to freezing in good and poor freezer (Table 2).

The cysteine added group (group II) did not differ significantly either from group I (Control) and group III (glutathione). However the group I and group II differed significantly $(p<0.01)$ in good freezable bulls. On the contrary in poor freezable bulls all the groups differed significantly from each other $(\mathrm{p}<0.01)$. Roberts $(1971)$ opined that in fertile semen sample, the total sperm abnormality should not exceed more than 20 percent. In the present study the overall sperm abnormality includes caput, mid-piece and tail abnormalities. Rottenstone and Andersen (1956) from their experiment concluded that high level of abnormal sperm was not necessarily indicative of low fertility. Moreover, the sperm abnormality may vary due to method of collection, temperature shock (Hancock, 1952) and technique employed (Bishop et al., 1954). Hence, the difference between the good and poor freezable bull semen may be attributed to these factors.

\section{Loss of acrosomal integrity}

The loss of acrosomal integrity in post freeze state revealed a highly significant difference $(p<0.01)$ among various groups both in good and poor freezers. In good freezers, group III $(8.44 \pm$ $0.63)$ semen deviated significantly $(\mathrm{p}<0.01)$ from group I $(12.20 \pm 0.71)$ and group II (10.56 \pm 0.92), whereas the later groups (group I and II) did not differ. In the poor freezing groups the same trend was followed with a lowest loss in group III (13.01 \pm 0.60$)$ followed by $(15.02 \pm 0.70)$ in group II and $15.97 \pm 0.50$ in group I. The present observation partially corroborates the findings of Uysal et al. (2007). Further, it was evident that addition of glutathione and cysteine in extended semen has significantly reduced the loss of acrosomal integrity in both good and poor freezable bulls under observation.

Bull semen diluents with glutathione incorporation have been reported to improve post thaw motility and normal acrosome. It has also been reported to reduce enzyme leakage and consistently improved fertility. The mode of action as suggested might be due to limited the production of lipid peroxides (Satish Kumar, 2007). 
The physiologic acrosome reaction is a well-coordinated process that can occur only in a living spermatozoan in response to natural inducers. In contrast, loss of acrosomal content can occur with the breakdown of the membranes during cell death, cryopreservation and during addition of oxidants which shows similar acrosomal changes (Bedford, 1970).

\section{Hypo-Osmotic Swelling Test (HOST)}

The post freeze value of HOS positive sperm revealed a significant difference $(\mathrm{p}<0.01)$ both in good and poor freezing group. In good freezer all the three groups differed significantly ( $p$ $<0.01)$ from each other with highest value obtained in group III $(81.19 \pm 0.30)$ followed by group II $(70.47 \pm 0.35)$ and group I $(65.74 \pm 0.27)$. In poor freezing group the group III revealed higher value $(71.29 \pm 0.26)$ which differed from group I $(57.03 \pm 0.32)$ and group II $(57.65 \pm 0.47)$, whereas no significant difference exists between the later groups (Table 3).

These observations indicated that addition of glutathione and cysteine in the extended semen has significantly elevated the HOST value than the control. Uysal et al. (2007) recorded $79.3 \pm 3.7$ of total swollen sperms in glutathione treated semen in bulls following HOST. Nair (1997) found post thaw HOS positive value for sperms to be $23.54 \pm 1.41$ for half bred and $23.83 \pm 1.2$ in 3 bred cross bulls with a significant $(\mathrm{P}<0.05)$ difference between breeds. Moharatha $(2001)$ reported the overall per cent of HOS positive sperm to be $33.50 \pm 0.74$ (28.40 to 37.80) in crossbred Jersey semen following cryo preservation. The post freezing analysis also partially corroborates the finding of Uysal et al. (2007).

Routine semen evaluation has certain limitations for comprehensive prediction of fertility of bull semen. The HOST highlights the permeability of sperm membrane to Hypo osmotic solution and the projection of higher value is a valid indication of intact membrane and sample with higher value is regarded as potent for establishing pregnancy.

The comparatively higher value in group II and III with respect to HOST could be attributed to incorporation of biochemically active substance (cysteine) or due to presence of antioxidant (glutathione). As no relevant information was available regarding these semen additives, it was not possible to substantiate the present finding conclusively. The individual and overall values of good freezer were higher than the poor freezer and the glutathione added semen have shown higher value than cysteine and control group. This indicates that the glutathione additives have capacity to maintain the membrane integrity better than the other additives and control group. 


\section{Cervical Mucus Penetration Test (CMPT)}

The in-vitro sperm penetration test is considered as a sperm function test that measures the ability of sperm in the semen to swim up into a column of cervical mucus or its substitute. The sperm migration into the cervical mucus or its substitute is based on the same principle as per the test proposed by Kremer et al. (1995).

Bovine cervical mucus is particularly common during estrous and it is easier to obtain large quantities with similar rheological and biochemical properties to human cervical mucus which can be stored in frozen storage with only minimal changes in rheological properties (Lee et al., 1981). So CMPT has always considered as an important in-vitro sperm function test in bovines. This method in conjunction with HOST can give a fair assessment of semen viability for selection of motile and morphological normal sperm for fertilization. The present study revealed that semen extended with glutathione incorporation, showed highest percent of cervical mucus penetration followed by cysteine and control group. Similar trend of mean vanguard distance traveled by the sperm were noted to be highest in group III followed by group II and group I. The present investigation also demonstrated that, extended semen when added to glutathione, a significant difference in millimeter of penetration to the cervical mucus occurred as compared to other two groups. This significant difference can be attributed to the fact that the migration of the vanguard distance and swim up of sperm velocity were higher due to enhanced straight forward motility as adequate motility has been ascribed to normally produce adequate mucus penetration (Takemota et al., 1985). Moreover, ability of spermatozoa to penetrate bovine cervical mucus was found to be dependent on various semen parameters which could not be accurately predicted on the basis of single test.

David et al. (1979) were of opinion that intrinsic sperm motility test is a crucial property for penetration of cervical mucus. This is supported by the present findings that the percent of motile spermatozoa in glutathione added extender can be best correlated with cervical mucus penetration test. However sample showing good motility but poor in penetration may indicate other factors such as morphology which are also important for mucus penetration. The bovine cervical mucus plays an important role in selecting motile, morphological normal sperm for fertilization. So CMPT has always been held as a potential and important in-vitro sperm function test.

The present findings corborate with observation made by Bhatia, et al. (2007) that fresh semen had the maximum sperm penetration in bovine cervical mucus followed by extended and frozen semen $(38.63 \pm 0.26$ to $36.28 \pm 0.81)$. This indicated that with higher sperm penetration distance there is an increase in percent of the animal to conceive for particular bull semen. The 
individual variation between the bulls in good freezer and poor freezer semen could be attributed to the fact that the sperm penetration speed was significantly different between the bulls as well in between the mucus samples as proposed by Anil kumar and Devanathan (1995).

The difference of cervical mucus penetration with regard to the semen of good freezable and poor freezable crossbred bulls would possible due to the difference in the post thaw motility, abnormality and loss of mitochondrial membrane potential, which could otherwise has resulted in lowering the penetration rate. As such glutathione incorporation to the extended semen might have potentiated the viability of the sperm with respect to its motility and morphological integrity which was reflected by the highest rate of penetration through the cervical mucus in the present course of investigation. Hence, this test can indicate the sperm function during the later stages of its use and can reflect in the conception rate of the animals Bolarndeola et al. (2003) opined hat $\mathrm{SMPT}_{\mathrm{vd}}$ has a low accuracy in evaluation of semen but $\mathrm{SMPT}_{\mathrm{sc}}$ was found to be moderately accurate for assessing sperm motility in semen. Therefore, method of using sperm concentration, instead of vanguard distance, as diagnostic criteria of in-vitro SMPT has potential as a useful laboratory based sperm function test. However, Steven (2004) observed that despite the relatively high repetition test no relation was found between sperm migration distance and the non-return rates.

The present observation finds the supports of Satish Kumar and Kadirvel (2007) who observed that the frozen thawed spermatozoa covered a significantly high distance in cervical mucus than that of fresh spermatozoa. Thus indicating it is to be a good indicator for predicting the functional status of frozen spermatozoa. However, lower rate of sperm penetration has been reported by Kumaresan et al. (2005), while comparing the pre freeze and post thawed bovine cervical mucus test in non-luteal oviductal protein luteal oviductal protein and control.

\section{DNA fragmentation}

The process of spermatogenesis involves the reorganization of chromatin materials in developing spermatids through protamines. In-vivo study revealed that protamine condenses the DNA of vertebrate sperm cells in to thousands of particle that vary in diameters from $50-100 \mathrm{~nm}$. Bull protamine has been found to condense DNA in to spherical particle with the range of protamine-DNA condensates observed in bull spermatozoa cell nuclei.

Saacke et al. (1998) observed that sperm with non-compensable defects such as abnormal DNA distribution has been associated with improper zygotic, embryonic and or fetal development. Thus, any abnormality associated with DNA, chromatin packing, or the sperm nuclear matrix 
should be reflected by a change in sperm nuclear shape. The evaluation of sperm DNA distribution should be useful for assessing male fertility (Vilfan et al., 2004).

The present investigation revealed the mean sperm DNA fragmentation in good freezable bulls was highest in control with $(10.77 \pm 0.67)$ followed by group II $(8.83 \pm 1.13)$ and least in glutathione treated group $(6.94 \pm 1.28)$. A significant difference existed between the control and glutathione group, whereas glutathione and cysteine added group did not exhibit any difference with regard to percent of DNA fragmentation. However, analysis of variance revealed a high significant difference $(\mathrm{p}<0.01)$ between all the three groups under all observations. The study indicates that addition of glutathione to the extended semen potentiates the metabolic regulation due to presence of sulpha-hydryl group. The low DNA damage observed following supplementation with GSH suggest that ROS generation and redox balance are the most important factors responsible for disruption and stability of sperm chromatin after cryopreservation (Joaqu1, et al., 2007).

In poor freezable bulls it was observed that there was sharp increase in the per cent of DNA fragmentation from $9.98 \pm 1.17$ in glutathione added semen to $24.70 \pm 1.16$ in control group with the cysteine incorporated group showing the percent of fragmented DNA to be $16.42 \pm 2.19$ per cent.

Evenson and Wixon (2006) found evidence that semen samples containing a statistical threshold of $\geq 30 \%$ sperm DNA fragmentation have a reduced level of pregnancy success and it was suggested that DNA fragmentation is due to an apoptotic event or to reactive oxygen species (ROS).

Perusal of literature did not reveal study on DNA fragmentation in good and poor freezable bulls after incorporation of glutathione or cysteine. So the appropriate comparison could not be substituted by this study.

Uysal, et al. (2007) described that the free radicals are known to be involved in lipid peroxidation as well as DNA and sperm membrane damages which may lead to decreased sperm motility or cell death. Hence, in the present study the lower percent DNA damage in the glutathione added semen might be a beneficial factor in avoiding the process of damage and reduce generation of ROS which would otherwise have negatively affected the fertilization rate as evidenced from field fertility trial.

Słowińska et al. (2008) observed that cryopreservation caused a significant but low (3.8\%) decrease in the percentage of DNA in the comet head and an increase (5.3\%) in the tail length. This indicates that in addition to motility and viability, low levels of DNA fragmentation after cryopreservation is a characteristic of bull spermatozoa and can be a part of remarkable cryoresistance of bull spermatozoa by glutathione through the action of sulpha-hydryl group. There 
is also beneficial effect of glutathione through protection of DNA integrity maintenance in sperm cells by the action of its anti-oxidant property and also the content of cysteine to aid into the synthesis of protamine that might be responsible for prevention of DNA fragmentation in sperm cells.

Cotran et al. (1989) observed that toxic oxygen metabolite is emerging as a final common path way of cell injury as well as cellular ageing. So the anti-oxidant enzymes like glutathione peroxidase destroy the free radicals if there is damage to the cell membrane by lipid peroxidation. Hence, the role of glutathione can not be undermined in augmenting the role of scavenging reactive oxygen, intermediate and other free radicals such as $\mathrm{H}_{2} \mathrm{O}_{2}$ with help of glutathione reductase. Therefore it was quite reasonable to accept that glutathione might protect the spermatozoa membrane damage by inhibiting the lipid peroxidation process. This is in accordance with observation made by Foote et al. (2002) that spermatozoa are weak anti-oxidant and there by undergoes lipid peroxidation in the presence of oxygen. Addition of anti-oxidants like glutathione might have beneficial effect on sperm particularly in media, devoid of macromolecule for several hours. This beneficial effect during pre-freezing process might have some beneficial carryover effect on sperm through evaluation of sperm motility, abnormality and other semen analysis.

\section{Lipid Peroxidation (LPO)}

During preservation or cryopreservation the semen is exposed to cold shock at atmospheric oxygen which in turn increases the susceptibility to lipid peroxidation due to higher production of reactive oxygen species. Thus, it induces ageing of sperm thereby reduces the life span and affects the preservation of semen for AI with poor success rate. The lipid peroxidation occurring in seminal plasma and spermatozoa can effectively be measured by MDA concentration.

The present investigation revealed that the MDA production ( $\mathrm{nmol} / 10^{8}$ sperms) was estimated to be lower in group III $((2.17 \pm 0.82)$ while increasing to $(2.60 \pm 0.76)$ in group II and $(2.73 \pm 0.85)$ in group $\mathrm{I}$. The difference in the concentration was found to be non-significant in all the groups in good freezable bull semen. The corresponding value in poor freezable bull semen was observed to be $3.17 \pm 1.34,3.50 \pm 1.42$ and $3.68 \pm 1.65$ respectively. From the present study it was evident that through the production of MDA increased from group III to group I, the difference was non- significant. Numerically, group III bull semen, where glutathione was added during the semen extension, the production of MDA was lowest. This is indicative of the better action exerted by antioxidants like glutathione where the lipid peroxidation free radicles like $\mathrm{H}_{2} \mathrm{O}_{2}$, super oxide anion, and hydroxy radicals have been combated leading to prevention of structural damage of sperm membrane during freezing and thawing process. Thus, glutathione might have played an important 
role in inhibiting the detrimental effect of lipid peroxidation. So the process of anaerobic glycolysis, and synthesis of DNA, RNA and protein synthesis would have functioned better in the glutathione added group as compared to control group.

\section{Mitochondrial membrane potential (MMP)}

The mitochondria of the sperm play an important role in energy production for migration in the form of ATP. The mitochondria are also made up of membrane and other cellular structure and also suffer from all effects produced by the lipid peroxides. Mitochondria are the major organelles for the production of adenosine triphosphate (ATP) which is very important for maintaining flagellar movement for a long period of time. This is required for sperm to reach the site of fertilization (Bartoov et al., 1980). So measurement of mitochondrial membrane potential gives an idea about the fertility status of the sperm indirectly. Motile sperm stained with JC-1 appeared orange in the mid-piece indicating a high mitochondrial membrane potential whereas immotile sperm with a low membrane potential stained green. The percentage of spermatozoa staining orange was highly correlated with expected sperm viability (Curtis et al., 2000).

The high mitochondrial membrane potential was correlated with fertility as compared to reactive oxygen species production, DNA fragmentation and viability (Marchetti et al., 2002).

Motility is currently the most commonly used method to evaluate sperm quality in commercial bull stations. At best it is an indicator or an indirect measure of metabolic activity and cell viability which indirectly depend upon the MMP (Hallap et al., 2005).

In the present investigation the semen of good and poor freezer which was treated with cysteine and glutathione recorded better results as compared to the control group. The analysis of variance revealed no significant difference between the treatment group and control group, which coroborate with the findings of Gadea et al. (2005), who also reported that when the MMP increases the motility parameters and fertility potential also increases. Chen et al. (1989) also reported that a change in MMP is a good indicator of sperm functional status.

The formation of aggregates depends on the chemical environment of dye concentration, $\mathrm{pH}$, ionic strength, and temperature. Fluorescent mitochondria may not have been the sole indicator of sperm motility. Thus, it may be possible that mitochondrial fluorescence quenched or failed to fluoresce at very low levels while the spermatozoa continued to exhibit relatively slower progressive motility. This biological significance of the proportions of aggregates and monomers in spermatozoa remains to be resolved. 
Mitochondrial Membrane Potential is widely used for characterization of cellular metabolism, viability and apoptosis in various cellular models. Wang et al. (2003) found a correlation between poor sperm mitochondrial function and diminished motility and reduced fertility in humans. It has been suggested that mitochondria, acrosome and plasmalemma of unfrozen spermatozoa vary in their response to the addition of the cryoprotectant glycerol (Garner et al., 1999).

Comparison of sperm mitochondrial transmembrane electric potential as reflected by gradient of red-orange to green fluoresces, from extended semen with glycerol and additives like cysteine and glutathione showed a shift from high to low transmembrane electrical potential. The non-significant difference obtained in the good and poor freezable crossbred Jersey bull semen with or without additives might suggest that glycerol though appeared to provide a protective effect to the acrosomal membrane but has got no overall effect on the plasma membrane (Garner et al., 1999)

\section{Incubation Test}

Artificial insemination is an important tool to improve the genetic gain of the progeny which indirectly related to the production process of cow. In AI the frozen semen is usually deposited either in the mid cervix or body of uterus and the sperm will take time to reach the fertilization site (ampulla isthmus junction). In the incubation test, the spermatozoa movement is measured on the basis of forward progressive motility (FPM), non-progressive motility (NPM), total motility and static sperms by CASA technique.

Various motility parameters at different hour storage assessed in good and poor freezer bulls (Table 5) revealed a significant difference $(p<0.05)$ at 0 hour with respect to forward progressive motility per cent. On the contrary other motility parameters like non-progressive motility, total motility and total static sperm percent did not record any significant difference. Perusal of $1 \mathrm{hr}$ incubation values between good and poor freezer showed a highly significant difference $(\mathrm{p}<0.01)$ with respect to forward progressive motility and non-progressive motility. However following 2 hours and 4 hours incubation forward progressive motility differed significantly between good and poor freezer on both occasion of incubation. The total motility percent was significantly higher $(\mathrm{p}<$ 0.05) in good freezing bulls when compared to its counterpart. Similarly static sperm per cent showed a significantly higher value $(\mathrm{p}<0.01)$ in poor freezing bull as compared to good freezer.

Forward progressive motility of spermatozoa is a vital index which is essential during cervical transportation, sojourn through fallopian tube, migration from sperm reservoir and oocyte 
penetration (Hafez, 1987). However, the other indices pertaining to velocity may not be pertinent for accomplishment of such physiological events. In routine semen analysis, microscopic account of straight forward progressive motility is considered to be an important criterion for semen evaluation which is more or less comprehensively assessed for the physiological characterstics of the spermatozoa (Salisbury et al., 1985). A positive correlation between forward progressive motility value with prospective pregnancy have been suggested which has a significant bearing for judging the fertility potential of the male (Maule, 1962).

\section{Effects of additives on sperm motility (CASA)}

The effects of various semen additives in good and poor freezer bull semen have been analyzed with respect to various motility parameters such as progressive forward motility, non progressive motility, total motility and total static sperms. The post thaw progressive forward motility at 0 hours of storage in good and poor freezer bull semen were 69.17 $\pm 4.96,57.08 \pm$ $5.32 ; 69.76 \pm 3.23,55.04 \pm 4.76$ and $71.47 \pm 8.40,58.02 \pm 4.13$, respectively in control (group I), cysteine (group II) and glutathione (group III) added semen (Table 6).Comparison of progressive forward motility and total motility values in various experimental groups showed significantly higher value. In cysteine added semen with respect to forward progressive motility and total motility no much difference could be found for group I and group III at $0 \mathrm{hrs}$ of incubation (Table 6). After 1 hour of storage the corresponding values were $65.38 \pm 4.04,51.03 \pm 7.09 ; 66.43 \pm 7.21$, $44.14 \pm 7.75$ and $66.05 \pm 1.05$ and $53.10 \pm 3.99$ in group I, II and group III respectively. Maintaining a similar trend the cysteine hydrochloride added group (group II) also registered a significantly higher value $(\mathrm{p}<0.05)$ for forward and total motility and the same combinations in group I and group III did not project any significant difference.

Following 2 hours incubation, the progressive forward motility were $52.86 \pm 6.20$ and 43.77 \pm 4.73 in group I, $49.13 \pm 7.14$ and $46.63 \pm 4.25$ in group II and $60.38 \pm 6.62$ and $41.24 \pm 5.90$ in group III for good and poor freezer bull semen. While comparison of good and poor freezing semen, it was revealed that the forward progressive motility and total motility values were significantly higher $(\mathrm{p}<0.05)$ between good and poor freezer bulls. However no significant difference could be measured in group I and Group II treatment for these parameters.

The progressive forward motility values for good and poor freezing bulls were $39.98 \pm 1.06$, $25.09 \pm 3.99 ; 42.96 \pm 5.60,29.77 \pm 3.69$ and $46.98 \pm 8.87$ and $30.79 \pm 1.09$ in group I, group II and group III, respectively. In control group (group I) the forward progressive motility and total motility values were significantly higher $(p<0.05)$ in good freezing bull semen compared to their poor 
freezing counter part. On the other hand no such difference was discernable in group II and group III for such parameters following 4 hours of incubation.

Perusal of incubation data between various groups revealed, consistently higher value at different hours of storage time with respect to progressive forward motility and this implied that glutathione might have played a critical role to augment intracellular defense mechanism against oxidative stress, protecting from the hazards of ice crystals formation during ultra low storage. This might have contributed for maintaining better progressive forward motility during incubation at different hours. This has been amply corroborated by earlier workers (Macleod and Irvine, 1995) suggesting improvement in seminal characterstics. Although much difference in motility parameters could not be accounted between group I and group II for these parameters, but, the cysteine added group showed numerically higher values at 4 hours of storage. In contradiction to this fact many observers have reported better seminal traits with incorporation of cysteine in comparison to routine tris diluent (Alexei et al. 2005). However no such credible difference could be visualized between control and cysteine hydrochloride added group and both performed reasonably for these parameters at ultra low preservation.

\section{Computer Assisted Semen Analysis of post thaw mobility parameters}

The sperm mobility phenotype can be attributed to specific sperm velocity parameters of individual sperm as determined by CASA. The motion parameters VSL, LIN and BCF contribute to the overall sperm mobility phenotype in bulls, as these were all significantly correlated with sperm mobility. In the present experiment various types of sperm mobility representing Curvilinear Velocity (VCL), Straight line Velocity (VSL), Average path Velocity (VAP), Linearity (LIN), Straightness (STR), Wobble (WOB), Amplitude of Lateral Head displacement (ALH), Beat/Cross Frequency (BCF) and others have been displayed in Table 7. The parameters VSL, VAP, VCL, LIN, and BCF were also significantly higher for the males classified as high mobility as compared with the low-mobility males. The parameter LIN is a measure of linearity, and the BCF motion parameter indicates the number of times the sperm track crosses the smoothed path, both of which indicate linear progression. Thus, high-mobility sperm swim faster and straighter than did lowmobility sperm. This may be biologically significant because the sperm mobility phenotype, on the basis of research with whole ejaculates, is predictive of fertility (Froman and Feltmann, 1998 and Froman et al, 1999).

At 0 hour incubation in group III both good and poor freezer showed higher value with respect to VCL. The VSL and VAP values were almost similar in group I and group III. The linearity value was above 66 percent in all groups. In group II and III the BCF values was slightly 
higher than the control group. After 1 hour of incubation group II (cysteine hydrochloride) of good freezer was reflecting superior to all other groups with respect to VCL, VSL and VAP. The linearity values also shared a similar trend in good and poor freezable semen. Consequent to 2 hours incubation, the group III fared better compared to group I and group II with respect to different types of velocity parameters. The linearity value was above 60 per cent in all groups except poor freezable semen of group II. The BCF more or less projected a similar value in all the groups. At the end of 4 hours incubation, the glutathione added group (group III) of both good and poor freezing semen showed consistently higher value in all the velocity parameters compared to their respective counter part in group I and group III. However cysteine added group had a slight edge over group I with respect to both good and poor freezable bull semen. The linearity per cent was highest in group II with respect to good freezer. The BCF remained almost within a similar value.

The present data on motility parameters revealed that the glutathione added group reflected a comparatively higher value consequent to 2 hours and 4 hours incubation in good freezable bulls compared to group I and group II. Similarly in poor freezable bulls' semen of group III also fared better against their group I and II. Following 4 hours of incubation in the group II, both good and poor freezers showed higher value corresponding to the group I.

Recent findings suggested that assessment of motile spermatozoa in a semen sample may not be considered as a reliable index for semen evaluation. The objective and quantitative measurement of other sperm motion characterstics derived from observations of individual cells assessed by CASA have been found to be more efficient in predicting semen sample's potential fertility (Mortimer, 1994). In addition to the use of computerized technique to predict semen fertility, CASA can be an useful tool to study the effects of various in-vitro procedures on sperm motility as well as phenomenon of sperm hyperactivation (Farrell et al. 1993).

\section{Conception Rate}

The conception rate obtained from field fertility trial revealed the highest conception rate of 72.00 per cent in glutathione added semen (group III) followed by cysteine 60 per cent ( group II) and 51 per cent ( group I) out of 51, 41 and 29 animals served artificially by good freezable bull semen. The corresponding values for pregnancy in poor freezable semen were 59 (28), 56 (25) and 48 (24) per cent out of 47, 44 and 50 inseminations. The overall conception rate in different additive groups revealed a highest value of 66 percent (65) and lowest value of 49 per cent (39) from 98 and 79 animals bred artificially. The cysteine added group established a pregnancy of 58 percent (50) from 85 inseminations. The chi-square value did not reveal any significant difference between various experimental groups irrespective of quality of semen used. 
The overall value of conception rate in group III was highest and both good and poor freezable bull semen fared better than their respective counter part in group I and group II. However, the cysteine added group showed better conception rate in both good and poor freezer than their reciprocal groups in controls.

From various studies, glutathione proved to have a better protective value in containing the various hazards of sperm damage during extension and consequent preservation by refrigeration and ultralow temperature (Joaqui et al. 2007). Earlier studies suggested that addition of glutathione improves the number of viable spermatozoa, reduction in ROS generation, lower chromatin condensation, DNA fragmentation, higher oocyte penetration along with improvement in in-vitro embryo production compared to control group (Joaqu et al. 2007). The non significant increase in pregnancy rate in glutathione compared to other treatment groups has all beneficial effect of glutathione as a semen additive.

Addition of cysteine hydrochloride both in good and poor freezable semen also proved to be better than control group. Cysteine is an essential sulphur containing amino acid, which is proved to be a membrane stabilizing agent and ameliorates the metabolic by product like $\mathrm{H}_{2} \mathrm{O}_{2}$ of spermatozoa during sperm motility. Besides resolving intra cellular damage, the moderate improvement in the conception rate against control group may endorse to the fact cited above. However the computer analysis of post thaw frozen semen suggested that mobility and velocity parameters showed better rating in glutathione and cysteine added semen, which also indirectly implied fertility in-vitro. 


\section{SUMMARY}

The present experiment was under taken in the Department of Animal Reproduction, Gynaecology and Obstetrics, College of Veterinary Science and Animal Husbandry, Bhubaneswar in collaboration with Frozen Semen Bank, Cuttack. The objective of the study was to determine the efficacy of Cysteine Hydrochloride and Glutathione as additives in routine frozen semen extender (EYTG) at the rate of $5 \mathrm{mM}$.

Various seminal traits i.e. volume, mass activity, individual motility, sperm concentration, total sperm abnormality, loss of acrosomal integrity and HOST were analyzed in extended semen containing cysteine (group II), or glutathione (group III) with routine control (group I). Post thaw seminal attributes relating to motility, livability, total sperm abnormality, loss of acrosomal integrity, cervical mucus penetration test, estimation of MDA concentration, hypo-osmotic swelling positive sperm per cent and mitochondrial membrane potential DNA fragmentation test were assessed in various experimental groups. Conception rate of various experimental groups were evaluated on the basis of field fertility trial.

Six ejaculates of each good and poor freezable crossbred bull were collected. The average volume was $6.40 \pm 0.41 \mathrm{ml}$ and $5.20 \pm 0.36 \mathrm{ml}$ respectively in good and poor freezable crossbred bull. The mass activity of good and poor freezable cross bred bulls was more or less similar with figures of $2.50 \pm 0.13$ and $2.40 \pm 0.13$, respectively. The average sperm concentration $\left(\mathrm{X} 10^{6}\right)$ per $\mathrm{ml}$ was $1240.26 \pm 82.77$ and $1007.53 \pm 50.28$ in good and poor freezable crossbred bull semen respectively. The corresponding values for motility percent were $77.13 \pm 0.60$ and $75.33 \pm 0.77$ respectively. On an average, the live sperm per cent was more than $85 \%$ for both good and poor freezable semen. The per cent of total abnormal sperm was $6.33 \pm 0.19$ and $9.39 \pm 0.27$ respectively in good and poor freezable bulls. The loss of acrosomal integrity per cent was higher (12.73 \pm 1.12$)$ in poor and lower $(6.46 \pm 0.90)$ in good freezable bulls. The hypo-osmotic swelling positive spermatozoa per cent was higher $(85.78 \pm 0.42)$ in good and lower $(80.38 \pm 0.32)$ in poor freezable bulls. The seminal parameters like individual motility, live sperm percent, total sperm abnormality, loss of acrosomal integrity and HOST showed highly significant difference $(p<0.01)$ between the groups. The other parameters like volume and sperm concentration also differed significantly ( $\mathrm{p}<$ $0.05)$.

The individual motility of post freeze semen showed more than $50 \%$ overall value irrespective of extender used, for all the experimental bulls. The good freezable crossbred bull had showed higher motility percent than poor freezable crossbred bulls, irrespective of additives used. But the glutathione added group showed highest motility of $74.12 \pm 0.39$ and it was lowest $(61.83 \pm$ 
0.48) in cysteine hydrochloride added semen in good freezable bulls. Similarly the glutathione extended semen also showed same trend in poor freezable bull $(66.83 \pm 0.42)$. The overall average motility per cent of group I, II and III were also more than 50\%. Comparison of individual motility value in experimental groups recorded highly significant difference $(\mathrm{p}<0.01)$ among three groups for both the good and poor freezable crossbred bulls.

The post freeze live sperm percent was observed in glutathione (group III) and cysteine (group II) treated group of good freezer with the value of $76.28 \pm 0.37$ and $70.85 \pm 0.32$ while in control group (group I), it was $63.09 \pm 0.37$. In the poor freezer, it was $69.32 \pm 0.35,60.92 \pm 0.89$ and $54.32 \pm 0.24$, respectively. Analysis of variance revealed a high significant difference $(p<$ 0.01 ) in treatment and control groups in both good and poor freezable semen.

The total sperm abnormality percent in good freezer revealed almost similar value for group III $(9.45 \pm 0.94)$ followed by group II $(11.42 \pm 0.79)$ and group I $(13.18 \pm 1.41)$. The similar trend was found in poor freezer with a value of $14.26 \pm 0.18,15.87 \pm 0.26$ and $18.06 \pm 0.18$ respectively while both the classes showed highly significant difference $(p<0.01)$ among groups.

The loss of acrosomal integrity for group III was $8.44 \pm 0.63$ and $13.01 \pm 0.60$ for good and poor freezer respectively, which was significantly different $(p<0.01)$ from rest of the groups in respective category. On the contrary, group II and group I value did not differ significantly.

The post thaw value of HOST per cent in good freezer bulls was $65.74 \pm 0.77,70.47 \pm 0.35$ and $81.19 \pm 0.30$ in groups I, II and III respectively. The corresponding values for poor freezable bulls were $57.03 \pm 0.32,57.65 \pm 0.47$ and $71.29 \pm 0.26$, respectively. Exhibition of highly significant difference $(\mathrm{p}<0.01)$ was observed among groups in both good and poor freezers. The glutathione added group showed significantly higher rating for HOST with respect to freezing type.

The vanguard distance $(\mathrm{mm})$ travelled by the sperm in bovine cervical mucus after thawing was higher in glutathione treated group than the other group in both good $(31.16 \pm 1.09)$ and poor $(25.44 \pm 1.35)$ freezable crossbred bulls. The cysteine hydrochloride treated group revealed second highest value next to the former in both good $(27.76 \pm 0.98)$ and poor $(23.35 \pm 1.18)$ freezable bulls. The control untreated group recorded lowest value $(25.84 \pm 1.35$ and $20.24 \pm 1.09)$ as compared to former groups.

DNA fragmentation of post thawed semen revealed that untreated control $(10.77 \pm 0.67$ and $24.70 \pm 1.16$ ) group had higher percent of fragmentation in both good and poor freezer as compared to cysteine $(8.83 \pm 1.13$ and $16.42 \pm 2.19)$ and glutathione $(6.94 \pm 1.28$ and $9.98 \pm 1.17)$ treated groups. 
The MDA production (nmol/10 ${ }^{8}$ sperms) of post thaw semen in group III $(2.17 \pm 0.82$ and $3.17 \pm 1.34)$ had lower value in both good and poor freezable crossbred bull as compared to group II $(2.60 \pm 0.76$ and $3.50 \pm 1.42)$ and control $(2.73 \pm 0.85$ and $3.68 \pm 1.65)$.

The percent of mitochondrial membrane potential positive sperm of post thaw semen revealed that glutathione $(29.78 \pm 0.98$ and $27.55 \pm 1.64)$ treated group had higher value in both good and poor freezable crossbred bull as compared to cysteine $(28.63 \pm 3.08$ and $26.11 \pm 2.96)$ treated and control $(26.11 \pm 3.27$ and $25.93 \pm 1.60)$.

Assessment of motility at different hours of incubation in good and poor freezable crossbred bull semen by CASA revealed that the forward progressive motility was higher in good freezer from zero to four hours of incubation than poor freezer. Analysis of variance has also revealed that highly significant difference $(\mathrm{p}<0.01)$ existing at $1^{\text {st }}$ hour and significance difference at 0,2 and 4 hours of incubation. With regards to non- progressive motility the poor freezer found showing value similar to good freezer at all hours of incubation. Analysis of variance had revealed that highly significant $(\mathrm{p}<0.01)$ difference is existing at $1^{\text {st }}$ hour and no significance difference at 0,2 and 4 hours of incubation. Assessment of total motility has also behaved like forward progressive motility. The good freezer has higher value as compared to poor freezer at all hours of incubation period. Analysis of variance had revealed significance difference $(\mathrm{p}<0.05)$ at $4^{\text {th }}$ hour and no significance difference at 0,1 and 2 hours of incubation. As compared to different hours of incubation, the forward progressive motility and total motility have been lowered from 0 hours to 4 hours, but the non- progressive motility and static sperm percent were found increasing gradually from 0 hours to 4 hours of incubation.

Comparison of motility between control and treatment groups at different hours of incubation has revealed that the forward progressive motility, non - progressive motility and total motility was higher in glutathione treated group at different hours of incubation period than cysteine treated and untreated control group. Contrastingly the total static sperm percent was higher in control group than either of glutathione or cysteine treated group. Analysis of variance revealed that the forward progressive motility, non - progressive motility and total motility were showing highly significant difference $(\mathrm{p}<0.01)$ at 1 hour incubation period and there was no significant difference at 0,2 and 4 hours incubation periods. Total static sperms were not showing any significant difference at different hours of incubation.

Computer assisted semen analysis (CASA) of some post-thaw seminal characteristics in control and treatment groups at different hours of incubation has revealed that the CASA parameters like curvilinear velocity, straight line velocity, average path velocity, linearity, 
straightness, wobble, amplitude of lateral head displacement and beat/cross frequency were higher in good freezer as compared to poor freezer at different hours of incubation and glutathione have higher value than other treatment and control groups in both the good and poor freezable crossbred bulls at different hours of incubation.

Analysis of variance of the different CASA parameters revealed that there was no significant difference between the experimental groups at different hours of incubation except curvilinear velocity and amplitude of lateral head displacement at 0 hours, which was showing highly significant difference $(\mathrm{p}<0.01)$.

The post insemination conception rate in various semen additives showed that the glutathione treated extender has registered highest no. of pregnancies to the tune of $72.00 \%$ and $59.00 \%$ respectively in good and poor freezable crossbred bulls out of 51 and 47 artificial inseminations. Similarly the overall pregnancy rate was $60.00 \%$ and $56.00 \%$ in cysteine added frozen semen, out of 41 and 44 artificial breeding. The lowest value of $51.00 \%$ and $48.00 \%$ was observed in control group where no additives were used in a routine frozen semen extender (EYTG). The glutathione treated group recorded a highest conception rate of $66.00 \%$ and it was lowest $(49.00 \%)$ for control untreated group. Comparison of conception rate between experimental groups revealed no significant difference by chi-square analysis. Similarly the pregnancy percentages registered by each bull did not differ significantly and the overall value between applications also was not significant, but glutathione had higher percentage of pregnancy. 


\section{CONCLUSION}

The present investigation was undertaken with a view to assess the routine seminal traits and motility by computer assisted semen analysis in relationship to conception rate from both good and poor freezable crossbred Jersey bulls semen, consequent to addition of cysteine and glutathione.

The salient findings from the investigation were highlighted as below

The routine semen analysis revealed significant difference in various seminal attributes between good and poor freezable bull semen.

Motility, livability, total sperm abnormality, loss of acrosomal integrity, percent of HOS positive sperm and cervical mucus penetration test $(\mathrm{mm} /$ hour) were significantly different in various experimental group exhibiting significantly higher values in glutathione added extender (Gr III) with respect to various seminal attributes.

Percentages of DNA fragmentation recorded a highly significant difference between Gr I (control) and Gr III (glutathione).

Computer assisted sperm analysis indicated no significant difference between good and poor freezable crossbred bull's semen with respect to forward progressive motility at different hours of incubation from 0 to 4 hours.

Comparison of addition of glutathione and cysteine revealed a non significant higher value with respect to progressive forward motility and total motility following 2 hours and 4 hours of incubation indicating better mobility in additive groups compared to control.

The various mobility patterns with respect to VCL, VSL, VAP, LIN and BCF were demonstrated to be higher in group III and group II in comparison to the control group consequent to 2 hours of incubation and above.

The field fertility trials revealed highest pregnancy rate in glutathione added group semen followed by cysteine and control in both good and poor freezable semen indicating beneficial effect of the additives in long term storage of semen.

From the above observation, the use of glutathione and cysteine hcl as semen additives may be recommended for overall augmentation of pregnancy in cows. However, authentication of these additives in semen may be validated by experimenting in a larger population. 


\section{BIBLIOGRAPHY}

Abdel Raouf, M. (1965). Sexual behaviour and semen picture of bulls of the Swedish Red and White breed between the ages of 9 and 15 months. Nord. Vet. Med., 17:818-822 (Anim. Breed. Abstr., 34:247).

Agarwal, A., Prabakaran, S.A. and. Said, T. M. (2005). Prevention of Oxidative Stress Injury to Sperm. J. Androl., 26(6):654-660.

Ahmadi, A.N.S.C. (1999). Fertilizing ability of DNA-damaged spermatozoa. J. Exp. Zool., 284(6):696-704.

Aitken, J. (1995). Mechanisms of prevention of lipid peroxidation in human spermatozoa. In Human acrosome reaction. Eds. P.Fenichel and J. Parinaud. PP 339-353.

Aitken, R.I., Clarkson, J.S. and Fishel, S. (1989). Generation of reactive oxygen species, lipid peroxidation and human sperm function. Biol Reprod., 40: 183-197.

Aitken, R. J. and Clarkson, J. S. (1987). Cellular basis of defective sperm function and its association with the genesis of reactive oxygen species by human spermatozoa. J.Reprod. Fertil., 81: 459-69.

Aitken, R. J., Sutton, M., Warner, P. and Richardson, D.W. (1985). Relationship between the movement characteristics of human spermatozoa and their ability to penetrate cervical mucus and zona free hamster oocytes. J. Reprod. Fertil., 73: 441-449.

Aitkin, R. J. (1990). Motility parameters and fertility. In: Gagnon, C. (Ed.), Control of Sperm Motility; Biological and Clinical Aspects. CRS Press, Boca Raton, pp. 285-302.

Alexei, S., Wilfredo, H., Rómulo, S., Teodosio, H., Néstor, S. and Raúl, S. (2005). Effects on the quality of frozen-thawed Alpaca (Lama pacos) semen using two different cryoprotectants and extenders. Asian J. Androl., 7 (3):303-309.

Almquist, J.O. and Cunningham, D.C. (1967). Reproductive capacity of beef bulls. 1. Post puberal changes in semen production of different ejaculations. J. Anim. Sci., 26:174181.

Alvarez, J.G., Touchstone, J.O., Blasco, L. and Storey, B.T. (1987). Spontaneous lipid peroxidation and production of hydrogen peroxide and superoxide in human 
spermatozoa: superoxide dimutase as a major enzyme protectant against oxygen toxicity. J. Androl., 23:338-348.

Alvarez, J.G. and Storey, B.T. (1989) Role of glutathione peroxides in protecting mammalian spermatozoa from loss of motility caused by spontaneous lipid peroxidation. Gamete Res., 23: 77-90.

Alvarez, J.G. and Storey, B.T. (1982). Spontaneous lipid peroxidation in rabbit epididymal spermatozoa: its effect on sperm motility. Biol. Reprod., 27: 1102-1108.

Alvarez, J.G., Touchstone, I.C., Blasco, L. and Storey, BT. (1987). Spontaneous lipid peroxidation and production of hydrogen peroxide and superoxide in human spermatozoa. J. Androl., 8:338-48.

Amit, A., Bergman, A., Yedwab, G., David, M.P., Homonnai T.Z. and Paz, G. (1982). Penetration of human ejaculated spermatozoa into human and bovine cervical mucus. II. Pattern and velocity of penetration .Int. J. Frtil., 27:160-165.

Amelar, R.D., Dubin, L. and Schoenfeld, C. (1980). Sperm motility. Fertil Steril., 34(3):197215

Anil kumar, R. and Devanathan, T.G. (1995). In-vitro penetration of bovine cervical mucus by frozen bull spermatozoa. Indian J.Anim.Reprod. 16(2):109-110.

Anzar, M., Hassan, M.M., Graham, E.F., Deyo, R.C.M. and Singh, G. (1991). Efficacy of the Hamilton-Thorn motility analyzer (HTM-2030) for the evaluation of bovine semen. Theriogenology, 36:307-17.

Arruda, R.P., Souza, N.L., Marques, A., Celeghini, E.C.C., Gobesso, A.A.O., Meirelles, F.V., Binelli,M. and Blasques, FJH.(2002). Evaluation of techiniques using CFDA/PI, H258/FITC-PSA and Trypan Blue/Giemsa for assessment of the viability and acrosomal Integrity of cryopreserved equine spermatozoa. Theriogenology, 57(1):477 (Abstract).

Auger, J., Ronot, X. and Dadoune, J.P. (1989). Human sperm mitochondrial function related to motility: A flow and image cytometric assessment. J. Androl., 10: 439-48.

Austin, C.R. and Bishop, M.W.H. (1958). Role of the rhodent acrosome and perforatorium in fertilization. Proc. R. Soc., 149: 241-248. 
Bakst, M. R. and Cecil, H. C. (1992). Effect of bovine serum albumin on motility and fecundity of turkey spermatozoa before and after storage. J.Reprod. and Ferti., 94:287-293.

Bannai, S. (1984). Transport of cystine and cysteine in mammalian cells. Biochem. Biophys. Acta., 779:289-306.

Barik, A.K., Mohanty, B.N., Ray, S.K.H. and Mohanty, D.N. (1987).Fructose and citric acid concentration and its relation with seminal attributes in bull semen. Indian J.Anim.Reprod., 8(2):84:87.

Bartlett, F.D. (Jr) and Van Demark. (1961). Effect of tris amino methane on spermatozoa livability. J. Anim. Sci., 20:965 (Abstract).

Bartoov, B., Bar-Sagie, D. and Mayevsky, A. (1980). The effect of pH on ram sperm collective motility driven by mitochondrial respiration. Intl. J. Androl., 3:602-612.

Baumber, J., Ball, B. A., Gravance C.G., Victor, M. and Mina C. G. D. (2000). The Effect of Reactive Oxygen Species on Equine Sperm Motility, Viability, Acrosomal Integrity, Mitochondrial Membrane Potential, and Membrane Lipid Peroxidation. J.Androl., 21:895-902.

Bedi, K.S., Biswas, R.K. and Durgwekar, Y.G. (1984). Fructolysis in semen and its relation to breeding performance in buffalo bull. Indian J. Anim.Reprod., 4(2): 60-63.

Belorkar, P.M., Dhami, A.J., Derashri, H.J. and Kodagali, S.B. (1990) Studies on seminal mensurational characteristics of spermatozoa and their interrelationship with freezability and fertility in cross bred bull. PVK Res. J. 14: 165-173 (Anim. Bred. Abstr. 61:769).

Berndtson, W. E., Olar, T. and Pickett, B. W. (1981). Correlation between post-thaw motility and acrosomal integrity of bovine sperm. J. Dairy Sci., 64:346-349.

Bhardwaj, A., Verma, A., Majumdar, S. and Khanduja, KL. (2000). Status of vitamin E and reduced glutathione in semen of oligozoospermic and azoospermic patients.Asian $J$. Androl., 2(3):225-8.

Bhaskaran, R. and Dubey, B.M. (2005) Effect of gonadotropin releasing hormone to augment semen production in Jersey bulls. Indian J. Reprod., 26: 14-16. 
Bhatia, H., Sidhu, S.S. and Singh, P. (2007). Evaluation of dairy bull semen Vis-a- Vis sperm migration capacity and field fertility trials. XXIII Annual Convention of ISSAR and national symposium .Bhubaneswar. Pp- 233-234.

Bhoite, U.Y., Satar D.A. and Ulmet B.R. (2008). Studies on semen quality of cross bred bulls. Ind.Vet.J., 85:395-397.

Bhosrekar, M.R. (1990). Semen production and artificial inseminaton. BAIF Publication, Pune, India.

Bhostrekar, M.R., Purohit, J.R. and Mangur Khar, B.R. (1990). Studies on the effect of additives to semen diluent. Indian J.Anim.Reprod., 11:2:85-88.

Bilodeau, J.F., Blanchette, S., Gagnon, C. and Sirard, M.A. (2001). Thiols prevents $\mathrm{H}_{2} \mathrm{O}_{2}$ mediated loss of sperm motility in cryopreserved bull semen. Theriogenology, 56(2):275-86.

Bishop, M.W.H., Campbell, R.C., Hancock, J.L., and Watson, A. (1954). Semen characteristics and fertility in bulls. J. Agric. Sci., 44:227-248.

Blom, E. (1950a). The evaluation of bull's semen with special reference to its use in artificial insemination. Copenhagen: A/s cart. Fr. Moutensen. pp.223 (Anim. Breed. Abstr., 19:648).

Blom, E. (1950b). En hurtig-farvningsmethode till adskillelse of levende og dq de spermier ved hiael $\mathrm{p}$ af Eosin-Nigrosin. Nord. Vet.-Med., 2:58.

Bollwein, H., Fuchs, I. and Koess, C. (2008). Inter-relationship between plasma membrane integrity, mitochondrial membrane potential and DNA fragmentation in cryopreserved bovine spermatozoa. Reprod. Domest. Anim. Sci., 43(2):189-95.

Bonnadonna, T. and Kann, I. (1955). Relationship between bovine fertility and the number of sperm presumed living per cubic $\mathrm{cm}$ used in artificial insemination. Vet. Ital. 6:333-350 (Anim. Breed. Abstr., 23:1694).

Brahmkshtri, B.P., Edwin, M.J., John, M.C., Nainar, A.M. and Krishnan, A.R. (1999). Relative efficacy of conventional sperm parameter and sperm penetration bioassay to assess bull fertility in vitro. Anim. Reprod. Sci., 54:159-168. 
Branton, C., James, C.B., Patrick, T.E. and Newson, M.H. (1951). The relationship between certain semen quality tests and fertility and interrelationship of these tests. J. Dairy. Sci., 34:310-316.

Bredderman, P.J. and Foote, R.H. (1969) Volume of stressed bull spermatozoa and protoplasmic droplets and relationship of cell size to motility and fertility. J. Anim. Sci., 28: 496-501.

Bruno, L. and Jacques, T. (1996). Lectins binding on human sperm surface increase membrane permeability and stimulate acrosomal exocytosis Mole.Human Reprod., 2(9): 651-658.

Buckett, W.M., Luckas, M.J., Aird, I.A., Farquharson, R.G., Kingsland, C.R. and Lewis-Jones, D.I. (1997). The hypoosmotic swelling test in recurrent miscarriage. Fertil. Steril., 68: 506509.

Budworth, P.R., Amann, R.P. and Hammerstedt, R.H. (1987). A microcomputer-photographic method for evaluation of motility and velocity of bull sperm. J. Dairy Sci., 70:1927-36.

Buege, J.A. and Aust, S.D. (1978). Microsomal lipid peroxidation. Methods enzymol., 52: 302-310.

Bukowinski, T. and Kucharski, J. (1989) A comparison of insemination of cows with semen preserved in straws or in pellets. Pizeglad Hodowlany, 57:15-16 (Anim. Breed. Abstr., 58:4209)

Celeghini, E.C.C., Arruda, R.P., Albuquerque, R., Silva, F.H.A., Faria, D.E., Andrade, A.F.C., Nascimento, J. and Raphael, C.F. (2007). Utilization of fluorescent probe association for simultaneous assessment of plasmatic, acrosomal, and mitochondrial membranes of rooster spermatozoa. Braz. J. Poul.Sci., 9(3):143 - 149.

Chatterjee, S., de Lamirande, E. and Gagnon, C. (2001). Cryopreservation alters membrane sulfhydryl status of bull spermatozoa: protection by oxidized glutathione. Mol. Reprod. Dev., 60(4):498-506.

Chauhan, F.S., Matharoo, J.S., Takkar, O.P. and Singh, M. (1983). Semen characteristics, deep freezing of semen and reproductive performance of crossbred cattle. Indian $J$. Dairy Sci., 36: 96-100. 
Chauhan, F.S., Mathew, J.S., Takkav, D.D. and Singh, M. (1983). Semen characterstics, deep freezing of semen and reproductive performance of CB cattle. Ind.J.Dairy Sci., 36:96100.

Chen, Y., Li, J., Simkin, M.E., Yang, X. and Foote, R.H. (1989). Fertility of fresh and frozen rabbit semen inseminated at different times is indicative of capacitation time. Biol. Reprod., 41:848-853.

Chenoweth, P.J. (1980). Bull fertility. Modern Vet. Prac., 61:987-991.

Clarke, GN., Garrett, C. and Baker, GHW. (1998). Quantitative sperm mucus penetration: modified formulae for calculating penetration efficiency. Hum. Reprod., 13: 12551259.

Coetzee, K., Kruge, T.F., Menkveld, R., Lombard, C.T. and Swanson, R.J. (1989) Hypo- osmotic swelling test in the prediction of male fertility. Arch. Androl., 23: 619-628.

Colchen - Bourlaoud, M.A. and Thibier, M. (1973). Information on the sexual function of the young sire. Elevage et Insemination, 136:3-37 (Anim. Breed. Abstr. 42:1419).

Comporti M. (1989). Three models of free radical-induced cell injury. Chemico- Biol Interact., 72:1-56.

Cotran, R.S., Kumar, V. and Robbin, S.L. (1989). Pathologic Basis of Diseases, 4th edn. WB.Saunders Co., Philadelphia, PA, pp.9-16.

Correa, J.R., Heersche, Jr, G. and Zavos, P.M. (1997). Sperm membrane functional integrity and response to frozen thawed bovine spermatozoa during the hypoosmotic swelling test incubation at varying temperatures. Theriogenology, 47: 715-721.

Cross, N.L, Meizel, S. (1989). Methods for evaluating the acrosomal status of mammalian sperm. Biol. Reprod., 41: 635-641.

Cross, N.L and Watson, S.K. (1994). Assessing acrosomal status of bovine sperm using fluoresceinated lectins. Theriogenology, 42:89-99.

Cross,N.L. and Overstreet, J.W. (1987). Glycoconjugate of the human sperm surface: distribution and alterations that accompany capacitation in vitro. Gamete Res., 16:2335 . 
Cross, N.I., Morales, P., Fukuda, M. and, Behboodi, E. (1988). Determining acrosomal status of the Cynomolgus monkey (Macaca fascicularis) sperm by fluorescence microscopy. American J. Primat., 17 (2): 157 - 163

Curtis, G.G., Duane, L.G., Marion, G.M. and Trish, B. (2000). Fluorescent probes and flow cytometry to assess rat sperm integrity and mitochondrial function. Reprod.Toxico., 15(1): 5-10.

Dabas,V.P.S. and Maurya, S.N. (1988). A field method for collection of bovine cervical mucus for microbiological studies. Indian J. Anim. Reprod., 9: 138-139.

Das, H.N. (1994) Studies on fertilizing ability of spermatozoa using super ovulated donors and different in vitro techniques in Cross Bred cattle. M.V.Sc. Thesis, IVRI, Izatnagar, U.P., INDIA.

David, M.P., Amit, A., Bergman, A., Yedwab, G., Paz, G.F. and Homonnai, Z.T. (1979) Sperm penetration in vitro: correlations between parameters of sperm quality and the penetration capacity. Fertil. Steril., 32: 676-680.

Davis, I.S., Bar Hon, R.W. and Foote, R.H. (1963). Livability of bovine spermatozoa at $5^{0} \mathrm{C}$ in Tris buffered and citrate buffered yolk glycerol extenders. J. Dairy. Sci., 46:57.

De Leeuw, AM., Den Daas, JH. and Woelders, H. (1991). The fix vital stain method. Simultaneous determination of viability and acrosomal status of bovine spermatozoa. J. Androl., 12: 112-118.

Dhami, A.J., Sahni, K.L. and Gresh Mohan. (1991). Effect of pre freeze holding time (at $5^{\circ}$ C) and thawing rates on post thaw motility and thermosesistance of bubaline and taurine spermatozoa. Indian J.Anim.Reprod., 12(2):129-134.

Dhingra, S.O., Hukeri, V.B., Deshpande, B.R., Shah, G.V. and Sheth, A.R. (1984). Revival rate of frozen buffalo bull spermatozoa with addition of epididymal extract (PMSF). Indian $J$. Anim.Reprod., 4(2):57-59.

Dojcseva, M., Sojanov, T. and Tuncsev, T. (1979). Comparative study on fructose and citric acid levels of bull semen in various seasons. Magyar Allatorvosok Lapja, 34:243-245.

Donoghue, A.M., Holsberger, D.R., Evenson, D.P. and Froman, D.P. (1998). Semen donor selection by in vitro sperm mobility increases fertility and semen storage in the Turkey Hen. J. Androl., 19(3):295-301. 
Drevius, L.O. and Eriksson, H. (1966) Osmotic swelling of mammalian spermatozoa. Expt .cell Res., 42 : 136-156.

Edwin, M.J., Rodricks, I.M. and Rathna Sabapathy, V. (1975). Comparative merit of tris, sodium citrate and sodium bicarbonate glucose as extenders of bovine semen. Indian Vet. J., 52:345.

Ellington, J., Scarlett, J., Meyers-Wallen, V., Mohammed, H.O. and Surman, V. (1993). Computer assisted sperm analysis of canine spermatozoa motility measurements. Theriogenology, 40:725-33.

ESHRE (European Society of Human Reproduction and Embryology) Andrology Special Interest Group. Consensus workshop on advanced diagnostic andrology techniques. Hum Reprod. 1996; 11:1463-1479.

ESHRE (European Society of Human Reproduction and Embryology) Andrology Special Interest Group. Guidelines on the application of CASA technology in the analysis of spermatozoa. Hum Reprod. 1998; 13:142-145

Erb, R.E., Ehlers, M.H. and Grassner, F.X. (1956). Metabolism of bull semen and fructolysis relationship with sperm concentration and fertility. J. Dairy Sci., 39:326-331.

Eskiocak, S., Gozen, A.S., Yapar, S.B., Tavas F., Kilic, A.S. and Eskiocak, M. (2005). Glutathione and free sulphydryl content of seminal plasma in healthy medical students during and after exam stress. Human Reprod., 20 (9): 2595-2600.

Evenson, D.P. and Wixon, R. (2006). Clinical aspects of sperm DNA fragmentation detection and male infertility. Theriogenology, 65: 979-991.

Evenson, D.P., Darzynkiewicz, Z. and Melamed, MR. (1982). Simultaneous measurement by flow cytometry of sperm cell viability and mitochondrial membrane potential related to cell motility. J. Histochem. Cytochem., 30: 279-80.

Farlin, M.E., Jasko, D.J., Graham, J.K., and Squires, E.L. (1992). Assesment of Pisum sativum agglutinin in identifying acrosomal damage in stallion spermatozoa. Mol. Reprod.Dev.,32: $23-27$.

Farrell, P.B., Foote, R.H., Simkin, M.E., Clegg, E.D. and Wall, R.J. (1993). Relationship of semen quality, number of sperm inseminated, and fertility in rabbits. J. Androl., 14:464471. 
Foote, R.H. (2002). The history of artificial insemination: selected notes and notables. Am. Soc. Anim. Sci., 10: 1-10.

Foote, R.H., Brockett, C.C. and Kaproth, M.T. (2002). Motility and fertility of bull sperm in whole milk extender containing antioxidants. Anim Reprod Sci., 71(1-2):13-23.

Froman, D.P. and Feltmann, A.J. (1998). Sperm mobility: a quantitative trait of the domestic fowl (Gallus domesticus). Biol Reprod., 58:379-384.

Froman, D.P., Feltmann, A.J., Rhoads, M.L. and Kirby, J.D. (1999). Sperm mobility: a primary determinant of fertility in the domestic fowl. Biol Reprod., 61:400-405.

Gaddum Rosse, P., Blandau, R.J. and Lee, W.I. (1980). Sperm penetration into cervical mucus samples and storage conditions on sperm migration in-vitro. Fertil. Steril., 35: 218221.

Gadea, J., David, G., Carmen, M. S. and Romar, R. (2005b). Supplementation of the thawing media with reduced glutathione improves function and the in vitro fertilizing ability of boar spermatozoa after cryopreservation. J. Androl., 26:749-756.

Gadea, J., Francisco, G.V., Carmen, M.S., Juan C.G.N, Sebastia, N.C.N. and David, G. (2005).Cooling and freezing of boar spermatozoa: supplementation of the freezing media with reduced glutathione preserves sperm function. J. Androl., 26:396-404.

Gadea, J., Gumbao, D., Matás, C. and Romar, R. (2005). Supplementation of the thawing media with reduced glutathione improves function and the in vitro fertilizing ability of boar spermatozoa after cryopreservation. J.Androl., 6(6): 749-56.

Gadea, J., Sellés, E., Marco, MA., Coy, P., Matás, C., Romar, R. and Ruiz, S. (2004). Decrease in glutathione content in boar sperm after cryopreservation: Effect of the addition of reduced glutathione to the freezing and thawing extenders. Theriogenology, 62(3- 4):690-701.

Garner, D. L., Pinkel, D., Johnson, L.A., and Pace, M.M. (1986). Assessment of spermatozoal function using dual fluorescent staining and flow cytometric analyses. Bio.Reprod., 34:127-138.

Garner, D.L. and Johnson, L.A. (1995). Viability assessment of mammalian sperm using SYBR-14 and Propidium Iodide. Biol. Reprod.,53: 2369-2376. 
Garner, D.L., Johnson, L.A., Yue, S.T., Roth, B.L. and Haugland, R.P. (1994). Dual DNA staining assessment of bovine sperm viability. J. Androl., 15:620-629.

Garner, D.L., Thomas, C.A., Joerg, H.W., DeJarnette, J.M. and Marshall C.E.(1997). Fluorometric assessments of mitochondrial function and viability in cryopreserved bovine spermatozoa. Biol Reprod., 57: 1401-6.

Garner, D. L. and Thomas, C. A. (1999). Organelle-specific probe JC-1 identifies membrane potential differences in the mitochondrial function of bovine sperm. Mole Reprod. Develop., 53 : 222-229.

Gemeinhardt, K. (1969). Experiences in the use of bull semen stored for a long period. Dt. Tierarztl. Wschr., 76:425-427 (Anim. Breed. Abstr. 38:2473).

Graham, J.K., Kunze, E. and Hammerstedt, R.H. (1990). Analysis of sperm cell viability, acrosomal integrity, and mitochondrial function using flow cytometry. Biol Reprod., 43:55-64.

Gravance, C.G., Vishwanath, R., Pitt, C., Garner, D.L. and Casey, P.J. (1998). Effects of cryopreservation on bull sperm head morphometry. J.Androl., 19: 704-709.

Guillaume, M., Odile, S., Philippe, D. and Rachel L. (2004). Cryopreservation induces an apoptosis-like mechanism in bull sperm. Bio Reprod., 71: 28-37.

Hafez, E.S.E. (1987). Reproduction in Farm Animals. $5^{\text {th }}$ Edn. Lea and febiger, Philadelphia. Indian Edition, K.M.Verghese Company. Post Box 7119, Bombay-400031.

Hallap, T., Nagy, S., Jaakma, U., Johannisson, A. and Rodriguez-Martinez, H. (2005). Mitochondrial activity of frozen-thawed spermatozoa assessed by Mito-Tracker Deep Red 633. Theriogenology, 63:2311-2322.

Hancock, J.L. (1951). The morphology of boar spermatozoa. J. Roy. Micr. Soc., 76: 84-97.

Hancock, J. L. (1949). Evidence of an inherited seminal character associated with infertility of Friesian bulls. Vet. Rec., 61:308.

Hancock, J.L. (1952). The morphology of bull spermatozoa. J. Expt. Biol., 29: 445-453.

Haq, I., (1949). Causes of sterility in bulls in southern England. Br. Vet. J., 05:71 
Henkel, R., Hajimohammad, M., Stalf, T., Hoogendijk, C., Mehnert, C., Menkveld, R., Gips, H., Schill, WB. and Kruger, TF. (2004). Influence of deoxyribonucleic acid damage on fertilization and pregnancy. Fertil. Steril., 81(4): 965-72.

Henkel, R., Kierspel, E., Hajimohammad, M., Stalf, T., Hoogendijk, C. and Mehnert, C. (2003).

DNA fragmentation of spermatozoa and assisted reproduction technology. Reprod Biomed Online., 7:477-84.

Hermen, H.A. and Madden, E.W. (1953) The A.I. of dairy cattle. Lucas Brothers, Columbia, Missouri, USA

Hirano, Y., Shibahara, H., Obara, H., Suzuki, T., Takamizawa, S., Yamaguchi, C., Tsunoda,H. and Sato, I. (2001). Relationships between sperm motility characteristics assessed by the computer-aided sperm analysis (CASA) and fertilization rates in vitro. J. Assist. Reprod. Genet., 18(4):213-8.

Holt, C., Holt, W.V., Moore, H.D.M., Reed, H.C.B. and Curnock, R.M. (1997). Objectively measured boar sperm motility measurements correlate with the outcomes of on-farm inseminations: results of two fertility trials. J. Androl., 18(3):312- 323.

Huszar, G. and Vigue, L. (1994) Correlation between the rate of lipid peroxidation and cellular maturity as measured by creatine kinase activity in human spermatozoa. J. Androl., 15: $71-77$.

Irvine, D.S. (1996). Glutathione as a treatment for male infertility. Rev. Reprod., 1(1):6-12.

Iwasaki, A. and Gagnon, C. (1992).Formation of reactive oxygen species in spermatozoa of infertile patients. Fertil. Steril., 57 : 409-416.

Jain M.C. and Arora N. (2003). Comparative study of glutathione concentration of bovine and bubaline sperm head, mid piece and tail. Ind.Vet.J., 80:628-631.

Jasko,D.J., Little, T.V., Smith, K., Lein, D.H. and Foote, R.H. (1988). Objective analysis of stallion sperm motility. Theriogenology, 30:1159-67.

Jeulin, C., Soumah A. and Jouannet P. (1985). Morphological factors influencing the penetration of human sperm into cervical mucus in vitro. Int. J. Androl., 8: 215-223.

Jeyandran, R.S.,Van Der Ven H.H., Perez-Pelacz,M., Crabo,B.G. and Zaneved L.J.D.(1984). Development of an assay to assess the functional integrity of the human sperm 
membrane and its relation ship to other semen characteristics. J.Reprod.Fertil., 70:21928.

Joaqu,, G., David, G., Sebastia, C., Francisco, A.G.V., Luis, A.G. and Juan, C. G. (2007).

Supplementation of the dilution medium after thawing with reduced glutathione improves function and the in vitro fertilizing ability of frozen-thawed bull spermatozoa. Inter. J.Androl., 31: 40-49.

Johnson, P.E., Flipse, R.J. and Almquist, J.O. (1954). The effect of cysteine hydrochloride on the livability of bull spermatozoa in heated skim milk. J. Dairy Sci., 38:53.

Jones, R., Mann, T. and Shamns, R. (1979). Peroxidative breakdown of phocspholipids in human spermatozoa, spermicidal properties of fatty acid peroxides and protective action of seminal plasma. Fertil. Steril.,31:531-37.

Jose I. Marti', Jose A. Cebria' N-Pe' Rez, and Teresa Muiñ o-Blanco (2000). Assessment of the acrosomal status of ram spermatozoa by RCA lectin-binding and partition in an aqueous two- phase system. J. Androl., 21: 541-548.

Joshi, N., Kodwany, G., Balaiah, D., Parikh, M. and Parikh, F. (1996). The importance of computer-assisted semen analysis and sperm function testing in an IVF program. Int. J. Fertil., 41:46-52.

Joshi, S.C., Rawat, J.S. and Roy, A. (1968). Preservation of spermatozoal viability in diluents made up of amino acids with milk and/or egg yolk as base and study of biochemical and chemical characters of these diluents. Indian J. Vet., 38:243.

Jouannet P. and Feneux D.(1987). Sperm analysis. Ann. Bio.l Clin. (Paris), 45: 335-339.

Kasai, T., Ogawa, K., Mizuno, K., Nagai, S., Uchida, Y., Ohta, S., Fujie, M., Suzuki, K., Hirata, S. and Hoshi K. (2002). Relationship between sperm mitochondrial membrane potential, sperm motility, and fertility potential. Asian J Androl., 4(2):97-103.

Katz, D.F., Overstreet, J.W. and Drolonis, E.Z. (1989). Factors regulating mammalian sperm migration through the female reproductive tract and oocyte vestments. Gamete Res., 22:443-469.

King, L.M., Holsberger, D.R. and Donoghue, A.M. (2000). Correlation of CASA Velocity and linearity measurements with sperm mobility phenotype in turkeys. J. Androl., 21:65-71. 
Kkjoestad, H., Ropstad, E. and Berg, A.K. (1993). Evaluation of spermatological parameters used to predict the fertility of frozen semen. Acta. Vet. Scand., 34:199-303.

Konstandoglo, A.G. (1989). Cytogenetic monitoring of cattle on Moldovia. Referativnyi Zhumal, 6:315 (Anim. Breed. Abstr., 59:3163).

Kremer, J.and Kroeks M.(1975). Modification of the in vitro spermatozoal penetration test by means of the sperm penetration meter. Acta Eur. Fertil., 6:377-380.

Krogenaes, A., Anderson, B.K., Hafne, A. and Engeland, E. (1994). Membrane alterations in bull spermatozoa after freezing and thawing and after in vitro fertilization. Acta Vet. Scand.,35:17-26.

Kumaresan, A., Ansari, M.R., Abhishek, G. and Meena, K. (2006). Effect of oviductal proteins on sperm functions and lipid peroxidation levels during cryopreservation in buffaloes. Anim Reprod Sci., 93(3-4):246-57.

Lardy, H.A. and Phillips, P.H. (1941). The effect of certain inhibitions and activations in sperm metabolism. J. Biol. Chem., 138: 195-202.

Larsen, L., Scheike, T. and Jensen, T.K. (2000). Computer-assisted semen analysis parameters as predictors for fertility of men from the general population. Hum. Reprod.,15:15621567.

Lasley, J. F., Easley, G. T. and McKenzie, F. F. (1942). A staining method for the differentiation of live and dead spermatozoa. I. Applicability to the staining of ram spermatozoa. Anat. Rec. 82:167.

Lasley, J.F. (1951). Spermatozoan motility as a measure of semen quality. J. Anim. Sci., 10:211218.

Lasley, J.F. and Bogart, R. (1943). Some factors influencing reproductive efficiency of range cattle under artificial and natural breeding conditions. Res. Bull. Mo. Agric. Exp. Sta. No. 375 (cited by Bishop et al., 1954).

Lee, W.I., Gaddum-Rosse P. and Bladau R.J. (1981). Sperm penetration into cervical mucus invitro.III. Effect of freezing on estrous bovine cervical mucus. Fertil. Steril.,36:209. 
Leidl, W., Kato, H., Hollerrieder, J. and Braun, J. (1993). Analysis of sperm motility by computer assisted methods, with special consideration of in vitro fertilization. Mol Reprod Dev, 36:222-228.

Liu, D.Y., Clarke, G.N. and Baker, H.W. (1991). Relationship between sperm motility assessed with the Hamilton-Thorn motility analyzer and fertilization rates in vitro. J.Androl., 12:231-239.

Lundgren, B. (1980). Influence of long term storage on fertility of deep frozen bull semen. Nordisk Vet., 32:427-432.

Macleod, J. (1943). The role of oxygen in metabolism and motility of human spermatozoa. Am. J.Physiol., 138: 512-518.

MacLeon, I.C. and Irvine, D.S. (1995). The Predictive Value of Computer-assisted Semen Analysis in the Context of a Donor Insemination Programme. Human Reprod., 10:580586.

Madden, F.W., Herman, H.A. and Berousekk, E.R. (1947) Res. Bull. Mo. Agric. Exp. Sta. No. 407 (cited by Bishop et al., 1954).

Madeja, Z., Waroczyk, M., Strabel, T. and Lechniak. D. (2003). Use of the hypo osmotic swelling test for evaluating bull and boar semen quality. Theriogenology, 59(12): 105-1156.

Magargee, SF., Kunze, E. and Hammersted, RH. (1988). Changes in Lectin-Binding Features of Ram Sperm Surfaces Associated with Epididymal Maturation and Ejaculation. Biol. Reprod., 38:667-685.

Mandal, D.K., Tyagi S. and Mathur A.K. (2005). Semen production performance of sahiwal bulls. Ind. J. anim. Sci.., 75(1):17-19.

Mandal, D.K., Tyagi, S., Kumar, M. and Mathur, A.K. (2007). Cyto - morphological abnormalities and cell membrane integrity of frozen thawed spermatozoa of Frieswal bulls and their relationship with fertility. Indian J.Anim.Reprod., 28(2):30-33.

Mandal,D.K., Nagpaul, P.K. and Gupta, A.K. (2003) Motion characteristics of Murrah buffalo bull spermatozoa in various seasons and its relationship with functional integrity of the plasmallema. Theriogenology, 60: 349-358 
Mann, T. and Lutwak-Mann, C. (1981). Male reproductive function and semen Springer Verlag, Berlin, Heidelberg, New Work, USA.

Marchetti, C., Obert, G., Deffosez, A., Formstecher, P. and Marchetti, P.(2002). Study of mitochondrial membrane potential, reactive oxygen species, DNA fragmentation and cell viability by flow cytometry in human sperm. Hum. Reprod., 17:1257- 1265 .

Marshburn, P.B., Mclntire, D., Carr, B.R., and Byrd,W. (1992). Spermatozoal characteristics from fresh and frozen donor semen with their correlation with fertility out come after intrauterine insemination .Fertil.Steril., 58:179-186.

Mathew, A. (1988). Problems involved in freezing of cross bred bull semen. A.I. News, 111:2023.

Mathur, A.C., Bisht, G.S., Mohan, G. and Sahni, K.L. (1991) Development of some rapid "Test freezing methods" for predicting the freezability of bovine semen. Indian J. Anim. Reprod., 12 : 6-12.

Maule, J.P. (1962). The semen of animals and artificial insemination. $1^{\text {st }}$ Edn. CAB Farnham, Royal books, England.

Maxwell, W.M.C. and Stojanov, T. (1996). Liquid storage of Ram semen in the absence or presence of some antioxidants. Reprod. Fertil. Dev., 8: 1013-20.

Mayer, D. T., Squiers, C. D., Bogart, R. and Oloufa, M. M.. (1951). The technique for characterizing mammalian spermatozoa as dead or living by differential staining.J. Anim. Sci., 10:226.

Meister, A. and Tate, S.S. (1975). Glutathione and related gamma-glutamyl compounds: Biosynthesis and utilization. Annu.Rev. Biochem.,45: 559-604.

Moharatha, R.M. (2001). Studies on freezability and conception rate in breeding bulls. M.V.Sc. Thesis, OUAT, Bhubaneswar, Orissa, India.

Mohanty, D.N. (1981) Semen characteristics and their correlation with testicular and body measurements in Holstein Friesian bulls. M.V.Sc. Thesis submitted to PAU, Ludhiana, India.

Moore, H.D.M. and Akhondi, M.A. (1996). Fertilizing capacity of rat spermatozoa is correlated with decline in straight-line velocity measured by continuous computer-aided sperm 
analysis: epididymal rat spermatozoa from the proximal cauda have a greater fertilizing capacity in vitro than those from the distal cauda or vas deferens. J. Androl., 17:5060.

Mortimer, D. (1994). Practical Laboratory Andrology. New York: Oxford University Press.

Mortimer, S.T. (1997). A critical review of the physiological importance and analysis of sperm movement in mammals. Human Reprod Update, 3:403 - 439

Mostari, M.P., Hasanat, M.S., Azmal, S.A., Monira, K.N. and Khawn, H. (2005). Effect of seasonal variation on semen quality and herd fertility. Pakistan Journal of Biological Sciences, 8: 581-585 (Dairy. Sci. Abstr. 67: 10).

Moustafa, A.R. and Meszaros, I. (1980). Interrelationship between the total protein content of bovine seminal plasma and behaviour of the spermatozoa after freezing and thawing. Acta. Vet. Acad. Sci., Hung. 28:403-408.

Munsi, M.N., Bhuiyan, M.M.U., Majumder, S. and and Alam, MGS. (2007). Effects of exogenous glutathione on the quality of chilled bull semen. Reprod. Dom. Anim., 42: $358-362$.

Mustafa N. Bucak and Necmettin Tekin. (2007). Protective effect of taurine, glutathione and trehalose on the liquid storage of ram semen Theriogenology, 73(1-3): 103-108.

Nair, A.K. (1997). Studies on cryo preservation of Cross Bred bull semen in relation to osmolarity, biochemical indices and in vitro fertility tests. M.V.Sc. Thesis, IVRI, Izatnagar, U.P., INDIA.

Nair, M., Kalatharan, S.R. and Rajasekaran.J. (1999). Effect of cryopreservation on the viability and membrane integrity of canine spermatozoa. Ind. J. Anim.Reprd., 20(2):142-145.

Nehring, H. (1988). Evaluation of acrosome integrity during semen storage. Medizin, 37:109115 (Anim. Breed. Abstr., 57:3064).

Nishiyama, H., Ogawa, K., Nakamishi, Y. (1968b). The inter relation between semen characters of bull. Bull. Fac. Agric. Kagoshima Univ., 18:165-167. (Anim. Breed. Abstr., 36:3601).

Nishiyama, H., Ogawa, K., Nakamishi, Y. and Oyamad, H. (1968a) .Seasonal variation in semen quality of the Holstein bulls. Bull. Fac. Agric. Kagoshima Univ., 98:157-164. (Anim. Breed. Abstr., 36:3602). 
O’Connor, M.T., Amann, R.P. and Saacke, R.G. (1981). Comparisons of computer evaluations of spermatozoal motility with standard laboratory tests and their use for predicting fertility. J.Anim. Sci., 53:1368-1376.

Olds - Clarke, P. (1996). How does poor motility alter sperm fertility ability? J. Androl., 17:183186

Panday A.K. and Gupta H.P.(2004). Evaluation of various dilutors by acrosome reaction test. Ind. J. Anim. Res., 38(2):112-116.

Pant, H.C., Mittal, A.K., Patel, S.H, Shukla, H.R., Kasi Raj R. and Prabharan J.H. (2002). The hypo-osmotic swelling test: an assay of cell membrane integrity and quality of frozen thawed buffalo semen. Ind. J. Anim.Reprd., 28(1): 8-11.

Parkinson, J.J. and Whit field, C.H. (1987). Optimization of freezing conditions for bovine spermatozoa. Theriogenology, 27:781-797.

Parks, J.E. and Graham, J.K. (1992). Effect of cryopreservation procedures on sperm membranes. Theriogenology, 38:209-222.

Patil S.K., Honnappagol S.S., Arora V.K. and Tandle M.K. (2008). Lecithin and tris based new diluents on fertility of bull semen. Indian J. Anim.Reprod., 85:219-220.

Pattabiraman, S.R., Venkaswamy, V. and Krishnamoorthy. T. (1967). Effects of pH and viscosity of estrous mucus of cows on the physiology of spermatozoa. Indian Vet. J., 44:767-772.

Paulenz, H., Grevle, I.S., Tverdal, A., Hofmo, P.P. and Berg, K.A. (1995). Precision of the coulter(R) counter for routine assessment of boar sperm concentration in comparison with the Hemocytometer and Spectrophotometer. Reprod. Demos. anim., 30: 107-111.

Pena F.J., Johannisson A., Wallgren M. and Martiner H. (2004). Effect of hyaluron supplementation on boar sperm motility and membrane lipid architecture status after cryopreservation. Theriogenology, 61:63-70.

Pena, F. J., Johannisson, A., Wallgren, M. and Rodriguez, M. H. (2003). Antioxidant supplementation in vitro improves boar sperm motility and mitochondrial membrane potential after cryopreservation of different fractions of the ejaculate. Anim Reprod. Sci., 78:85-98. 
Perez, Y. and Perez, F. (1954). The role of enzymes in sterility, Hyaluronidase and Vitamin C. An. Inst. Invest., Vet., 6:145 (Anim. Breed. Abstr., 24:1111).

Pintado, B., De La Fuent, J. and Roldan, E.R.S. (2000). Permeability of boar and bull spermatozoa to the nucleic acid stains Propidium iodide or Hoechst 33258 or to eosin: accuracy in the assessment of cell viability. J.Reprod.Fertil., 118:145-152.

Polge, C. and Rowson, L.E.A. (1952). Fertility capacity after freezing at $-79^{0}$ C. Nature, $\quad$ 169:626.

Porwel, M.L., Saxena, H.K. and Karandikar, G.W.(1977) Effects of seasons on seminal attributes of Red Dane bulls. Indian Vet. J., 54: 163-166.

Raina, C.M. and Dhami, A.J. (2004). Physical attributes intact acrosome, HOS test and freezability of semen of Gir and Jaffarabady bulls.Ind.Vet.J. 81:406-410.

Rao, A.V.N., Harnath, G.B. and Somasekharam, G. (1993). Revivability and acrosome integrity of buffalo spermatozoa deep frozen in straws using different equilibration and thaw rates. Indian J. Anim.Reprod., 14(1):44-47.

Rao, B.V., Chetty, A.V., Ramachandraiah, A.V. and Sreeraman, P.K. (1991). Preservation of native boar semen. Indian J. Anim.Reprod., 12(2):148-150.

Rao, K., Rao, A.S., Naidu, K.S.,Naidu, K.V. and Rao, M.M. (2002). Post thaw evaluation and fertility of bovine semen diluted in biociphos plus and tris extender. Indian J.Anim.Reprod., 23(1): 29-31.

Rao, A.R. and Rao, T.L.N. (1979) Change in the morphology of bovine spermatozoa during preservation and storage. Indian Vet. J., 56:294-296.

Rao, K.B. and Rao, A.R. (1991). Evaluation of cross bred bulls for breeding soundness. Indian J. Anim. Reprod., 12:111-113.

Reddenz (1933) Fructolysis in the semen of animals and artificial insemination 43 (Cited by Maule, J.P. 1962. C.A.B., England).

Reers, M., Smith, T.W., and Chen, L.B. (1991). J-aggregate formation of a carbocyanine as a quantitative fluorescent indicator of membrane potential. Biochemistry, 30:4480-4486.

Reichmann, J., Insler, V. and Serr, D.V. (1973). A modified in-vitro spermatozoal penetration test. Int. J. Fertil., 18: 241-245. 
Revel R.G. and Mrode R.A. (1994). An osmotic resistance test for bovine semen.Anim. Reprod. Sci., 36:77-86.

Roberts, S.J. (1971) Infertility in male animals. Veterinary obstetrics and genital diseases. Published by the author, Ithaca, N.Y.

Roca, J., Rodriguez, M.J., Gil, M.A., Carvajal, G., Garia, E.M., Cuello, C., Vazquoz, J.M. and Martinez, E.A. (2005) Survival and in vitro fertility of boar spermatozoa frozen in the presence of super oxide Dismutase / Catalase. J. Androl., 26: 35-39.

Rollinson, D.H.W. (1951). Br. Vet. J., 107:4, 6, 11, 203, 258(Cited by Venkataswamy, V. and Vedanayagam, A.R. 1962. Biometrics of spermatozoa of cattle and buffaloes. Indian Vet. J., 39: 287-291).

Rota, A., Penzo, N., Vencenti, L. and Mantovani, R. (2005). Hypoosmotic swelling (HOS) as a screening assay for testing in vitro fertility of bovine spermatozoa. Theriogenology, 53:1415-20.

Saacke, R.G. (1970). Morphology of sperm and its relationship to fertility. Proc. $3^{\text {rd }}$ Tech. Conf. A.I. and Reprod. N.A.A.B., pp. 17.

Saacke, R.G., Dejarnette, J.M., Bame, J.H., Karabinus, D.S. and Whitman, S.S. (1998). Can spermatozoa with abnormal heads gain access to the ovum in artificially inseminated super- and single-ovulating cattle? Theriogenology, 50: 117-128.

Sagara J., Miura K. and Bannai S.J. (1993). Cystine uptake and glutathione level in fetal brain cells in primary culture and in suspension. Neurochem., 61:1667-1671.

Sagdeo, L.R., Chitnis, A.B. and Kaikini, A.S. (1992) Semen freezability in relation to single or multiple exotic genetic components in CB bulls. Indian J. Anim. Reprod., 13: 131-133.

Sagdeo, L.R., Chitnis, A.B., Deshmukh, S.N. and Kaikini, A.S. (1990) Studies on semen freezability of pure Jersey with varying levels of exotic inheritance. Indian J. Anim. Reprod., 11: 79-84.

Sagdeo, L.R., Chitnis, A.B., Deshmukh, S.N. and Kaikini, A.S. (1980). Seasonal variations in relation to freezeablity of semen of Jersey and CB bulls with varying levels of exotic inheritance. Indian J. Anim. Reprod., 12: 117-121. 
Salamon, S. and Maxwell, W.M.C. (1995). Frozen storage of ram semen II. Causes of low fertility after cervical insemination and methods of improvement. Anim. Reprod. Sci., 38:1-36.

Salisbury, G.W. and Bratton, R.W. (1948) Fertility level of bull semen diluted at 1:400 with and without sulfanilamide. J. Dairy. Sci., 31:817.

Salisbury, G.W., Fuller, H.K. and Willett, E.L. (1942) Preservation of bovine spermatozoa in yolk citrate diluents and field results from its use. J. Dairy. Sci., 24: 905.

Salisbury, GW, Van Demark, NL (1961). Physiology of reproduction and artificial insemination of cattle. WH Freeman (Ed.), San Francisco. Pp: 415-425.

Salisbury, G.W., Van Demark, N.L. and Lodge, J.R. (1985). Physiology of Reproduction and Artificial insemination of cattle. $2^{\text {nd }}$ Edn. CBS Publishers and Distributers, New Delhi.

Saltman , P. (1989). Semen. Hematol., 26 : 249-256.

Salvader, I.,J.Yantz,M.P.Viudes-De-Castro,E.A.Gomez and M.A.Silvestre.(2006). Effect of solid storage on caprine semen conservation at $50^{\circ} \mathrm{C}$. Theriogenology, 64:252-260.

Sanctez- Partida, L.G., Setchell, B.P. and Maxiwell, W.M.C. (1997). Epididymal compounds and anti oxidants in diluents for the frozen storage of ram spermatozoa. Reprod.Fertil.Dev., 9:689-699.

Sarma, P.V. (1995). A simplified staining technique for evaluation of acrosomal status of sperm cells. Indian J. Anim. Reprod., 16:127 - 128.

Saroof, J. and Mixner, J.P. (1955). The relationship of egg yolk and glycerol content of diluters and glycerol equilibration time to survival of bull spermatozoa after low temperature freezing. J. Dairy Sci., 3:292.

Satish Kumar and Kadirvel G. (2007). Functional assessment of frozen thawed buffalo spermatozoa using cervical mucus penetration test. XXIII Annual convention of ISSAR and national symposium .Bhubaneswar. pp: 234.

Satish Kumar (2007). Advances in semen preservation: concept and its minimization. XXIII Annual convention of ISSAR and national symposium .Bhubaneswar. pp: 207.

Saxena, M.S. (2000). Veterinary Andrology and artificial insemination, $1^{\text {st }}$ Edn., CBS publishers and distributors, New Delhi, India. 
Saxena, V.B. and Tripathy, S.S. (1978). Studies on the physico-chemical attributes and preservability of semen of CB bulls. Indian J. Anim. Sci., 48: 865-869.

Schluter, U. (1975). Evaluation of data on the collection, examination and preservation of bull semen with reference to biological and economic aspects. Thesis, Tierartzatliche Hochschule. Hannover G.F.R. (Anim. Breed. Abstr., 47:3625).

Stachecki, J.J., Ginsburg, K.A., Leach, R.E. and Larmant, D.R. (1993). Computer-assisted semen analysis (CASA) of epididymal sperm from the domestic cat. J. Androl., 14:60-65

Seit, B. (1953). Provisional investigations on diluters for bull semen useable in Indonesia. Hemera Zoa., 60:19 (Anim. Breed. Abstr., 22:155).

Selvaraju, S., Ravindra, J.P., Ghosh, J., Gupta, P.S.P and Suresh, K.P. (2008). Evaluation of sperm functional attributes in relation to in vitro sperm-zona pellucida binding ability and cleavage rate in assessing frozen thawed buffalo (Bubalus bubalis) semen quality. Anim Reprod Sci., 106(3-4):311-21

Selvaraju, S., Ghosh, J. and Ravindra, J.P. (2008). Prognostic value of various spermatological attributes as predictors of zona binding and zona penetration of buffalo (Bubalus bubalis) semen. Reprod. Dome. Anim, 44(1):6-11

Shannon, P. and Vishwanath, R. (1995). The effect of optimal and suboptimal concentrations of sperm on the fertility of fresh and frozen bovine semen and a theoretical model to explain the fertility differences. Anim. Reprod. Sci., 39: 1-10.

Shannon, P. and Curson, B. (1972). Toxic effect and action of dead sperm on diluted bovine semen. J. Dairy Sci., 55: 614-620.

Sharma, M.L., Mohan G. and Sahni K.L. (1980). A comparative study of acrosomal morphology of crossbred and Holstein- Friesian bull semen. Ind.J.Anim.Rrprod., 11(2) :96-99.

Sharma, R.S., Gaur, K.K., Pal, P.C., Monika Manocha, Deepak Tomer, Khan, A.A., Vinita, T., Vineetha, C. and Kriplani, A. (2005). Semen characteristics: Advancement in andrological assessment.Ind.J.Clinical Bio., 20(1):173-183.

Sharma, R.K. and Agarwal, A.(1996). Reactive oxygen species and male infertility. Urology, 48: $835-850$. 
Singh, J., Pangawkar G.R., Bisaws R.R. and Naresh Kumar.(1991). Studies on buffalo sperm morphology during various stages of freezing in certain extenders. Indian J. Anim.Reprod., 12(2):126-129.

Singh, K.H.M., Singh M.P. and Sinha A.K. (2007). Release of trasaminase subsequent to preservation in diluents containing EDTA and cysteine $\mathrm{Hcl}$ of Betal and crosses bred (Beetal X Black Bengal) buck spermatozoza. India J. Anim. Reprod., 28(1): 62-65.

Singh, K.T., Mohanty, B.N., Ray, S.K.H., Mohanty, D.N. and Pattanayak, G.R. (1993) Effect of spermatozoa concentration on freezability of bull semen. Indian J. Anim. Reprod., 14: 103104.

Singh, S.P., Pandit, R.K. and Bhadoria, H.B.S. (1997) Effect of freezing on seminal characteristics of Jersey, Sahiwal and their half bred bulls. Indian J. Anim. Reprod., 18 : 73-74.

Singh, L.P. and Purbey L.N. (1996). Effect of ultra low temperature on acrosomal integrity of buck spermatozoa in tris and citrate dilutors. Ind. J. Anim. Rrprod., 17 (1): 45-49

Sinha, M.P., A.K.Sinha, Sinka, B.K. and Prasad, P.I. (1996). The effect of Glutathione on motility, enzyme leakage and fertility of frozen goat semen. Theriogenology, 41: 237-243.

Słowińska, M., Karol, H., Ciereszko, A. (2008). Comet assay of fresh and cryopreserved bull spermatozoa. Cryobiology, 56(1):100-102.

Snedecor, G.W. and Cochran, W.G. (1967). Statistical methods. $6^{\text {th }}$ Edn. The Iowa State University Press, Ames, Iowa, USA.

Soderquist, L., Rodriguez-Martinez, H. and Janson, L. (1991). Post-thaw motility, ATP content and cytochrome $\mathrm{C}$ oxidase activity of AI bull spermatozoa in relation to fertility. Zentralbl Veterinarmed A., 38: 165-174.

Soderquist, L., Janson, L., Larsson, K. and Einarsson, S. (1991). Sperm morphology and fertility in AI bulls. J. Vet. Med. A., 38:534-543.

Sreejith, J., Nair, A.S., Brar, C.S., Ahuja, S.P., Sangha, S. and Chaudhary, K.C. (2005). A comparative study on lipid peroxidation, activities of antioxidant enzymes and viability of cattle and buffalo bull spermatozoa during storage at refrigeration temperature. Anim. Reprod. Sci., 96: 21-29. 
Stalhammar, E.M., Janson, L. and Philipsson, J. (1994). The impact of sperm motility on nonreturn rate in preselected dairy bulls. Reprod. Nutr. Dev., 34: 37-45.

Steinbach, J. and Foote, R.H. (1967). Osmotic pressure and $\mathrm{P}^{\mathrm{H}}$ effects on survival of frozen bovine spermatozoa. J. Dairy Sci., 50: 205.

Steven Verberckmoes. (2004). Preservation of fresh bovine semen and utero-tubal junction insemination in cattle. $\mathrm{PhD}$ thesis Submitted to Universiteit Gent, 2004.

Stradaioli, G., Noro, T., Sylla, L. and Monaci, M. (2007). Decrease in glutathione (GSH) content in bovine sperm after cryopreservation: comparison between two extenders. Theriogenology, 67(7):1249-55.

Suarez, S.S. and Dai, X. (1992). Hyper activation enhances mouse sperm capacity for penetrating visco-elastic media. Biol. Reprod., 46: 686-691.

Suarez, S.S., Dai, X.B., DeMott, R.P., Redfern, K. and Mirardo, M.A. (1992). Movement characteristics of boar sperm obtained from the oviduct or hyper activated in vitro. J. Androl., 13: 75-80.

Sukardi, S., Curry, M.R. and Watson, P.F. (1997). Simultaneous detection of the acrosomal status and viability of incubated ram spermatozoa using fluorescent markers. Anim. Reprod. Sci., 46:89-96.

Suleiman, S.A., Ali, M.E., Zaki, M.S., Malik, E.M.E.A. and Nast, M.A. (1996). Lipid peroxidation and human sperm motility: protective role of vitamin E. J. Androl., 17(5): $530-537$.

Swanson, E.W. and Herman, H.A. (1944). The correlation between some characteristics of dairy bull semen and conception rate. J. Dairy. Sci., 27: 297-302.

Szczesniak-Fabianczyk, B., M. Bochenek, Z.Smorag and M.A. Silvertre. (2006). Effect of antioxidants added to boar semen extender on the semen survival time and sperm chromatin structure. Reprod. Biol., 3: 81-87.

Tang, S., Garrett, C. and Gordon Baker, H.W. (1999). Comparison of human cervical mucus and artificial sperm penetration media. Hum. Reprod., 14: 2812-2817.

Tasseron, F.A. and Schindler, H. (1977). Acrosome damage of sperm of ram spermatozoa durind dilution, cooling and freezing. J.Reprod. Fert., 51:461-462. 
Tesarik, M.D., Mendoza, C. and Carreras A. (1993). Fast acrosome reaction measure: a highly sensitive method for evaluating stimulus-induced acrosome reaction. Fertil. Steril., 59:424-430.

Thomas, C.A., Garner, D.L., DeJarnette, J. M. and Marshall, CE. (1998). Effect of cryopreservation on bovine sperm organelle function and viability as determined by flow cytometry. Biol Reprod.,58: 786-93.

Tripathy, V.N. and Prabhu, S.S. (1968). Variation in semen quality, reaction time and conception rate in Hariana bull due to season of the year. J. Anim. Morph. Physiol., 15:127-134.

Tuli, R.K. Batabyal, A.K., Singal, S.P. and Razdan, M.N. (1988). Seminal characteristics and deep freezing of CB bull semen using Tris yolk glycerol extender. Indian J.Anim.Reprod., 9: $16-18$.

Uysal, O. and Korkmaz, T. (2004). Evaluation of membrane integrity by hypo-osmotic swelling test in canine spermatozoa. Ind. Vet. J., 21:1229-1231.

Uysal O., Korkmaz, T. and Tosun, H. (2005). Effect of bovine serum albumin on freezing of canine semen. Ind.Vet.J., 82:97-98.

Uysal, O. and Bucak, M.N. (2007). Effects of Oxidized Glutathione, Bovine Serum Albumin, Cysteine and Lycopene on the Quality of Frozen-Thawed Ram Semen. Acta Vet. Brno., 76: $383-390$.

Uysal, O., Buck, M.N., Yavas, I. and Varisli.O. (2007).Effect of various antioxidants on the Quality of frozen thawed bull semen.J.Anim.Vet.Ad., 6(12):1362-1366.

Van Demark, N.L., Salisbury, G.W., and Bratton, R.W. (1949) Oxygen damage to bull spermatozoa and its prevention by catalase. J. Dairy. Sci., 32: 353-360.

Vilfan, I.D., Conwell, C.C. and Hud, N.V. (2004). Formation of native-like mammalian sperm cell chromatin with folded bull protamine. J. Biol. Chem., 279(19): 20088-95.

Vizcarra, J.A. and Ford, J.J. (2006). Validation of the sperm mobility assay in boars and stallions. Theriogenology, 66:1091-1097. 
Wainer, R., Merlet, F., Bailly, M., Lombroso, R., Camus, E. and Bisson, JP. (1996). Prognostic sperm factors in intra-uterine insemination with partner's sperm. Contracept. Fertil Sex, 24:897-903.

Wang, X., Sharma, R.K., Gupta, A., George, V., Thomas, A.J., Falcone, T. and Agarwal, A. (2003). Alterations in mitochondria membrane potential and oxidative stress in infertile men a prospective observational study. Fertil. Steril., 80 (2): 844-850.

Watson, P.F. (1975).Use of Giemsa stain to detect change in acrosome of frozen ram spermasatozoa.Vet.Rec., 97: 12-15.

Watson, P.F. (1995). Recent development and concepts in cryo preservation of spermatozoa and assessment of their post thawing function. Reprod. Fertil. Dev., 7:871-891.

Watson, P.F. (2000). The causes of reduced fertility with preserved semen. Anim. Reprod. Sci., 60: $481-492$.

Williams, WW. (1920). Technique of collecting semen for laboratory examination with a view of several diseased bulls. Cornell Veterinarian, 10: 87-89.

Wolf, E. (1996). Biochemical and endocrinological parameters as indicators of freezability of semen of bulls of different ages. Thesis, Tierarztliche Hochshule, Hannover, pp.78 (Anim. Breed. Abstr., 64:7305).

Wood, P.D.P., Foulkes, J.A., Shaw, R.C. and Melrose, D.R. (1986). Semen assessment, fertility and the selection of Hereford bulls for use in AI. J. Reprod. Fertil. 76: 783-795.

Yanagimachi R. Mammalian fertilization. In: Knobil E, Neill J (eds). (1994). Physiology of Reproduction. Raven press; New York: 189-317.

Yang, SE. and Chou, CC. (2000). Growth and survival of Escherichia Coli O157:H7 and Listeria monocytogenes in egg products held at different temperatures. J. Food. Prot., 63: 907911.

Yavuz Öztürkler. (2001). Comparison of ovine spermatozoal morphological features after staining of fixation and assessment of morphological abnormalities in dead/live spermatozoa. Turk. J. Vet. Anim. Sci., 25 : 675-680. 
Zakariya Nur, Ibrahim Dogan, Ulgen Gunay and Kemal Soylu, M. (2005). Relationship between sperm membrane integrity and other semen quality charactertes of semen of sannen goat bucks.Bull Vet.Inst Pulaway, 49:183-187.

Zekariya Nur and Kemal Soylu. M. (2003). The comparison of plasma membrane integrity with morphological characteristics of frozen bull semen. Uludag Uni.J.Fac.Vet.Med., 22(1-23):51-53.

Zemjanis, R. (1970). Diagnostic and therapeutic technique in animal reproduction. $2^{\text {nd }}$ Edn. The Williams and Wilkine, Company, Baltimore. 